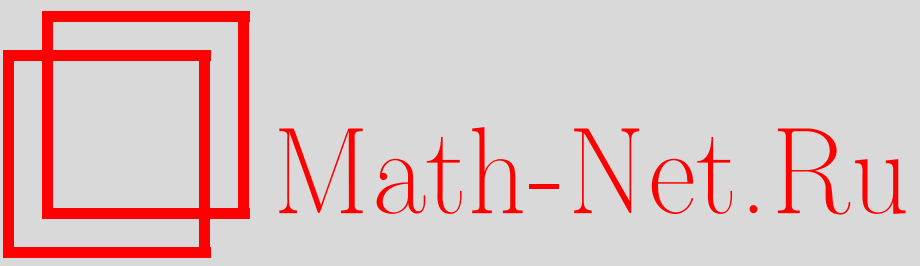

Л. Д. Пустыльников, Бесконечномерные нелинейные обыкновенные дифференциальные уравнения и теория КАМ, УМН, 1997, том 52, выпуск 3, 105-160

DOI: https://doi.org/10.4213/rm850

Использование Общероссийского математического портала Math-Net.Ru подразумевает, что вы прочитали и согласны с пользовательским соглашением

http://www.mathnet.ru/rus/agreement

Параметры загрузки:

IP: 54.84 .234 .179

26 апреля 2023 г., 13:24:13 


\title{
БЕСКОНЕЧНОМЕРНЫЕ НЕЛИНЕЙНЫЕ ОБЫКНОВЕННЫЕ ДИФФЕРЕНЦИАЛЬНЫЕ УРАВНЕНИЯ И ТЕОРИЯ КАМ
}

\author{
Л. Д. Пустыльников
}

\section{СОДЕРЖАНИЕ}

Введение

Обшие обозначения иогределения .

Глава 1. Теория уравнения с потенциалом Френкеля-Конторовой . . . . . . . . . . . 112

$\S 1$. Неоднозначная разрешимость и неразрешимость задачи Коши для стандарт-

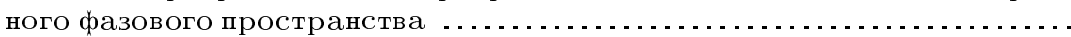

$\S 2$. Введение фазовых пространств и однозначная разрешимость уравнения в классах бесконечно-дифференцируемых и аналитических функций ....

$\S 3$. Построение семейств периодических решений, решений, имеющих тип бегу-

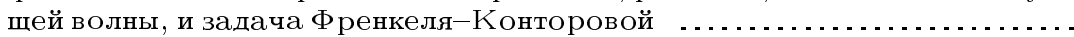

$\S 4$. Неустойчивость стационарных и периодических решений в случае равных

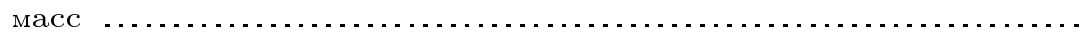

$\S 5$. Устойчивость стационарных решений и бесконечно-мерный аналог теории

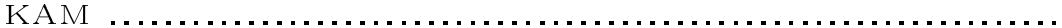

§6. Доказательство теоремы $1.10 \ldots \ldots$

$\S 7$. Гамильтонова форма уравнения с потенциалом Френкеля-Конторовой $\ldots . . .134$

$\S 8$. Лагранжева форма уравнения с потенциалом Френкеля-Конторовой $\ldots . . . .137$

Глава 2. Теория уравнения с нелинейностью Ферми-Паста-Улама . . . . . . . . . 138

$\S 1$. Построение семейств периодических решений и решений, порождаюших ко-

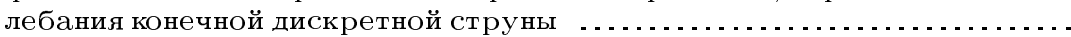

$\S 2$. Введение фазовых пространств и однозначная разрешимость уравнения

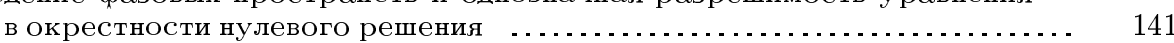

§3. Устойчивость нулевого решения и бесконечно-мерный аналог теории КАМ .144

$\S 4$. Доказательство теоремы 2.4 . . . . . . . . . . . . . . . . . . . . . . . . . . . 146

$\S 5$. Об устойчивости относительно неавтономной динамической системы с дискретным временем $\ldots \ldots \ldots \ldots \ldots \ldots \ldots \ldots \ldots \ldots \ldots \ldots \ldots \ldots \ldots \ldots \ldots \ldots$

$\S 6$. Локальная неоднозначная разрешимость и неразрешимость уравнения для стандартного фазового пространства . . . . . . . . . . . . . . . . . .

Глава 3. Бесконечно-мерные обыкновенные дифференциальные уравнения об-

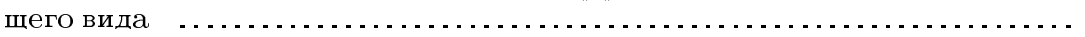

$\S 1$. Формальная однозначная разрешимость и единственность аналитических

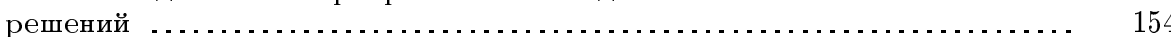

$\S 2$. Устойчивость стационарных решений . . . . . . . . . . . . . . . . . . . . . . . . 157

Список литературы ......................................... 158

(C) л. Д. Пустыльников 1997 


\section{Введение}

Эта работа посвящена исследованию двух классов бесконечномерных систем Гамильтона, которые определяются соответственно следуюшими системами обыкновенных дифференциальных уравнений Ньютона:

$$
\begin{aligned}
m_{n} \frac{d^{2} q_{n}}{d t^{2}}= & \varphi\left(q_{n}\right)+q_{n+1}-2 q_{n}+q_{n-1}, \quad n \in \mathbb{Z}, \\
m_{n} \frac{d^{2} x_{n}}{d t^{2}}= & x_{n+1}-(2-c) x_{n}+x_{n-1} \\
& +\beta\left[\left(x_{n+1}-x_{n}\right)^{r}-\left(x_{n}-x_{n-1}\right)^{r}\right], \quad n \in \mathbb{Z}
\end{aligned}
$$

(и описьвают движение счетного числа массивных точек $P_{n}$, занумерованных всеми целыми числами $n$ ), а также возмушениями этих уравнений

$$
\begin{aligned}
m_{n} \frac{d^{2} q_{n}}{d t^{2}}= & \varphi\left(q_{n}\right)+q_{n+1}-2 q_{n}+q_{n-1}+\psi_{n}(\vec{q}), \quad n \in \mathbb{Z} \\
m_{n} \frac{d^{2} x_{n}}{d t^{2}}= & x_{n+1}-(2-c) x_{n}+x_{n-1} \\
& +\beta\left[\left(x_{n+1}-x_{n}\right)^{r}-\left(x_{n}-x_{n-1}\right)^{r}\right]+\psi_{n}(\vec{x}), \quad n \in \mathbb{Z}
\end{aligned}
$$

с помощью функций $\psi_{n}(\vec{q}), \psi_{n}(\vec{x})$ общего вида.

В указанных уравнениях (0.1)-(0.4) $m_{n}$ - положительньй параметр, характеризующий массу точки $P_{n}, \varphi(q)$ - вешественная, бесконечно-дифференцируемая функция, имеющая по $q$ период $a \neq 0, r$ - нечетное натуральное число, $r \geqslant 3, c$ и $\beta$ - константы, $c \geqslant 0, \beta>0, q_{n}$ и $x_{n}$ - зависимые переменные, $\vec{q}=\left(q_{n}\right)$ и $\vec{x}=\left(x_{n}\right)$ - бесконечно-мерные векторы, составленные из координат $q_{n}$ и $x_{n}(n \in \mathbb{Z})$ соответственно, $t$ - независимая переменная, характеризующая время. Уравнение (0.1) есть система с потенциалом Френкеля-Конторовой [1], а уравнение (0.2) представляет собой обобщение уравнения Ферми-Паста-Улама [2]-[4].

В настоящей работе построена полная теория уравнений (0.1)-(0.4), включающая правильные определения фазовых пространств, решение задачи Коши о сушествовании и единственности решений в классах бесконечно-дифференцируемых и аналитических по $t$ функций, построение семейств периодических решений и решений типа бегущей волны, построение бесконечно-мерных аналогов теории Колмогорова-Арнольда-Мозера (теории КАМ) и семейств квази-периодических решений с континуальным, счетным и любым конечным числом рационально-независимых частот и доказательства устойчивости стационарных решений в бесконечно-мерных фазовых пространствах. Последнее утверждение обнаруживает принципиально новое явление, касающееся устойчивости в неинтегрируемых гамильтоновых системах с числом степеней свободы большим, чем 2, в связи с тем, что в этом случае инвариантные торы не разделяют фазового пространства. Согласно общим представлениям [7]-[11], при увеличении размерности системы возможностей для неустойчивого поведения становится все больше, и поэтому в бесконечно-мерном случае такая неустойчивость безусловно должна иметь место для систем общего вида. Однако,

Работа вьполнена при частичной поддержке Российского фонда фундаментальных исследований (грант № 96-01-01411). 
как следует из настоящей работы, для важных классов бесконечно-мерных гамильтоновых систем (гл. 1,2 ) стационарные решения являются устойчивьми в смысле Ляпунова в бесконечно-мерном фазовом пространстве (теорема 1.10, следствие 1.4 , гл. 1; теорема 2.4, следствие 2.3, гл. 2) и подобные, но более слабые, утверждения об устойчивости справедливы для близких к ним уравнений (теоремы 3.4, 3.5; замечания $3.4,3.5$; гл. 3). Теоремы $1.10,2.4$ и их следствия 1.4, 2.3 показьвают, что малые изменения начальных данных в соответствии с нормами в фазовых пространствах уравнений в окрестности стационарных решений приводят к малым изменениям всех координат и импульсов решений соответствующих гамильтоновых систем во все моменты времени. При этом в окрестностях рассматриваемых сташионарных решений имеет место усиленный вариант теории KAM: сушествование семейств не только конечно-мерных инвариантных торов [5]-[22], но также семейств инвариантных торов, которые имеют счетную и даже континуальную размерность; более точно, построены многообразия, которые образуют существенные подмножества тора континуальной размерности (определение существенного подмножества дано в п. 13) следуюшего раздела). Решения уравнений порождают на этих торах квази-периодические движения (теорема 1.11, следствие 1.5, гл. 1; теорема 2.5, следствие 2.4, гл. 2). Применительно к бесконечно-мерным неинтегрируемым гамильтоновым системам эти результаты являются наиболее сильные, так как в вариантах теории КАМ нелинейных уравнений, разработанных к настоящему времени были построены квази-периодические решения только с конечньп ([29]) или счетным ([24]-[28]) множеством частот. Отметим, что задача изучения поведения решений на множестве начальных данных, являюшемся дополнением как к конечно-мерным, так и к счетно-мерным инвариантным торам, ставилась во многих работах (см., например, [7]-[11], [19], [23], [24]).

Важньм стимулом для построения периодических и квази-периодических решений бесконечно-мерных уравнений типа (0.1)-(0.2) является так назьваемая проблема Ферми-Паста-Улама ([2]-[4]). Эти ученые численно исследовали систему, состоящую из большого числа уравнений $(0.2)$, в которых все константы $m_{n}=1$, константа $c=0$, а число $r=3$, и, вопреки ожиданиям, обнаружили решения, которые имеют устойчивое поведение, похожее на почти-периодическое. Обшим моментом в попытках обоснования этих результатов является замена системы обыкновенных дифференциальных уравнений (0.2) уравнением в частных производных [54]. В нашей же работе изучается система, состояшая из бесконечного числа уравнений (0.2) с нелинейньм потенциалом Ферми-Паста-Улама или его обобщением $(c \geqslant 0, r \geqslant 3)$, как таковая (гл. 2). Результаты, полученные здесь, состоят в следуюшем: если константа $c=0$, а все массы $m_{n}$ равны между собой, то сушествует и явно строится семейство периодических решений (теорема 2.1, предложение 2.1, гл. 2), а если при всех целых $n m_{n} \leqslant M$ и константа $c$ удовлетворяет условиям $0<c<4, c \neq 2-2 \cos \frac{2 \pi s}{d}$, где $s=0, \pm 1, \ldots, \pm d$, $d=1, \ldots, r+1$, то существуют такие константы $m_{n}^{0}>0(n \in \mathbb{Z})$, что если при каком-либо $n_{0} \geqslant 0$ и $|n| \geqslant n_{0}$ массы $m_{n}$ удовлетворяют неравенствам $m_{n} \leqslant m_{n}^{0}$, то нулевое решение $x_{n}=0(n \in \mathbb{Z})$ уравнения $(0.2)$ устойчиво по Ляпунову и в любой его окрестности имеет место бесконечно-мерньй аналог теории КАM (теоремы 2.4, 2.5). Отметим, что условия теорем 2.4 и 2.5 будут вьполнены, если при сколь угодно большом числе $n_{0}$ для чисел $n$, удовлетворяюших неравенству $|n|<n_{0}$, выбрать массы 
$m_{n}$ равными между собой, а число $c$ выбрать сколь угодно близким к нулю, и в этом случае условия теорем 2.4 и 2.5 можно сколь угодно сильно приблизить к условиям эксперимента Ферми-Паста-Улама.

Другим весьма популярным в настоящее время объектом исследования, которому посвящена гл. 1 данной работы, является уравнение (0.1) с потенциалом Френкеля-Конторовой, введенное в работе [1] в связи с теорией дислокации в физике твердого тела. По сути своей уравнение (0.1) описьвает основополагаюшую систему классической механики, представляющую собой бесконечную цепочку обычных маятников, последовательно соединенных друг с другом. Для частного случая уравнения (0.1), при котором все массы $m_{n}$ равны между собой, а функция $\varphi(q)=-h \sin \frac{2 \pi q}{a}(h-$ положительная константа, не зависящая от $q$ ) в работе [1] была поставлена задача о нахождении решений, имеющих тип бегущей волны, т.е. решений $q_{n}(t)$, удовлетворяюших при всех вешественных $t$ и целых $n$ равенству $q_{n}(t)=q_{n+1}(t+\tau)$, где $\tau$ - положительная константа, не зависяшая от $n$ и $t$. В этой же работе [1] указанная задача решалась приближенно с помошью замены уравнения (0.1) уравнением в частных производных, и никакого строгого решения задачи на этом пути не получено до сих пор. В настоящей работе в $\S 3$ гл. 1 дано строгое решение задачи $\Phi$ ренкеля-Конторовой как для функции $\varphi(q)=-h \sin \frac{2 \pi q}{a}$, так и для периодической функции $\varphi(q)$ общего вида, причем в первом случае это решение имеет более конкретный характер, соответствуюший постановке задачи в [1].

Уравнение (0.1) имеет бесконечно много стационарных решений, не зависяших от времени $t$ и от масс $m_{n}$, которые в последнее время интенсивно изучались в связи с теорией Обри-Мазера о конфигурациях с наименьшей энергией [30]-[35]. Простейшие из них - это решения, имеюшие вид

$$
q_{n}^{0}(t) \equiv \eta+n a,
$$

где $\eta$ - такое значение, при котором $\varphi(\eta)=0$. Исследование поведения уравнения $(0.1)$ в окрестности стационарных решений приводит к задаче об их устойчивости.

Если массы $m_{n}$ всех частиц равны между собой, то, как показано в $\S 4$, стационарные решения вида (0.5), а также периодические решения, найденные в 33 (гл. 1), - неустойчивы. Такая неустойчивость имеет место, даже если массы $m_{n}$ равны нулю [36], так как в этом случае отображение $\left(q_{n-1}, q_{n}\right) \rightarrow\left(q_{n}, q_{n+1}\right)$ в окрестности решения $q_{n}^{0}(n \in \mathbb{Z})$ становится гиперболическим, и это обстоятельство имеет важное значение в теории фазовых переходов в модели Френкеля-Конторовой, а подобные неустойчивые траектории определяют основные состояния [36]-[40].

В случае же, когда массы $m_{n}$ при $|n| \rightarrow \infty$ достаточно быстро убывают, в $\S \S 5,6$ доказано, что для широкого класса функций $\varphi(q)$ стационарное решение $(0.5)$ в естественном смысле устойчиво по Ляпунову в бесконечно-мерных фазовых пространствах, а в любой его окрестности имеет место бесконечно-мерный аналог теории КАМ. В силу теоремы 1.10 и следствия $1.4(\S 5)$ результаты об устойчивости касаются гамильтоновой формы уравнения (0.1), и поэтому в $\S \S 7,8$ гл. 1 дается введение в гамильтонов и лагранжев формализм применительно к уравнению с потенциалом $\Phi$ ренкеля-Конторовой и приведены некоторые обшие теоремы, обобшаюшие классические теоремы конечно-мерной гамильтоновой и лагранжевой механики. 
Все результаты справедливы для функции $\varphi(q)=-h \sin \frac{2 \pi q}{a}$ и для всех близких к ней функций (следствия $1.1,1.2, \S 5)$. Отметим, что в работе [50] получены обобщения указанных вьше результатов на случай цепочек произвольной размерности, обобщаюших одномерную модель Френкеля-Конторовой и описьвающих движения атомов в многомерном твердом теле.

Большое внимание в настояшей работе уделяется проблеме однозначной разрешимости уравнений (0.1)-(0.4), т.е. задаче о правильной постановке задачи Коши о сушествовании и единственности решений. Это связано с тем обстоятельством, что, как неожиданно выяснилось, если использовать стандартное фазовое пространство, в котором начальные данные задаются значениями всех координат и их первых производных в начальный момент времени, то решения уравнений $(0.1)$ и $(0.2)$ в классе бесконечно-дифференцируемых по $t$ функций определяются неоднозначно, а в классе аналитических по $t$ функций вообще могут не сушествовать, причем для уравнения (0.1) эти явления имеют место как в системе без граничных условий, так и с граничньми условиями вида

$$
\lim _{n \rightarrow-\infty} q_{n}(t)=-\infty, \quad \lim _{n \rightarrow+\infty} q_{n}(t)=+\infty
$$

(теоремы 1.1, 1.2, §1, гл. 1; теорема 2.7, §6, гл. 2).

Указанные результаты о неоднозначной разрешимости и неразрешимости уравнений $(0.1)$ и (0.2) в стандартной постановке являются следствиями теорем 1.10 и 2.4 об устойчивости стационарных решений. В $\S 2$ гл. 1 и в $\S 2$ гл. 2 работы для классов бесконечно-дифференцируемых и аналитических функций построены фазовые пространства, в которых решения уравнений (0.1) и (0.2) сушествуют и единственны. Подобные же результаты, касающиеся аналитических функций, получены в 1 гл. 3 для обших уравнений $(0.3)$ и $(0.4)$, в которых функции $\psi_{n}(\vec{q}), \psi_{n}(\vec{x})$, принадлежат классу $W$ (определение $3.1, \S 1$, гл. 3 ), состоящему из аналитических функций, зависящих только от конечного числа координат $q_{n}$ и $x_{n}$ соответственно, причем сами эти координаты и их число могут быть разными у функций $\psi_{n}(\vec{q}), \psi_{n}(\vec{x})$ при разных $n$.

Результаты этой работы об устойчивости решений уравнений (0.1)-(0.4) сушественно используют теорему об устойчивости относительно неавтономной динамической системы с дискретным временем, зависяшей от параметра, которая сформулирована в $\S 5$ гл. 2 (теорема 2.6). При фиксированном значении параметра в случае, когда в нормальной форме Биркгофа предельного преобразования (равенство (2.55), §5, гл. 2) коэффищиент $\omega_{1}$ в линейном члене отличен от нуля, эта теорема является частным случаем теоремы 2 из [44] (ч. 2 , гл. V, 1 ). В том виде, как она сформулирована в $\S 5$ гл. 2, ее доказательство проводится так же, как доказательство теоремы 1 из работы [45] (§2). Отметим, что указанная теорема 2.6 и ее обобщения [44], [45] имеют много других применений. Они, в частности, позволяют получать результаты об устойчивости по Ляпунову траекторий в пятимерной системе Гамильтона (две степени свободы и время) обшего вида [46], которые существенным образом опираются на работу [47].

В связи с результатами, полученными в данной работе, здесь сфформулированы некоторые нерешенные проблемы. К ним относятся задачи об описании множества решений типа бегущей волны для уравнения $(0.1)$ (§ 3, гл. 1), множества периодических решений и решений, порождаюших колебания конечной дискретной струны для уравнения $(0.2)(\S 1$, гл. 2$)$ и гипотеза об описании множеств начальных данных, для которых 
сушествуют аналитические решения уравнений $(0.3)$ и $(0.4)(\S 1$, гл. 3$)$. В заключение отметим, что методы, применяемые в этой работе, могут быть использованы также при изучении таких явлений, как бесконечно-мерный хаос и бифуркации в динамических системах с бесконечным числом степеней свободы [48]. Основные результаты работы опубликованы в [41]-[43], [55]-[57] и докладьвались на заседании Московского математического общества 25 октября 1994 года и на симпозиуме в центре Банаха по эргодической теории и динамическим системам летом 1995 года [49].

Сразу после введения приведен список обших обозначений и определений, используемых в работе, который, в частности, содержит определения квази-периодических функций, решений и движений с произвольным множеством частот, а также определения торов любой размерности и существенного подмножества тора континуальной размерности. Кроме того, в отдельных главах и параграфах имеются другие обозначения и определения, ссылки на которые даются с указанием номеров определения, параграфа и главы.

\section{Общие обозначения и определения}

1) $\mathbb{Z}$ - множество целых чисел.

2) $\mathbb{R}$ - множество всех действительных чисел.

3) Аналитическая функция - функция, задаваемая абсолютно сходяшимся рядом.

4) Решение уравнений (0.1) и (0.3) обозначается символом $\left(q_{n}(t)\right)$, а решение уравнений $(0.2)$ и $(0.4)$ обозначается символом $\left(x_{n}(t)\right)$.

$5)$ Решение $\left(q_{n}(t)\right)$ уравнений $(0.1),(0.3)$ и решение $\left(x_{n}(t)\right)$ уравнений $(0.2),(0.4)$ называются бесконечно-дифференцируемыми, если при всех $n \in \mathbb{Z}$ функции $q_{n}(t), x_{n}(t)$ яляются бесконечно-дифференцируемыми на прямой $t \in \mathbb{R}$.

6) Решение $\left(q_{n}(t)\right)$ уравнений $(0.1),(0.3)$ и решение $\left(x_{n}(t)\right)$ уравнений $(0.2),(0.4)$ называются аналитическими, если при всех $n \in \mathbb{Z}$ функции $q_{n}(t), x_{n}(t)$ являются целыми аналитическими функциями на прямой $t \in \mathbb{R}$.

7) Определение квази-периодических функций с конечным или счетньм количеством частот.

Рассмотрим комплексно-значную функцию $F=F\left(\left(y_{\nu}\right)\right)$ от некоторого множества независимых вещественных переменных $y_{\nu} \in \mathbb{R}$, количество которых может быть конечным или счетным; т.е. параметр $\nu$, нумерующий переменные $y_{\nu}$, принадлежит множеству $B$, мощность которого конечна или счетна [53]. Предположим, что $F$ имеет по каждой переменной $y_{\nu}$ период $T>0$ и при фиксации всех переменных $y_{\nu}$, кроме одной переменной $y_{\nu_{0}}$, и подстановке этого набора (фиксированных переменных $y_{\nu}$ для $\nu \neq \nu_{0}$ и нефиксированной переменной $y_{\nu_{0}}$ ) в качестве аргументов $F$ мы получаем аналитическую функцию относительно $y_{\nu_{0}}$. Предположим, далее, что имеется множество вешественных чисел $\omega_{\nu}$, занумерованных параметром $\nu \in B$. Тогда функция $G(t)=F\left(\left(\omega_{\nu} t\right)\right)$ от вешественного переменного $t$, полученная в результате подстановок в качестве аргументов $y_{\nu}$ функции $F$ их значений $y_{\nu}=\omega_{\nu} t$, назьвается квази-периодической функцией с частотами $\omega_{\nu}(\nu \in B)$, а их количество есть мощность множества $B$.

Характерный пример: $G(t)=\sum_{k} h_{k} e^{i\left(u_{k}+\omega_{k} t\right)}$, где $k$ пробегает конечное или счетное множество, $h_{k}, u_{k}$ - числа, $u_{k}$ - вещественное, $\sum_{k}\left|h_{k}\right|<\infty, i$ - мнимая единица.

8) Определение пространства квази-периодических функций с континуальным множеством частот. 
Пусть $\mathscr{D}$ - произвольное ограниченное (содержащееся в некотором отрезке) множество на прямой $\mathbb{R}$, имеющее положительную лебегову меру, и рассмотрим множество $C_{\mathscr{D}}$ комплексно-значных функций $G(t)$ одного вещественного переменного $t$, имеюших вид

$$
G(t)=\int_{\omega \in \mathscr{D}} h(\omega) e^{i(u(\omega)+\omega t)} d \omega,
$$

где $h(\omega)$ - абсолютно интегрируемая функция, $u(\omega)$ - вешественная измеримая и всюду определенная функция на $\mathscr{D}$, а $i$ - мнимая единица. Пространство $Q_{\mathscr{D}}$ квази-периодических функций, у которых частоты принадлежат множеству $\mathscr{D}$, есть наименьшее линейное пространство комплексно-значных функций от переменного $t$, содержащее множество $C_{\mathscr{D}}$ и удовлетворяющее следующим свойствам:

а) вместе с любыми двумя функциями оно содержит их произведение;

б) если функции $G_{n}(t) \in Q, a_{n}$ - числа $(n=1,2, \ldots)$ и $\sum_{n=1}^{\infty}\left|a_{n} G_{n}(t)\right|<\infty$, то функция

$$
\sum_{n=1}^{\infty} a_{n} G_{n}(t) \in Q_{\mathscr{D}}
$$

(наименьшее пространство, - это перечисление всех пространств с указанньми свойствами).

9) Определение рационально-независимых частот.

Частоты $\omega \in \mathscr{D}$ называются рационально-независимьми, если любая конечная сумма $a_{1} \omega_{1}+\cdots+a_{s} \omega_{s}$, в которой $\omega_{k} \in \mathscr{D}, a_{k}$ - рациональные числа $(1 \leqslant k \leqslant s)$, обрашается в ноль тогда и только тогда, когда все $a_{k}=0$.

$10)$ Решение $\left(q_{n}(t)\right)$ уравнения $(0.1)$ называется квази-периодическим с частотами, принадлежашими множеству $\mathscr{D}$, если при всех $n \in \mathbb{Z} q_{n}(t)$ - квази-периодическая функция с частотами из $\mathscr{D}$.

$11)$ Решение $\left(x_{n}(t)\right)$ уравнения (0.2) назьвается квази-периодическим с частотами, принадлежащими множеству $\mathscr{D}$, если при всех $n \in \mathbb{Z} x_{n}(t)$ - квази-периодическая функция с частотами из $\mathscr{D}$.

12) Определение тора Tor $^{d}$ произвольной размерности.

Стандартная окружность $S^{1}=\{u: 0 \leqslant u<2 \pi\}=\mathbb{R} \backslash 2 \pi \mathbb{Z}$. Пусть символ $d$ обозначает мошность произвольного множества $\mathscr{D} \subset \mathbb{R}$. Topoм Tor $^{d}$ размерности $d$ назьвается множество, точка которого есть произвольная функция $u(\nu)=u_{\nu}$ на множестве $\mathscr{D}(\nu \in \mathscr{D})$, удовлетворяюшая неравенству $0 \leqslant u(\nu)<2 \pi$.

13) Определение существенного подмножества тора $\operatorname{Tor}^{d}$ континуальной размерности.

Рассмотрим тор $\operatorname{Tor}^{d}$, в котором символ $d$ обозначает мошность множества $\mathscr{D}$, которое представляет собой отрезок $\nu_{1} \leqslant \nu \leqslant \nu_{2}$ на прямой $\mathbb{R}$. Существенньг подмножеством тора $\operatorname{Tor}^{d}$ назьвается множество, состоящее из всех точек тора Tor ${ }^{d}$, для которых соответствующая функция $u(\nu)$ (см. п. 12)) измерима по Лебегу.

14) Определение квази-периодического движения на торе произвольной размерности.

Рассмотрим тор $\operatorname{Tor}^{d}$, в котором $d$ есть мошность множества $\mathscr{D}$, а произвольная точка, принадлежашая $\operatorname{Tor}^{d}$, обозначается через $u_{\nu}$. Пусть, далее, $\omega_{\nu}=\omega(\nu)-$ вещественно-значная функция на множестве $\mathscr{D}$. Квази-периодическим движением на тоpe $\operatorname{Tor}^{d}$ с частотами $\omega_{\nu}$ называется движение точки со временем $t$, которая в момент 
$t=0$ совпадает с точкой $u_{\nu}$, а в произвольньй момент времени $t$ совпадает с точкой $\widetilde{u}_{\nu}(t)=u_{\nu}+\omega_{\nu} t(\bmod 2 \pi)$.

15) Уравнение назьвается однозначно-разрешимьм в фазовом пространстве, если для любых начальных данных из этого пространства сушествует единственное решение.

16) Уравнение назьвается неразрешимым в фазовом пространстве, если существуют такие начальные данные из этого пространства, для которых решение не сушествует, и назьвается разрешимым в противоположном случае.

17) Уравнение называется неоднозначно разрешимьм в фазовом пространстве, если оно разрешимо и сушествуют такие начальные данные из этого пространства, для которых сушествует более одного решения.

18) Нумерация формул, теорем, лемм, предложений на протяжении всей статьи производится согласно следующему правилу: первая цифра обозначает номер главы, а затем идет номер соответствующего утверждения в этой главе.

\section{Глава 1.}

\section{Теория уравнения с потенциалом Френкеля-Конторовой}

Эта глава посвящена уравнению

$$
m_{n} \frac{d^{2} q_{n}}{d t^{2}}=\varphi\left(q_{n}\right)+q_{n+1}-2 q_{n}+q_{n-1}, \quad n \in \mathbb{Z}
$$

в котором $m_{n}>0, \varphi(q)$ - бесконечно-дифференцируемая функция, имеющая по $q$ период $a>0$, т.е. при всех $q$

$$
\varphi(q)=\varphi(q+a) .
$$

\section{$\S$ 1. Неоднозначная разрешимость и неразрешимость задачи Коши для стандартного фазового пространства}

Прежде чем определить фазовые пространства, в которых решение уравнения (1.1) существует и единственно (§2), мы докажем, что для стандартного (общепринятого) фазового пространства (определение 1.1) решение уравнения (1.1) в классе бесконечно-дифференцируемых функций $q_{n}(t)$ - не однозначно, а в классе аналитических функций $q_{n}(t)$ вообше может не существовать. При этом, когда мы изучаем уравнение (1.1) в классе аналитических функций, то всегда предполагается, что функция $\varphi(q)$ в (1.2) - аналитическая.

ОПРЕДЕЛЕНИЕ 1.1. Введем пространство $\Omega^{*}$ начальных данных для уравнения (1.1), точкой которого $\left\{\left(q_{n}^{*}\right),\left(v_{n}^{*}\right)\right\}$ являются две двусторонние последовательности чисел $\left(q_{n}^{*}\right),\left(v_{n}^{*}\right)$ такие, что при всех $n \in \mathbb{Z}$ вьполняются равенства $q_{n}^{*}=q_{n}\left(t^{*}\right)$, $v_{n}^{*}=\frac{d q_{n}}{d t}\left(t^{*}\right)$, где $t^{*}$ - начальньй момент времени.

В этом параграфе мы изучаем задачу об однозначной разрешимости уравнения (1.1) с фазовьм пространством $\Omega^{*}$ как без граничных условий (теорема 1.1 ), так и с граничными условиями (теорема 1.2) следуюшего вида:

$$
\lim _{n \rightarrow-\infty} q_{n}(t)=-\infty, \quad \lim _{n \rightarrow+\infty} q_{n}(t)=+\infty .
$$

Результаты этого исследования оказались отрицательными и формулируются в виде нижеследующих двух теорем. 
ТеОрема 1.1. Решение уравнения (1.1) в классе бесконечно-дифференцируемых nо $t$ функций $q_{n}(t)$ всегда существует при всех $t \in \mathbb{R}$, но не единственно, тогда как в классе аналитических по $t$ функиий $q_{n}(t)$ оно существует не всегда в сколь угодно малой окрестности значения $t^{*}$, но в тех случаях, когда существует, это решение - единственное.

ДокАЗАТЕльство. Если заданы начальные данные $\left\{\left(q_{n}^{*}\right),\left(v_{n}^{*}\right)\right\} \in \Omega^{*}$, то из уравнения (1.1) однозначно определяются значения вторых производных функций $q_{n}(t)$ в точке $t^{*}$, а продифференцировав уравнение (1.1) $k$ раз, мы определим значения $\frac{d^{2+k} q_{n}}{d t^{2+k}}\left(t^{*}\right)$ через значения $\frac{d^{s} q_{n}}{d t^{s}}\left(t^{*}\right)$ и $q_{n}\left(t^{*}\right)$ при всех $n \in \mathbb{Z}, 1 \leqslant s \leqslant k$. Таким образом, имея начальные данные, мы однозначно определим значения всех производных от всех функций $q_{n}(t)$ в точке $t^{*}$.

ЛЕмма 1.1. Для любого вещественного числа $t^{*}$ и любой наперед заданной последовательности вещественных чисел $a_{k}(k=0,1,2, \ldots)$ существует бесконечное множсество бесконечно-дифференцируемых функиий $q(t)$, определенных на прямой $t \in \mathbb{R}$ и таких, что $q\left(t^{*}\right)=a_{0}, \frac{d^{k} q}{d t^{k}}\left(t^{*}\right)=a_{k}(k=1,2, \ldots)$.

Применяя лемму 1.1, построим бесконечно-дифференцируемые функции $q_{0}(t)$ и $q_{1}(t)$, у которых их значения в точке $t^{*}$ совпадают соответственно с $q_{0}^{*}$ и $q_{1}^{*}$, а значения всех их производных в точке $t^{*}$ совпадают соответственно со значениями производных $\frac{d^{k} q_{0}}{d t^{k}}\left(t^{*}\right), \frac{d^{k} q_{1}}{d t^{k}}\left(t^{*}\right)(k=1,2, \ldots)$, найденньми выше по начальным данньм. Далее, при известных функциях $q_{0}(t), q_{1}(t)$ с помощью равенства (1.1) однозначно определяются функции $q_{n}(t)$ для всех $n \in \mathbb{Z}$, и при этом их значения и значения их первых производных в точке $t^{*}$ совпадают соответственно с начальными данньми $q_{n}^{*}, v_{n}^{*}$. Так как в силу леммы 1.1 функции $q_{0}(t), q_{1}(t)$ определены неоднозначно, то из предыдущего утверждения следует утверждение теоремы 1.1 о неоднозначной разрешимости уравнения (1.1) в классе бесконечно-дифференцируемых функций. Для доказательства той части теоремы 1.1, которая касается аналитических решений, заметим, что аналитические функции $q_{0}(t)$ и $q_{1}(t)$, которые имеют заданные значения и значения всех производных в точке $t^{*}$, либо не существуют, либо определены однозначно. Для того чтобы доказать несушествование аналитических решений уравнения (1.1), зададим две бесконечно-дифференцируемые функции $q_{0}(t)$ и $q_{1}(t)$ так, чтобы не существовали аналитические в любой окрестности точки $t^{*}$ функции $\widetilde{q}_{0}(t)$ и $\widetilde{q}_{1}(t)$, удовлетворяющие условиям

$$
q_{\nu}\left(t^{*}\right)=\widetilde{q}_{\nu}\left(t^{*}\right), \quad \frac{d^{k} q_{\nu}}{d t^{k}}\left(t^{*}\right)=\frac{d^{k} \widetilde{q}_{\nu}}{d t^{k}}\left(t^{*}\right) \quad(\nu=0,1 ; \quad k=1,2, \ldots)
$$

Зная функции $q_{0}(t)$ и $q_{1}(t)$, с помошью равенства $(1.1)$ находим функции $q_{n}(t)$, удовлетворяюшие (1.1), для всех $n \in \mathbb{Z}$. Обозначая через $q_{n}^{*}=q_{n}\left(t^{*}\right), v_{n}^{*}=\frac{d q_{n}}{d t}\left(t^{*}\right)$, получаем, что для начальных данных $\left\{\left(q_{n}^{*}\right),\left(v_{n}^{*}\right)\right\}$ аналитического решения уравнения (1.1) не существует. Теорема 1.1 доказана. 
Теорема 1.2. Предположим, что в уравнении (1.1) функиия $\varphi\left(q_{n}\right)$ имеет вид $\varphi\left(q_{n}\right)=-h \sin \left(\frac{2 \pi}{a} q_{n}\right)$, где константа $h$ удовлетворяет следующим неравенствам: $0<h<\frac{2 a}{\pi}, h \neq \frac{a}{\pi}\left(1-\cos \frac{2 \pi s}{l}\right)$ nрu $s=0, \pm 1, \ldots, \pm l ; l=1,2,3,4$. Тогда существуют такие константы $m_{n}^{0}>0$, что если при всех $n \in \mathbb{Z}$ массьи $m_{n}$ удовлетворяют неравенствам $m_{n} \leqslant m_{n}^{0}$, то существуют такие начальные данные $\left\{\left(q_{n}^{*}\right),\left(v_{n}^{*}\right)\right\} \in \Omega^{*}$, для которых решение уравнения (1.1) с граничными условиями (1.3) в классе бесконечно-дифферениируемых функций существует при всех $t \in \mathbb{R}$, но не единственно, а в классе аналитических функиий $q_{n}(t)$ для тех же начальных данных решение задачи (1.1), (1.3) не существует в сколь угодно малой окрестности значения $t^{*}$.

ДокАЗАТЕЛЬСтво. В основе доказательства лежит утверждение теоремы 1.10 и ее следствие 1.1 ( 55, гл. 1). В силу этого следствия и условий теоремы 1.2 для уравнения (1.1) справедливо утверждение теоремы 1.10, согласно которому если при некоторых $m_{n}^{0}>0$ выполнены неравенства $m_{n} \leqslant m_{n}^{0}$, то сушествует бесконечно много бесконечно-диффференщируемых решений $\left(q_{n}(t)\right)$ уравнения (1.1) таких, что при всех $n \in \mathbb{Z}, t \in \mathbb{R}$

$$
\left|q_{n}(t)-\left(n-\frac{1}{2}\right) a\right| \leqslant \varepsilon
$$

где $\varepsilon>0$ и не зависит от $n$ и $t$. При этом, так как каждое решение однозначно определяется парой функщий $\left(q_{0}(t), q_{1}(t)\right)$, удовлетворяюших $(1.4)$ и неравенствам $\left|\frac{d^{s} q_{\nu}}{d t^{s}}(t)\right|<\delta$, в которых $\nu=0,1 ; \delta>0 ; s=1, \ldots, k_{0}$, то, задавая две различные пары функций $\left(q_{0}(t), q_{1}(t)\right)$ и $\left(\widehat{q}_{0}(t), \widehat{q}_{1}(t)\right)$, удовлетворяюшие указанньм условиям, так, чтобы $q_{\nu}\left(t^{*}\right)=\widehat{q}_{\nu}\left(t^{*}\right), \frac{d^{k} q_{\nu}}{d t^{k}}\left(t^{*}\right)=\frac{d^{k} \widehat{q}_{\nu}}{d t^{k}}\left(t^{*}\right)(\nu=0,1 ; k=1,2, \ldots)$, получаем два различных решения уравнения (1.1) с одинаковьми начальньми данными из $\Omega^{*}$, удовлетворяюшие неравенству (1.4), которое обеспечивает выполнение граничных условий (1.3). Возможность несушествования аналитических решений в этой ситуации доказьвается так же, как и в теореме 1.1. Теорема 1.2 доказана.

ЗАмЕчАнИЕ 1.1. Из доказательства теоремы 1.2 и теоремы 1.10 следует, что утверждение теоремы 1.2 справедливо для функции $\varphi(t)$ общего вида.

\section{$\S$ 2. Введение фазовых пространств и однозначная разрешимость уравнения в классах бесконечно-дифференцируемых и аналитических функций}

ОПРЕДЕЛЕНИЕ 1.2. Введем пространство $\Omega$, состоящее из всевозможных пар $\left(q_{0}^{*}(t), q_{1}^{*}(t)\right)$ бесконечно-дифференцируемых функций $q_{0}^{*}(t), q_{1}^{*}(t)$ на прямой $t \in \mathbb{R}$.

ОПРЕДЕЛЕНИЕ 1.3. Пусть $r>0$-вешественное число. Введем пространство $\Omega_{r}$, состоящее из всевозможных пар $\left(q_{0}^{*}(t), q_{1}^{*}(t)\right)$ функций $q_{0}^{*}(t), q_{1}^{*}(t)$, аналитических в области $\left|t-t^{*}\right|<r$, и пространство $\Omega_{\infty}$, состояшее из пар $\left(q_{0}^{*}(t), q_{1}^{*}(t)\right)$ целых аналитических функций $q_{0}^{*}(t), q_{1}^{*}(t)$. 
ОПРЕДЕЛЕНИЕ 1.4. Определим подпространство $\Omega_{r}^{*} \subset \Omega^{*}$ пространства $\Omega^{*}$, введенного в определении $1.1(\S 1)$, точкой которого $\left\{\left(q_{n}^{*}\right),\left(v_{n}^{*}\right)\right\}$ являются такие начальные данные из пространства $\Omega^{*}$, для которых существует решение уравнения (1.1), аналитическое в области $\left|t-t^{*}\right|<r$, а также введем пространство $\Omega_{\infty}^{*}=\bigcap_{r>0} \Omega_{r}^{*}$.

Теорема 1.3 (существование и единственность решения в пространстве $\Omega$ ). Для любой пары функиий $\left(q_{0}^{*}(t), q_{1}^{*}(t)\right) \in \Omega$ для всех $n \in \mathbb{Z}$ существуют и однозначно определяются такие бесконечно-дифференцируемые на прямой $t \in \mathbb{R}$ функции $q_{n}(t)$, удовлетворяющие уравнению (1.1), что выполняются равенства

$$
q_{0}(t) \equiv q_{0}^{*}(t), \quad q_{1}(t) \equiv q_{1}^{*}(t) .
$$

Доказательство теоремы 1.3 непосредственно следует из вида уравнения (1.1).

ТЕорема 1.4 (усиленная теорема о единственности решений в пространстве $\Omega$ ). Пусть даны два бесконечно-дифференчируемых решения $\left(q_{n}^{\prime}(t)\right),\left(q_{n}^{\prime \prime}(t)\right)$ уравнения (1.1) такие, что парь функиий $\left(q_{0}^{\prime}(t), q_{1}^{\prime}(t)\right)$ и $\left(q_{0}^{\prime \prime}(t), q_{1}^{\prime \prime}(t)\right)$ не совпадают. Тогда существует такое челое число $n \neq 0,1$, что $q_{n}^{\prime}(t) \neq q_{n}^{\prime \prime}(t)$.

ДокАЗАТЕльство. Предположим противное, т.е. что при всех $n \neq 0,1$ и $t \in \mathbb{R}$ имеют место равенства

$$
q_{n}^{\prime}(t)=q_{n}^{\prime \prime}(t)
$$

Рассмотрим два случая:

1) $q_{1}^{\prime}(t) \equiv q_{1}^{\prime \prime}(t)$

2) $q_{1}^{\prime}(t) \not \equiv q_{1}^{\prime \prime}(t)$.

В случае 1$)$ имеем: $q_{0}^{\prime}(t) \not \equiv q_{0}^{\prime \prime}(t)$, и поэтому в силу $(1.1) q_{2}^{\prime}(t) \not \equiv q_{2}^{\prime \prime}(t)$, что противоречит (1.5).

В случае же 2) в силу (1.5) имеем: $q_{2}^{\prime}(t) \equiv q_{2}^{\prime \prime}(t)$, и поэтому в силу (1.1) получаем неравенство $q_{3}^{\prime}(t) \not \equiv q_{3}^{\prime \prime}(t)$, что противоречит (1.5). Теорема 1.4 доказана.

Tеорема 1.5. Для любого конечного значения $r>0$ и для $r=\infty$ между пространствами $\Omega_{r}^{*}$ и $\Omega_{r}$ существует взаимно-однозначное соответствие $S: \Omega_{r}^{*} \rightarrow \Omega_{r}$, и в них уравнение (1.1) однозначно разрешимо в классе аналитических функиий, т.е. для любьх начальных данных в пространствах $\Omega_{r}$ и $\Omega_{r}^{*}$ существует единственное решение уравнения (1.1) в области $\left|t-t^{*}\right|<r$, которое в этой области аналитично.

ДоКАЗАТЕЛЬСТВо. Построим взаимно-однозначное отображение $S$ и обратное к нему отображение $S^{-1}: \Omega_{r} \rightarrow \Omega_{r}^{*}$, при которых начальные данные для уравнения (1.1) соответствуют друг другу. Пусть $\left\{\left(q_{n}^{*}\right),\left(v_{n}^{*}\right)\right\} \in \Omega_{r}^{*}$. Согласно определениям $1.4,1.1$ и теореме $1.1(\S 1)$, в области $\left|t-t^{*}\right|<r$ существует единственное аналитическое решение $\left(q_{n}(t)\right)$ уравнения (1.1) такое, что при всех $n \in \mathbb{Z} q_{n}\left(t^{*}\right)=q_{n}^{*}, \frac{d q_{n}}{d t}\left(t^{*}\right)=v_{n}^{*}$. Поэтому полагаем

$$
S\left\{\left(q_{n}^{*}\right),\left(v_{n}^{*}\right)\right\}=\left(q_{0}(t), q_{1}(t)\right) \in \Omega_{r} .
$$

Обратно, пусть $\left(q_{0}^{*}(t), q_{1}^{*}(t)\right) \in \Omega_{r}$. В силу определения 1.3 функции $q_{0}^{*}(t)$ и $q_{0}^{*}(t)-$ аналитические в области $\left|t-t^{*}\right|<r$, и поэтому из вида уравнения (1.1) следует, что 
если положить $q_{0}(t) \equiv q_{0}^{*}(t), q_{1}(t) \equiv q_{1}^{*}(t)$, то при всех $n \in \mathbb{Z}$ однозначно восстанавливаются аналитические в области $\left|t-t^{*}\right|<r$ функции $q_{n}(t)$, удовлетворяющие уравнению (1.1). Полагая теперь

$$
S^{-1}\left(q_{0}^{*}(t), q_{1}^{*}(t)\right)=\left\{\left(q_{n}^{*}\right),\left(v_{n}^{*}\right)\right\} \in \Omega_{r}^{*},
$$

где при $n \in \mathbb{Z} q_{n}^{*}=q_{n}\left(t^{*}\right), v_{n}^{*}=\frac{d q_{n}}{d t}\left(t^{*}\right)$, получаем утверждение теоремы 1.5 . Tеорема 1.5 доказана.

Так как нас интересуют решения уравнения (1.1) при всех $t$ на прямой $t \in \mathbb{R}$, то в дальнейшем мы будем изучать поведение решений $\left(q_{n}(t)\right)$ только в двух случаях:

а) в классе бесконечно-дифференцируемых функций $\left(q_{n}(t)\right)$ с начальными данными из фазового пространства $\Omega$;

б) в классе целых аналитических функций на прямой $t \in \mathbb{R}$ c начальньми данными из пространства $\Omega_{\infty}$ или из пространства $\Omega_{\infty}^{*}$.

Пространства $\Omega$ и $\Omega_{\infty}$ являются линейными в естественном смысле: если $\left(q_{0}^{\prime}(t), q_{1}^{\prime}(t)\right) \in \Omega, \quad\left(q_{0}^{\prime \prime}(t), q_{1}^{\prime \prime}(t)\right) \in \Omega$, то $\left(q_{0}^{\prime}(t)+q_{0}^{\prime \prime}(t), q_{1}^{\prime}(t)+q_{1}^{\prime \prime}(t)\right) \in \Omega$, а для любого вешественного числа $\lambda$ точка $\left(\lambda q_{0}^{\prime}(t), \lambda q_{1}^{\prime}(t)\right) \in \Omega$, и те же утверждения справедливы, если заменить пространство $\Omega$ на $\Omega_{\infty}$. Для исследования устойчивости стационарных решений в $\S \S 5,6$ нам потребуются следуюшие понятия, которые вводятся в определениях $1.5,1.6$ и 1.7 .

ОпРЕДЕлЕниЕ 1.5. Для любого целого $k \geqslant 0$ и любого элемента $\widetilde{q}=\left(q_{0}(t), q_{1}(t)\right)$, принадлежащего пространству $\Omega$ или его подпространству $\Omega_{\infty}$, введем норму

$$
\|\widetilde{q}\|^{(k)}=\left\|\left(q_{0}(t), q_{1}(t)\right)\right\|^{(k)}=\sum_{\nu=0}^{1} \sum_{s=0}^{k} \sup _{t \in \mathbb{R}}\left|\frac{d^{s} q_{\nu}}{d t^{s}}(t)\right|,
$$

где по определению $\frac{d^{0} q_{\nu}}{d t^{0}}(t)=q_{\nu}(t)$.

ОПРЕДЕлЕНИЕ 1.6. Для любого целого числа $k \geqslant 0$ введем расстояние $\rho^{(k)}(\cdot, \cdot)$ в пространстве $\Omega_{\infty}^{*}$ следуюшим образом. Если $q^{\prime}=\left\{\left(q_{n}^{\prime}\right),\left(v_{n}^{\prime}\right)\right\} \in \Omega_{\infty}^{*}, q^{\prime \prime}=$ $\left\{\left(q_{n}^{\prime \prime}\right),\left(v_{n}^{\prime \prime}\right)\right\} \in \Omega_{\infty}^{*}$, то

$$
\rho^{(k)}\left(q^{\prime}, q^{\prime \prime}\right)=\left\|S q^{\prime}-S q^{\prime \prime}\right\|^{(k)},
$$

где $S$ - отображение, введенное в теореме 1.5 .

ОПРЕДЕЛЕНИЕ 1.7. Пусть $\kappa=\left(\kappa_{0}, \kappa_{1}, \ldots\right)$ - односторонняя последовательность, состояшая из положительных чисел $\kappa_{s}(s=0,1 \ldots), q^{0}=\left(q_{n}^{0}(t)\right)$ - аналитическое решение уравнения (1.1), $\widetilde{q}^{0}=\left(q_{0}^{0}(t), q_{1}^{0}(t)\right) \in \Omega_{\infty}, \widehat{q}^{0}=\left\{\left(\widehat{q}_{0}^{0}\right),\left(\widehat{v}_{n}^{0}\right)\right\} \in \Omega_{\infty}^{*}-$ такой элемент пространства $\Omega_{\infty}^{*}$, что при всех $n \in \mathbb{Z} \widehat{q}_{n}^{0}=q_{n}^{0}\left(t^{*}\right), \widehat{v}_{n}^{0}=\frac{d q_{n}^{0}}{d t}\left(t^{*}\right)$. В пространствах $\Omega, \Omega_{\infty}$ и $\Omega_{\infty}^{*}$ введем множества $\Omega^{(\kappa)}\left(q^{0}\right) \subset \Omega, \Omega_{\infty}^{(\kappa)}\left(q^{0}\right) \subset \Omega_{\infty}, \Omega_{\infty}^{*(\kappa)}\left(q^{0}\right) \subset \Omega_{\infty}^{*}$ следуюшим образом:

$$
\begin{aligned}
\Omega^{(\kappa)}\left(q^{0}\right) & =\left\{q^{*}=\left(q_{0}^{*}(t), q_{1}^{*}(t)\right) \in \Omega:\left\|q^{*}-\widetilde{q}^{0}\right\|^{(k)} \leqslant \kappa_{k} ; k=0,1,2, \ldots\right\}, \\
\Omega_{\infty}^{(\kappa)}\left(q^{0}\right) & =\left\{q^{*}=\left(q_{0}^{*}(t), q_{1}^{*}(t)\right) \in \Omega_{\infty}:\left\|q^{*}-\widetilde{q}^{0}\right\|^{(k)} \leqslant \kappa_{k} ; k=0,1,2, \ldots\right\}, \\
\Omega_{\infty}^{*(\kappa)}\left(q^{0}\right) & =\left\{\widehat{q}=\left\{\left(\widehat{q}_{n}\right),\left(\widehat{v}_{n}\right)\right\} \in \Omega_{\infty}^{*}: \rho^{(k)}\left(\widehat{q}, \widehat{q}^{0}\right) \leqslant \kappa_{k} ; k=0,1,2, \ldots\right\} .
\end{aligned}
$$


§3. Построение семейств периодических решений, решений, имеющих тип бегущей волны, и задача Френкеля-Конторовой

Дадим четыре основные для этого параграфа определения решений уравнения (1.1).

ОПРеДЕЛЕнИЕ 1.8. Решение $\left(q_{n}(t)\right)$ назьвается стационарным, если оно не зависит от времени, т.е. при всех $n \in \mathbb{Z} q_{n}(t) \equiv q_{n}^{0}$ - константа, не зависящая от $t$.

ПримеР 1.1. Пусть $\eta$ - такое значение, что $\varphi(\eta)=0$, а $k$ - целое число. Тогда решение $\left(q_{n}(t)\right)$, в котором $q_{n}(t) \equiv \eta+n k a$, будет стационарным.

ОПредЕЛЕниЕ 1.9. Решение $\left(q_{n}(t)\right)$ назьвается пространственно однородным, если существует такое число $\eta$, не зависяшее от $n$ и $t$, что движение $q_{n}(t)$ частицы $P_{n}$ (см. введение), определяемое этим решением, относительно положения $q_{n}^{0}=n a+\eta$, одинаково для всех $n \in \mathbb{Z}$.

ПримеР 1.2. Решение примера 1.1 при $k=1$ является пространственно однородным.

ОПРЕДЕЛЕНИЕ 1.10. Решение $\left(q_{n}(t)\right)$ называется периодическим, если существует такая константа $T>0$, не зависящая от $t$ и $n$ и называемая периодом, что при всех $n \in \mathbb{Z}$ и $t \in \mathbb{R}$ выполнено равенство $q_{n}(t+T)=q_{n}(t)$. Если при этом величина $2 b=$ $\max _{0 \leqslant t \leqslant T} q_{n}(t)-\min _{0 \leqslant t \leqslant T} q_{n}(t)$ не зависит от $n$, то число $b$ назьвается амплитудой колебаний.

ОПРЕДЕЛЕНИЕ 1.11. Будем говорить, что решение $\left(q_{n}(t)\right)$ имеет тип бегущей волны, если существует такая константа $\tau$, не зависящая от $t$ и $n$, что при всех $n \in \mathbb{Z}$, $t \in \mathbb{R}$ выполнено равенство

$$
q_{n}(t)=q_{n+1}(t+\tau)
$$

ПримеР 1.3. Решение примера 1.1 при $k=0$ является решением, имеюшим тип бегушей волны с любым $\tau$.

Примеры 1.1-1.3 доставляют тривиальные примеры периодических, пространственно однородных решений и решений, имеющих тип бегушей волны. Главная цель этого параграффа состоит в нахождении нестационарных решений указанных видов. Далее будут построены три принципиально различных семейства таких решений и сформулированы нерешенные проблемы о сушествовании других решений, отличных от найденных.

При построении этих семейств мы будем считать, что все константы $m_{n}$ в уравнении (1.1) одинаковые и равны $m>0$, и первоначально предположим, что функция $\varphi\left(q_{n}\right)$ в уравнении $(1.1)$ имеет вид $\varphi\left(q_{n}\right)=-h \sin \frac{2 \pi q_{n}}{a}($ теоремы $1.6,1.7)$, так как для этого случая можно получить более конкретные решения и именно он рассматривался Френкелем и Конторовой в работе [1] при постановке проблемы. Далее, в теоремах 1.8 и 1.9 мы укажем обшие условия на функцию $\varphi(q)$, при которых основные из описанных в теоремах 1.6 и 1.7 результатов, оказываются справедливыми. 
Подставляя в уравнение (1.1) вместо значений $m_{n}$ величину $m$, а вместо функции $\varphi\left(q_{n}\right)$ функцию $\varphi\left(q_{n}\right)=-h \sin \frac{2 \pi q_{n}}{a}$, получим следующее уравнение:

$$
m_{n} \frac{d^{2} q_{n}}{d t^{2}}=-h \sin \frac{2 \pi q_{n}}{a}+q_{n+1}-2 q_{n}+q_{n-1}, \quad n \in \mathbb{Z}
$$

ТеОРема 1.6. Уравнение (1.7) обладает двумя семействами решений $Q_{1}$ и $Q_{2}$ такими, что:

1) любое решение $\left(q_{n}(t)\right) \in Q_{1}$ является периодическим, имеет тип бегущей волны и удовлетворяет равенству (1.6), в котором мнохсество значений $\tau$ содержит бесконечный интервал

$$
\sqrt{\frac{2 \pi m a}{h}}<\tau<\infty
$$

2) если $q_{n}(t) \in Q_{1}$, то при любом $n \in \mathbb{Z} q_{n}(t) \not \equiv$ const, $a\left(n a+q_{n}(t)\right)$ - периодическое, пространственно однородное решение уравнения (1.7), причем его амплитуда колебаний не превосходит а/2 (рис. 1.1);

3) если $\left(q_{n}(t)\right) \in Q_{2}$, то $\left(\right.$ па $\left.+q_{n}(t)\right)$ - пространственно однородное решение уравнения (1.7), которое периодическим не является.

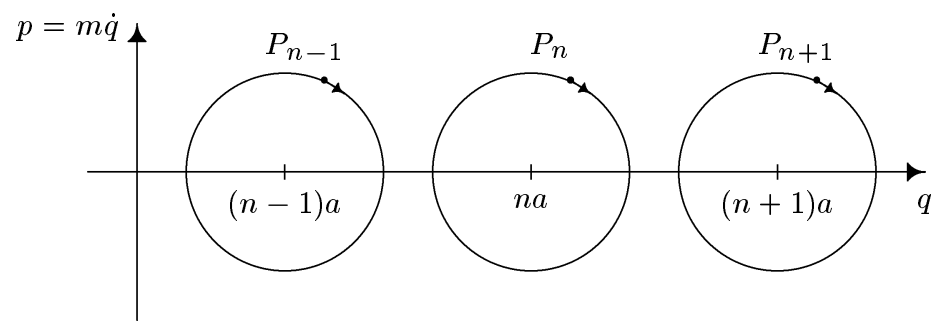

Рис. 1.1. Пространственно однородное движение частиц на фазовой плоскости $q, p$

ДокАЗАТЕЛЬСТво. Будем искать такое решение $\left(q_{n}(t)\right)$ уравнения $(1.7)$, для которого при любых $n \in \mathbb{Z}, t \in \mathbb{R}$ выполнено условие

$$
q_{n}(t)=q_{n+1}(t)=q(t)
$$

Тогда в силу (1.7) функция $q(t)$ удовлетворяет уравнению

$$
m \frac{d^{2} q}{d t^{2}}=-h \sin \frac{2 \pi q}{a}
$$

Уравнение (1.9) есть уравнение плоского нелинейного маятника [12], и утверждения теоремы 1.6 следуют из свойств уравнения (1.9). При этом решения уравнения (1.7), 
принадлежащие семейству $Q_{2}$, удовлетворяют равенству $(1.8)$, в котором $q(t)$ - неограниченное решение уравнения (1.9), и для этого случая выполняется утверждение 3 ) теоремы 1.6; решения уравнения (1.7), принадлежашие семейству $Q_{1}$, удовлетворяют равенству (1.8), в котором $q(t)$ - периодическое решение уравнения (1.9), а значение $\tau$ равно периоду этого периодического решения. Из теории уравнения нелинейного маятника известно, что при стремлении к положению равновесия $q=0$ период периодических решений стремится к величине $\tau_{0}=\sqrt{\frac{2 \pi m a}{h}}$, которая является периодом колебаний плоского линейного маятника, описываемого уравнением $m \frac{d^{2} q}{d t^{2}}=-h \frac{2 \pi q}{a}$, а при стремлении к сепаратрисе период колебаний нелинейного маятника стремится к бесконечности. Так как при этом периодическое решение $q_{n}(t)$ уравнения (1.7) с периодом $\tau$, удовлетворяюшее равенству (1.8), является решением, имеющим тип бегущей волны, для которого выполнено (1.6), то из неравенства $\tau_{0}<\tau<\infty$ следует утверждение 1$)$ теоремы 1.6. Из свойств решений уравнения (1.9) также следует, что амплитуда колебаний периодических решений $q(t) \not \equiv 0$ уравнения (1.9) не превосходит $a / 2$, а так как в силу (1.8) и определения 1.9 для решения $q(t)$ уравнения (1.9) решение $(n a+q(t))$ есть пространственно однородное решение уравнения (1.7), то вьполняются утверждения 2) и 3) теоремы 1.6. Теорема 1.6 доказана.

Теорема 1.7. Уравнение (1.7) обладает семейством решений $Q$ таким, что

1) любое решение $\left(q_{n}(t)\right) \in Q$ является периодическим, имеет тип бегущей волны и условие (1.6) выполнено с некоторым $\tau>0$;

2) если при некотором $\xi$ параметр $h$ в уравнении (1.7) удовлетворяет соотношениям

$$
\sin \frac{2 \pi \xi}{a}=-\frac{4 \xi}{h}, \quad \cos \frac{2 \pi \xi}{a}<-\frac{2 a}{h \pi}
$$

то множество значений $\tau$ для решений из $Q$ содержит бесконечный интервал $\widetilde{k} \leqslant \tau<\infty$, где $\widetilde{k}-$ некоторая константа;

3) если $q_{n}(t) \in Q$, то при любом $n \in \mathbb{Z} q_{n}(t) \not \equiv$ const, $q_{n}(t) \equiv-q_{n+1}(t)$, $a\left(\right.$ па $\left.+q_{n}(t)\right)$ - периодическое не являющееся пространственно однородным решение уравнения (1.7), амплитуда колебаний которого может быть сколь угодно большой (рис. 1.2).

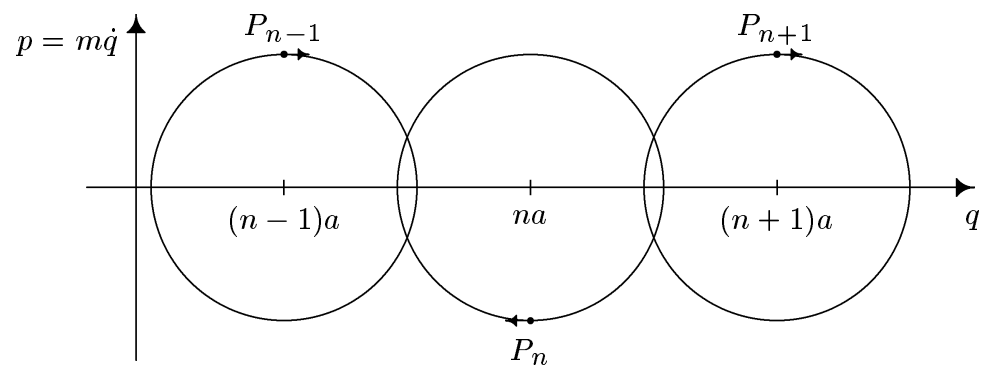

Рис. 1.2. Обменная диффузия на фазовой плоскости $q, p$ 
ЗАмЕчАниЕ 1.2. Если величина $h$ - достаточно большая, то в области $\frac{1}{2} a<\xi<\frac{3}{4} a$ всегда найдется такое $\xi$, для которого вьполнены соотношения (1.10).

ДокАЗАТЕльство. Будем искать такое решение $\left(q_{n}(t)\right)$ уравнения $(1.7)$, для которого при любых $n \in \mathbb{Z}, t \in \mathbb{R}$ выполнено равенство

$$
q_{n}(t)=-q_{n+1}(t) .
$$

Тогда в силу (1.7) и нечетности функщии $\sin \frac{2 \pi q_{n}}{a}$ при любом $n$ функция $q_{n}(t)$ удовлетворяет уравнению

$$
m \frac{d^{2} q_{n}}{d t^{2}}=-h \sin \frac{2 \pi q_{n}}{a}-4 q_{n}
$$

и любое решение $q_{n}(t)$ уравнения (1.12) порождает решение $\left(q_{n}(t)\right)$ уравнения $(1.7)$, удовлетворяющее условию (1.11). Уравнение (1.12) имеет потенщиал

$$
U\left(q_{n}\right)=-\frac{h a}{2 \pi} \cos \frac{2 \pi q_{n}}{a}+2 q_{n}^{2}
$$

такой, что

$$
m \frac{d^{2} q_{n}}{d t^{2}}=-\frac{\partial U}{\partial q_{n}}\left(q_{n}\right)
$$

В силу (1.13) $U\left(q_{n}\right)$ - четная функция, $\lim _{q_{n} \rightarrow \infty} U\left(q_{n}\right)=\infty, \frac{d U}{d q_{n}}>0$ при $q_{n}>h / 4$, в точке $q_{n}=0$ функция $U\left(q_{n}\right)$ достигает минимума, а, если выполнены соотношения (1.10), то в точке $q_{n}=\xi$ функция $U\left(q_{n}\right)$ имеет локальньй максимум. Поэтому из теории потенциальных систем с одной степенью свободы [12] следует сушествование такого числа $q^{*}$, что любому значению $\sigma>q^{*}$ соответствует периодическое решение $q_{n}(t)$ уравнения (1.14), имеюшую амплитуду колебаний, равную $\sigma$, некоторый период колебаний $T>0$ и полупериод $\tau=T / 2$ такой, что

$$
q_{n}(t+\tau) \equiv-q_{n}(t)
$$

Сравнивая теперь равенства (1.11) и (1.15), получаем, что это периодическое решение уравнения (1.14), являюшееся периодическим решением эквивалентного ему уравнения (1.12) и удовлетворяющее условию (1.15), удовлетворяет равенству (1.6) с $\tau=T / 2$, и, таким образом, решение $\left(q_{n}(t)\right)$ уравнения (1.7) имеет тип бегущей волны и при этом амплитуда колебаний $\sigma$ может быть сколь угодно большой. Из теории потенциальных систем с одной степенью свободы также следует, что такие же периодические решения, удовлетворяюшие равенству (1.15) и имеюшие амплитуду, равную $\sigma$, сушествуют для любого $\sigma$, принадлежащего интервалу $0 \leqslant \sigma<\xi$, где $\xi-$ наименьшее положительное значение, являюшее точкой локального максимума функции $U\left(q_{n}\right)$, и при этом период колебаний $T$ этих периодических решений стремится к бесконечности, если $\sigma \rightarrow \xi$. Так как соотношения (1.10) и замечание 1.2 гарантируют существование такого значения $\xi$, то, полагая в качестве $Q$ семейство решений $\left(q_{n}(t)\right)$ уравнения $(1.7)$, у которых $q_{n}(t)$ - указанные периодические решения уравнения (1.12), удовлетворяюшие равенствам (1.11) и (1.15), мы получаем все утверждения теоремы 1.7 , в которых $\tau=T / 2$. Теорема 1.7 доказана. 
ТЕОРема 1.8. Предположим, что в уравнении (1.1) при всех $n \in \mathbb{Z}$ параметры $m_{n}=m>0$, функиия $\varphi(q)$ удовлетворяет равенству $\int_{0}^{a} \varphi(q) d q=0$, а $\eta$ - такое значение, что $\varphi(\eta)=0$. Тогда уравнение (1.1) имеет два семейства решений $Q_{1}$ и $Q_{2}$ такие, что

1) любое решение $\left(q_{n}(t)\right) \in Q_{1}$ является периодическим, имеет тип бегущей волны и удовлетворяет равенству (1.6), в котором множсество значений $\tau$ содержит бесконечный интервал $\widetilde{k} \leqslant \tau<\infty ;$

2) если $\left(q_{n}(t)\right) \in Q_{1}$, то при любом $n \in \mathbb{Z} q_{n}(t) \not \equiv 0, a\left(\eta+n a+q_{n}(t)\right)-$ периодическое, пространственно однородное решение уравнения (1.1), амплитуда колебаний которого не превосходит константы, не зависящей om $n$;

3) если $\left(q_{n}(t)\right) \in Q_{2}$, то $\left(\eta+n a+q_{n}(t)\right)$ - пространственно однородное решение уравнения (1.1), которое периодическим не является.

ДокАЗАТЕльство. Так же, как и при доказательстве теоремы 1.6, будем искать такое решение $\left(q_{n}(t)\right)$ уравнения $(1.1)$, для которого при любом $n \in \mathbb{Z}$ вьполнено равенство (1.8). Тогда функция $q(t)$ удовлетворяет уравнению

$$
m \frac{d^{2} q}{d t^{2}}=\varphi(q)=-\frac{\partial f}{\partial q}(q)
$$

где $f(q)=-\int_{\eta}^{q} \varphi(y) d y$. Уравнение (1.16) с гладкой периодической функцией $f(q)$ есть уравнение маятникового типа, которое имеет стационарные решения (положения равновесия) в точках $\widetilde{q}$, где $\varphi(\widetilde{q})=0$.

Рассмотрим значение $q=q^{\prime}$, где функция $f(q)$ достигает минимума, и ближайшее к $q^{\prime}$ значение $q^{\prime \prime}$, где функция $f(q)$ имеет локальньй максимум. В достаточно малой окрестности $q^{\prime}$ решения уравнения (1.16) являются периодическими, и для них соответствуюшее решение $\left(q_{n}(t)\right)$ уравнения (1.1) имеет тип бегушей волны, а число $\tau$ в равенстве (1.6) есть период периодического решения $q_{n}(t)$. При приближении к точке $q=q^{\prime \prime}$ период периодического решения $q(t)$ уравнения (1.16) стремится к бесконечности, и поэтому множество значений $\tau$, для которых соответствуюшие решения $\left(q_{n}(t)\right)$ уравнения (1.1) удовлетворяют равенству (1.6), содержит бесконечный интервал $\widetilde{k} \leqslant \tau<\infty$. Обозначая через $Q_{1}$ множество этих решений $\left(q_{n}(t)\right)$, мы получаем утверждения 1) и 2) теоремы 1.8. Уравнение (1.16) имеет также неограниченные решения, которые периодическими не являются, и, обозначая через $Q_{2}$ множество соответствуюших решений уравнения (1.1), мы в силу (1.8) получаем утверждение 3 ) теоремы 1.8. Теорема 1.8 доказана.

ТЕОРема 1.9. Предположим, что в уравнении (1.1) при всех $n \in \mathbb{Z}$ параметры $m_{n}=m>0$ и справедливо равенство

$$
\varphi(q) \equiv-\varphi(-q)
$$

Тогда уравнение (1.1) обладает семейством решений $Q$ таким, что

1) любое решение $\left(q_{n}(t)\right) \in Q$ является периодическим и имеет тип бегущей волнын; 
2) если $\left(q_{n}(t)\right) \in Q$, то при любом $n \in \mathbb{Z} \quad q_{n}(t) \not \equiv 0, q_{n}(t) \equiv-q_{n+1}(t)$, $a\left(\right.$ па $\left.+q_{n}(t)\right)$ - периодическое решение, не являющееся пространственно однородным, амплитуда колебаний которого может быть сколь угодно больиой.

ДокАЗАТЕльство. Повторяя доказательство теоремы 1.7, будем искать такое решение $\left(q_{n}(t)\right)$ уравнения (1.1), что выполнено равенство (1.11). В этом случае в силу $(1.1)$ и (1.17) при любом $n \in \mathbb{Z}$ функция $q_{n}(t)$ удовлетворяет уравнению

$$
m \frac{d^{2} q_{n}}{d t^{2}}=\varphi\left(q_{n}\right)-4 q_{n}
$$

и любое решение уравнения (1.18) порождает решение уравнения (1.1), удовлетворяюшее (1.11). Уравнение (1.18) можно представить в потенциальной форме (1.14), где потенциал $U\left(q_{n}\right)$ имеет вид $U\left(q_{n}\right)=-\int_{0}^{q_{n}} \varphi(q) d q+2 q_{n}^{2}$. В силу $(1.17) U\left(q_{n}\right)-$ четная функция и существует такое значение $q^{*}$, что для любого $\sigma>q^{*}$ сушествует периодическое решение $q_{n}(t) \not \equiv 0$ уравнения (1.18), имеющее амплитуду колебаний, равную $\sigma$, период колебаний $T>0$ и полупериод $\tau=T / 2$ такой, что справедливо равенство (1.15).

Так как в силу (1.17) $\varphi(0)=0$, то утверждения 1) и 2) теоремы 1.9 очевидно следуют из равенств (1.11) и (1.15). Теорема 1.9 доказана.

В основополагающей работе [1] Я. И. Френкель и Т. А. Конторова поставили задачу изучения движения счетного числа точек $P_{n}$ на прямой, взаимодействуюших между собой с помошњю потенциала

$$
U=\gamma \sum_{n \in \mathbb{Z}} f\left(q_{n}\right)+\chi \sum_{n \in \mathbb{Z}}\left(q_{n+1}-q_{n}-a\right)^{2},
$$

где $n$ - целое число, $q_{n}$ - координата точки $P_{n}$ на прямой, $f(q)$ - бесконечно-дифференщируемая функция, имеюшая по $q$ период $a>0$, которая в точках $q=n a$ имеет минимум, а в точках $q=\left(n+\frac{1}{2}\right) a$ - максимум, $\gamma>0, \chi>0$-параметры. Физически это означает, что система представляет собой цепочку пружинок, находяшихся во внешнем поле с потенциалом $\gamma f(q)$ (рис. 1.3).

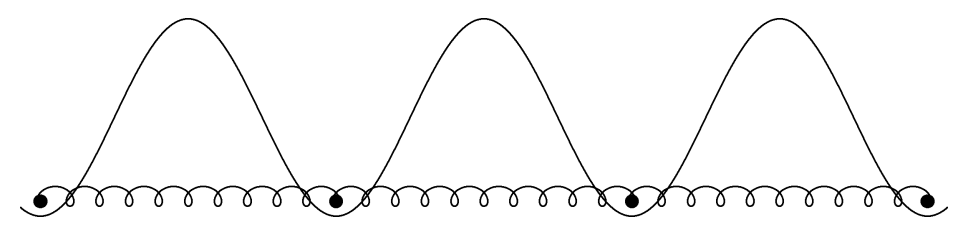

Рис. 1.3. Цепочка пружинок во внешнем периодическом поле

Если обозначить массу частищы $P_{n}$ через $M_{n}$, ее скорость - через $v_{n}$, а импульсчерез $p_{n}=M_{n} v_{n}$, то гамильтониан $H$ (см. $\S 7$, гл. 1$)$ примет вид

$$
H=\sum_{n \in \mathbb{Z}} \frac{p_{n}^{2}}{2 M_{n}}+U,
$$


и поэтому, полагая $\psi(q)=\frac{d f}{d q}(q)$, получим, что движение частиц во времени $t$ описывается с помошью следуюшей бесконечной системы уравнений

$$
M_{n} \frac{d^{2} q_{n}}{d t^{2}}=-\gamma \psi\left(q_{n}\right)+2 \chi\left(q_{n+1}-2 q_{n}+q_{n-1}\right), \quad n \in \mathbb{Z},
$$

которые совпадают с уравнением (1.1), если положить

$$
m_{n}=\frac{M_{n}}{2 \chi}, \quad \varphi(q)=-\frac{\gamma}{2 \chi} \psi(q) .
$$

В случае, когда массы всех частиц $P_{n}$ одинаковые и равны $M>0$, a $f(q)=1-\cos \frac{2 \pi q}{a}$, эта система уравнений впервые была выписана в работе [1], в которой изучалась следуюшая задача. Рассмотрим движение частиц в окрестности стационарного решения $q_{n}(t) \equiv n a, n \in \mathbb{Z}$. С этой целью введем неизвестные величины $\xi_{n}$ с помощю равенств $q_{n}=n a+\xi_{n}$ и перепишем для указанного частного случая систему уравнений (1.19) через переменные $\xi_{n}$ в виде системы уравнений

$$
M \frac{d^{2} \xi_{n}}{d t^{2}}=-\frac{2 \pi \gamma}{a} \sin \frac{2 \pi \xi_{n}}{a}+2 \chi\left(\xi_{n+1}-2 \xi_{n}+\xi_{n-1}\right), \quad n \in \mathbb{Z},
$$

которые совпадают с уравнениями (1.7), если в них положить $q_{n}=\xi_{n}, m=\frac{M}{2 \chi}$, $h=\frac{\pi \gamma}{a \chi}$.

Задача Френкеля-Конторовой состоит в следуюшем: требуется найти решение $\xi_{n}(t) \not \equiv 0(n \in \mathbb{Z})$ системы $(1.20)$, удовлетворяюшее при всех $n \in \mathbb{Z}, t \in \mathbb{R}$ равенствам

$$
\xi_{n}(t)=\xi_{n+1}(t+\tau),
$$

где $\tau$ - константа, не зависящая от $t$ и $n$, удовлетворяюшая условию

$$
M<2 \chi \tau^{2} .
$$

Впервые строгое решение этой задачи получено в работе [41] и отражено в теоремax 1.6 и 1.7 этого параграффа.

Действительно, согласно определению 1.11 и условиям (1.20) и $(1.21), \xi_{n}(t)$ является решением, имеюшим тип бегушей волны, а в теоремах 1.6 и 1.7 были найдены два различных семейства $Q_{1}$ и $Q$ таких решений. При этом, согласно утверждению 1) теоремы 1.6 и утверждению 2) теоремы 1.7, множество значений $\tau$ в равенстве (1.21) содержит бесконечный интервал $\widetilde{k} \leqslant \tau<\infty$, и, следовательно, существует такое число $k^{*}>\widetilde{k}$, что значения $\tau$, расположенные в интервале $k^{*} \leqslant \tau<\infty$, удовлетворяют как равенству (1.21), так и неравенству (1.22). Таким образом, задача Френкеля-Конторовой полностью решена. Отметим, что в случае семейства $Q_{1}$, согласно утверждению 2) теоремы 1.6, амплитуда колебаний решения $q_{n}(t)=n a+\xi_{n}(t)(n \in \mathbb{Z})$ системы уравнений (1.19) не превосходит $a / 2$ (рис. 1.1), а в случае семейства $Q$, согласно утверждению 3$)$ теоремы 1.7 , амплитуда колебаний решения $q_{n}(t)$ может быть сколь угодно большой (рис. 1.2). В результате возникает явление обменной диффузии, при котором порядок расположения частиц на прямой периодически меняется (рис. 1.2).

В связи с задачей Френкеля-Конторовой и результатами этого параграф̆а (теоремы 1.6-1.9) представляется естественным сформулировать некоторые нерешенные в настоящее время проблемы, касающиеся решений, имеющих тип бегущей волны. 
ПроБЛемА 1.1. Существует ли хотя бы одно решение уравнений (1.1) и (1.7), имеющее тип бегущей волнь, отличное от следующих:

а) $q_{n}(t) \equiv \eta$, где $n \in \mathbb{Z}, \varphi(\eta)=0$ (пример 1.3$)$;

б) $\left(q_{n}(t)\right) \in Q_{1},\left(q_{n}(t)\right) \in Q$, где $Q_{1}$ - семейство решений, построенное в теоремах 1.6 и 1.8, а $Q$ - семейство, построенное в теоремах 1.7 и 1.9;

в) $\left(\eta+q_{n}(t)\right)$, где $\varphi(\eta)=0$, а решение $\left(q_{n}(t)\right) \in Q_{1}$ или $\left(q_{n}(t)\right) \in Q$.

ПроБлемА 1.2. Найти все решения уравнений (1.1) и (1.7), имеющих тип бегущей волны.

\section{$\S$ 4. Неустойчивость стационарных и периодических решений в случае равных масс}

Цель этого параграфа состоит в том, чтобы показать, что в случае, когда все массы $m_{n}=m>0$, стационарные решения из примера $1.1 \S 3$ и периодические решения, построенные в теоремах 1.6 и $1.8 \S 3$ и принадлежашие семейству $Q_{1},-$ неустойчивые относительно изменения начальных данных в пространствах $\Omega, \Omega_{\infty}$ и $\Omega_{\infty}^{*}$, определенных в $\oint 2$. Применительно к пространству $\Omega_{\infty}^{*}$ это означает, что малое изменение начальных данных $\left\{\left(q_{n}^{*}\right),\left(v_{n}^{*}\right)\right\} \in \Omega_{\infty}^{*}$ в смысле расстояния $\rho^{(k)}(\cdot, \cdot)$ (определение $\left.1.6 \S 2\right)$ при любом целом $k \geqslant 0$ может приводить к большому изменению функций $q_{n}(t)$, составляюших решение $\left(q_{n}(t)\right)$ уравнения

$$
m \frac{d^{2} q_{n}}{d t^{2}}=\varphi\left(q_{n}\right)+q_{n+1}-2 q_{n}+q_{n-1}, \quad n \in \mathbb{Z},
$$

а применительно к пространствам $\Omega$ и $\Omega_{\infty}$ это означает, что малое изменение соответствующих начальных данных $\left(q_{0}^{*}(t), q_{1}^{*}(t)\right)$ в смысле нормы $\|\cdot\|^{(k)}$ (определение $\left.1.5 \S 2\right)$ при любом целом $k \geqslant 0$ также может приводить к большому изменению функщий $q_{n}(t)$ для достаточно больших $|n|$.

Для доказательства этих утверждений обозначим через $\left(\widetilde{q}_{n}(t)\right)$ указанное вьше стационарное или периодическое решение уравнения (1.23), неустойчивость которого мы хотим установить, и введем преобразование

$$
I:\left(q_{n-1}(t), q_{n}(t)\right) \rightarrow\left(q_{n}(t), q_{n+1}(t)\right)
$$

которое в силу (1.23) имеет следуюший вид:

$$
I(x(t), y(t))=\left(x^{\prime}(t), y^{\prime}(t)\right),
$$

где

$$
x^{\prime}(t)=y(t), \quad y^{\prime}(t)=-x(t)+2 y(t)-\varphi(y(t))+m \frac{d^{2} y}{d t^{2}}(t) .
$$

Если теперь $I$ линеаризовать в точке, соответствуюшей решению $\left(\widetilde{q}_{n}(t)\right)$, то в силу (1.24) получим, что линейная часть $d I$ в этой точке имеет вид $d I(d x, d y)=\left(d x^{\prime}, d y^{\prime}\right)$, где

$$
d I=\left(\begin{array}{cc}
0 & 1 \\
-1 & 2-\Delta^{2}
\end{array}\right)-\text { операторная матрица, }
$$


в которой $\Delta^{2}=-m \frac{d^{2}}{d t^{2}}+\frac{d \varphi}{d q}(\widetilde{q}(t))$ - оператор Шрёдингера с периодическим потенциалом, а $\widetilde{q}(t)$ периодическая функция, которая в силу определения решения $\left(\widetilde{q}_{n}(t)\right)$ не зависит от $n$. Так как спектр оператора $\Delta^{2}$ у ходит на бесконечность, то неподвижная точка $(0,0)$ будет гиперболической относительно действия матрицы $d I(t)$, которая получается из оператора $d I$, если его рассматривать как матрищу, зависящую от $t$ в плоскости, натянутой на $d x(t)$ и $d y(t)$, где $d y(t)$ - собственная функция, соответствуюшая достаточно большим значениям спектра.

Поэтому точка $(0,0)$ будет неустойчивой относительно $d I(t)$, что и приводит к неустойчивости указанных вьше решений уравнения (1.23).

\section{$\S$ 5. Устойчивость стационарных решений и бесконечно-мерный аналог теории КАМ}

В этом параграфе будут сформулированы теорема 1.10 и ее следствие 1.4 , согласно которым, при некоторых общих предположениях на функцию $\varphi(q)$ и параметры $m_{n}$, стационарное решение уравнения (1.1), имеющее вид

$$
q^{0}=\left(q_{n}^{0}(t)\right), \quad q_{n}^{0}(t)=q_{n}^{0} \equiv \eta+n a,
$$

в котором

$$
\varphi(\eta)=0
$$

а $\eta$ - константа, не зависящая от $n$ и $t$, будет устойчиво по Ляпунову относительно изменения начальных данных в любом из трех фазовых пространств $\Omega, \Omega_{\infty}, \Omega_{\infty}^{*}$, введенных в $\S 2$. Это означает, что малое изменение начальных данных по норме в соответствующем фазовом пространстве приводит к малому изменению координат $q_{n}(t)$ и импульсов $p_{n}(t)=m_{n} \frac{d q_{n}}{d t}$ соответствующей гамильтоновой системы $(\S 7$, гл. 1$)$ при всех $n \in \mathbb{Z}, t \in \mathbb{R}$. Утверждение теоремы 1.10 и следствия 1.4 справедливы для любого стационарного решения $\left(q_{n}(t)\right)$ уравнения $(1.1)$, в котором при произвольном $n \in \mathbb{Z}$ функция $q_{n}(t)$ принимает одно из значений вида $\eta+k a$, где $\varphi(\eta)=0$, a $k$ - целое число. В качестве следствия теоремы 1.10 мы находим, что в рассматриваемой ситуации имеет место бесконечно-мерньй аналог теории КАМ (теорема 1.11 и ее следствие 1.5): в любой окрестности стационарного решения (1.25) сушествуют семейства квази-периодических решений уравнения (1.1) с континуальным, счетным и любым конечным числом рационально независимых частот и торы произвольной конечной и бесконечной размерности, на которых решения уравнения (1.1) порождают квази-периодические движения.

Утверждения теорем 1.10 и 1.11 справедливы, если в качестве функции $\varphi(q)$ взять функцию $\varphi(q)=-h \sin \frac{2 \pi q}{a}$, где константа $h$ удовлетворяет неравенствам следствия 1.1 теоремы 1.10; они справедливы также для всех функций, близких к функции $\varphi(q)$ из следствия 1.1 (следствие 1.2).

Напомним, что, согласно определениям 1.2-1.4 $\S 2$, пространства $\Omega$ и $\Omega_{\infty}$ состоят из пар

$$
\left(q_{0}^{*}(t), q_{1}^{*}(t)\right) \in \Omega, \quad\left(q_{0}^{*}(t), q_{1}^{*}(t)\right) \in \Omega_{\infty}
$$


бесконечно-дифференцируемых и целых аналитических функций $q_{0}^{*}(t), q_{1}^{*}(t)$, определенных на прямой $t \in \mathbb{R}$, а пространство $\Omega_{\infty}^{*}$ состоит из двух последовательностей чисел $\left(q_{n}^{*}\right)$ и $\left(v_{n}^{*}\right)$, где $n \in \mathbb{Z}$ :

$$
\left\{\left(q_{n}^{*}\right),\left(v_{n}^{*}\right)\right\} \in \Omega_{\infty}^{*}
$$

При этом в силу теорем 1.3 и $1.5 \S 2$ любое решение $\left(q_{n}(t)\right)$ уравнения $(1.1)$ однозначно определяется начальными данными из $\Omega$ и $\Omega_{\infty}$ так, что

$$
q_{0}(t)=q_{0}^{*}(t), \quad q_{1}(t)=q_{1}^{*}(t)
$$

и начальными данными из $\Omega_{\infty}^{*}$ так, что для $n \in \mathbb{Z}$

$$
q_{n}\left(t^{*}\right)=q_{n}^{*}, \quad \frac{d q_{n}}{d t}\left(t^{*}\right)=v_{n}^{*}
$$

где $t^{*}$ - начальньй момент времени. Согласно (1.27)-(1.30) стационарное решение (1.25) однозначно определяется любым из следуюших трех видов начальных данных:

$$
\begin{gathered}
(\eta, \eta+a) \in \Omega, \quad q_{0}^{*}(t) \equiv \eta, \quad q_{1}^{*}(t) \equiv \eta+a ; \\
(\eta, \eta+a) \in \Omega_{\infty}, \quad q_{0}^{*}(t) \equiv \eta, \quad q_{1}^{*}(t) \equiv \eta+a ; \\
\{(\eta+n a),(0)\} \in \Omega_{\infty}^{*}, \quad q_{n}^{*}=\eta+n a, \quad v_{n}^{*}=0, \quad n \in \mathbb{Z} .
\end{gathered}
$$

Пусть $\kappa=\left(\kappa_{0}, \kappa_{1}, \ldots\right)$ - односторонняя последовательность, состоящая из заранее фиксированных положительных чисел $\kappa_{s}(s=0,1, \ldots)$. Далее будут рассматриваться возмущения начальных данных (1.31)-(1.33) следующего вида:

$$
\begin{aligned}
& \left(q_{0}^{*}(t), q_{1}^{*}(t)\right)=\left(\eta+\Delta_{0}(t), \eta+a+\Delta_{1}(t)\right) \in \Omega^{(\kappa)}\left(q^{0}\right), \\
& \left(q_{0}^{*}(t), q_{1}^{*}(t)\right)=\left(\eta+\Delta_{0}(t), \eta+a+\Delta_{1}(t)\right) \in \Omega_{\infty}^{(\kappa)}\left(q^{0}\right), \\
& \left\{\left(q_{n}^{*}\right),\left(v_{n}^{*}\right)\right\}=\left\{\left(\eta+n a+\Delta_{n}^{*}\right),\left(\widetilde{\Delta}_{n}^{*}\right)\right\} \in \Omega_{\infty}^{*(\kappa)}\left(q^{0}\right),
\end{aligned}
$$

где $q_{0}$ - стационарное решение, введенное в $(1.25), \Omega^{(\kappa)}\left(q^{0}\right), \Omega_{\infty}^{(\kappa)}\left(q^{0}\right), \Omega_{\infty}^{*(\kappa)}\left(q^{0}\right)$ - множества, введенные в определении $1.7 \S 2, \Delta_{0}(t)$ и $\Delta_{1}(t)$ - функции, определенные на прямой $t \in \mathbb{R}$, которые являются бесконечно-дифференшируемыми в равенстве (1.34) и цельми аналитическими в равенстве $(1.35)$, а $\Delta_{n}^{*}$ и $\widetilde{\Delta}_{n}^{*}$ - числа. В силу определения $1.7 \S 2$ и $(1.31),(1.32)$ соотношения (1.34) и (1.35) соответственно эквивалентны неравенствам:

$$
\|\Delta\|^{(k)} \leqslant \kappa_{k}
$$

где $k=0,1,2, \ldots ; \quad \Delta=\left(\Delta_{0}(t), \Delta_{1}(t)\right) \in \Omega$ для неравенства (1.34) и $\Delta=$ $\left(\Delta_{0}(t), \Delta_{1}(t)\right) \in \Omega_{\infty}$ для неравенства (1.35), а соотношение (1.36) эквивалентно неравенствам

$$
\rho^{(k)}\left(\left\{\left(q_{n}^{*}\right),\left(v_{n}^{*}\right)\right\},\{(\eta+n a),(0)\}\right) \leqslant \kappa_{k} ; \quad k=0,1,2, \ldots,
$$


где $\|\cdot\|^{(k)}$ - норма, введенная в определении $1.5 \S 2$, а $\rho^{(k)}(\cdot, \cdot)$ - расстояние в пространстве $\Omega_{\infty}^{*}$, введенное в определении $1.6 \oint 2$.

Теорема 1.10. Предположими, что $m_{n} \leqslant M$ при всех $n \in \mathbb{Z}$, существует такое $\delta_{0}>0$, что в области $|q-\eta| \leqslant \delta_{0}$ функция $\varphi(q)$ аналитична, и выполнень следующие условия:

1) $0<\frac{d \varphi}{d q}(\eta)<4$

2) $\frac{d \varphi}{d q}(\eta) \neq 2-2 \cos \frac{2 \pi s}{l}(s=0, \pm 1, \ldots, \pm l ; l=1,2,3,4)$;

3) $b_{0}(\lambda) \frac{d^{3} \varphi}{d q^{3}}(\eta)+b_{1}(\lambda)\left(\frac{d^{2} \varphi}{d q^{2}}(\eta)\right)^{2} \neq 0$, где

$b_{0}(\lambda)=-\frac{\lambda}{2(\lambda-\bar{\lambda})}$,

$b_{1}(\lambda)=\frac{\lambda^{2}}{(\lambda-\bar{\lambda})^{2}(1-\lambda)}-\frac{1}{(\lambda-\bar{\lambda})^{2}(1-\bar{\lambda})}+\frac{\lambda^{2}}{2(\lambda-\bar{\lambda})^{2}\left(\lambda^{2}-\lambda\right)}-\frac{1}{2(\lambda-\bar{\lambda})^{2}\left(\lambda^{2}-\bar{\lambda}\right)}$,

$\lambda$ - собственное значение матрицы

$$
\left(\begin{array}{cc}
1-\frac{d \varphi}{d q}(\eta) & 1 \\
-\frac{d \varphi}{d q}(\eta) & 1
\end{array}\right)
$$

удовлетворяющее условию $\operatorname{Im} \lambda>0, a \bar{\lambda}$ - число, комплексно-сопряхсенное $к \lambda$. Тогда существуют такие константы $m_{n}^{0}>0(n \in \mathbb{Z})$, зависящие только от последовательности к, числа $M$ и функиии $\varphi(q)$, что если при каком-либо $n_{0}$ $u|n| \geqslant n_{0}$ числа $m_{n}$ удовлетворяют условиям $m_{n} \leqslant m_{n}^{0}$, то стационарное решение $q^{0}=\left(q_{n}^{0}\right)$ из (1.25) устойчиво в следующем смысле: для любого $\varepsilon>0$ найдутся $\delta>0$ и натуральное число $k_{0}$, зависящие от $\varepsilon$, такие, что если вьполнено какое-либо из трех условий

а) соотношение (1.34) $u\left\|\left(\Delta_{0}(t), \Delta_{1}(t)\right)\right\|^{\left(k_{0}\right)} \leqslant \delta$,

б) соотношение $(1.35) u\left\|\left(\Delta_{0}(t), \Delta_{1}(t)\right)\right\|^{\left(k_{0}\right)} \leqslant \delta$,

в) соотношение (1.36) и $\rho^{\left(k_{0}\right)}\left(\left\{\left(q_{n}^{*}\right),\left(v_{n}^{*}\right)\right\},\{(\eta+n a),(0)\}\right) \leqslant \delta$,

то решение $\left(q_{n}(t)\right)$ уравнения (1.1) с начальнымм данными (1.34)-(1.36) при всех $n \in \mathbb{Z} u t \in \mathbb{R}$ удовлетворяет неравенству

$$
\left|q_{n}(t)-q_{n}^{0}\right| \leqslant \varepsilon
$$

Доказательство теоремы 1.10 будет проведено в следующем параграфе. Здесь же мы сформулируем и докажем следствия 1.1-1.4 из теоремы 1.10 , теорему 1.11 и ее следствие 1.5 .

СлЕДСТВИЕ 1.1. Если в условии теоремы 1.10 функция $\varphi(q)=-h \sin \frac{2 \pi q}{a}$, $0<h<\frac{2 a}{\pi}, h \neq \frac{a}{\pi}\left(1-\cos \frac{2 \pi s}{l}\right)(s=0, \pm 1, \ldots, \pm l ; l=1,2,3,4)$, а число $\eta=-\frac{a}{2}$, то справедливо утверждение теоремы 1.10 . 
ДокАЗАТЕльство. Достаточно проверить справедливость условия 3) теоремы 1.10, так как условия 1) и 2) вытекают непосредственно. В силу формулировки следствия 1.1 вьполнены соотношения $\frac{d^{2} \varphi}{d q^{2}}(\eta)=0, \frac{d^{3} \varphi}{d q^{3}}(\eta) \neq 0, \lambda \neq \bar{\lambda}$, которые и приводят к справедливости условия 3 ).

СлЕДСТВИЕ 1.2. Утверждение теоремы 1.10 справедливо для всех функиий $\varphi(q)$, близких вместе с первыми тремя производными к функции $\varphi(q)$ из формулировки следствия 1.1.

СлЕДСТВИЕ 1.3. Утверждение теоремы 1.10 выполнено в тех случаях, когда возмущения $\Delta_{\nu}(t)(\nu=0,1)$ в (1.34) и (1.35) представляются в виде функций

$$
\Delta_{\nu}(t)=\int_{|\omega| \leqslant N} h_{\nu}(\omega) \sin (u(\omega)+\omega t) d \omega, \quad \Delta_{\nu}(t)=\sum_{k} h_{\nu}^{(k)} \sin \left(u_{k}+\omega_{k} t\right)
$$

где $h_{\nu}(\omega)$ - абсолютно интегрируемая вещественная функиия, $u(\omega)$ - измеримая по Лебегу вещественная функчия, $u_{k}, \omega_{k}$ - вещественные числа, $\left|h_{\nu}(\omega)\right| \leqslant \gamma$, $\sum_{k}\left|h_{\nu}^{(k)}\right| \leqslant \gamma,\left|\omega_{k}\right| \leqslant N$, индекс $k$ пробегает конечное или счетное мнохсество, $\gamma$ - достаточно малая положительная константа, не зависящая от $\nu$ u.

ДоКАЗАТЕльСТВо. В силу (1.39) и определения 1.7 (§2) для некоторой последовательности чисел $\kappa=\left(\kappa_{0}, \kappa_{1}, \ldots\right)$ функции $\Delta_{\nu}(t)(\nu=0,1)$ на прямой $t \in \mathbb{R}$ удовлетворяют одному из соотношений $(1.34),(1.35)$, и поэтому к ним применима теорема 1.10. При фиксированном $\varepsilon>0$, выбирая достаточно малое $\gamma>0$, согласно (1.39) мы удовлетворим одному из соотношений а), б) в условии теоремы 1.10 , а это и приводит к справедливости утверждения теоремы 1.10. Следствие 1.3 доказано.

СлЕДСТВИЕ 1.4. Предположим, что при всех $n \in \mathbb{Z}$ параметры $m_{n}$ в уравнении (1.1) удовлетворяют неравенствам $m_{n} \leqslant$. Тогда существует такая константа $K$, зависящая только от числа $M$ и функиии $\varphi(q)$, что решения $\left(q_{n}(t)\right)$ уравнения (1.1), найденные в теореме 1.10 и удовлетворяющие неравенствам $\left|q_{n}(t)-q_{n}^{0}\right| \leqslant \varepsilon(n \in \mathbb{Z})$, при всех $n \in \mathbb{Z}, t \in \mathbb{R}$ также удовлетворяют неравенствам $\left|p_{n}(t)\right| \leqslant K \varepsilon$, где $p_{n}(t)=m_{n} \frac{d q_{n}}{d t}(t)$.

ДокАЗАТЕльСтво. Из вида уравнения (1.1) и (1.25), (1.26) следует равенство

$$
\frac{d p_{n}}{d t}(t)=\varphi\left(q_{n}(t)\right)-\varphi\left(q_{n}^{0}\right)+\left(q_{n+1}(t)-q_{n+1}^{0}\right)-2\left(q_{n}(t)-q_{n}^{0}\right)+\left(q_{n-1}(t)-q_{n-1}^{0}\right),
$$

которое приводит к неравенству

$$
\left|\frac{d p_{n}}{d t}(t)\right| \leqslant \varepsilon\left(4+\max _{0 \leqslant q \leqslant a}\left|\frac{d \varphi}{d q}(q)\right|\right) .
$$

Поэтому утверждение следствия 1.4 вытекает из формулы Тейлора для функции $m_{n} q_{n}(t)$ :

$$
m_{n} q_{n}(t+1)=m_{n} q_{n}(t)+p_{n}(t)+\frac{1}{2} \frac{d p_{n}}{d t}(\Theta),
$$

где $t \leqslant \Theta \leqslant t+1$. Следствие 1.4 доказано. 
ТЕорема 1.11 (бесконечно-мерньй аналог теории КАМ). Предположим, что выполняются все условия теоремы 1.10. Тогда существуют такие константь $m_{n}^{0}>0(n \in \mathbb{Z})$, что если при каком-либо $n_{0}$ u $|n| \geqslant n_{0}$ числа $m_{n}$ удовлетворяют неравенствам $m_{n} \leqslant m_{n}^{0}$, то при любом $\varepsilon>0$ существуют такие бесконечные множсества квази-периодических решений $\left(q_{n}(t)\right)$ уравнения $(1.1)$ с континуальнымм, счетным и любым конечным количеством рачионально независимых частот, что при всех $n \in \mathbb{Z}, t \in \mathbb{R}$ выполняется неравенство

$$
\left|q_{n}(t)-q_{n}^{0}\right| \leqslant \varepsilon
$$

ДокАЗАТЕЛЬство. Определим квази-периодические функции $q_{0}^{*}(t)$ и $q_{1}^{*}(t)$ в $(1.34)$ и (1.35) с указанным в теореме 1.11 количеством рационально независимых частот, используя функции $\Delta_{0}(t)$ и $\Delta_{1}(t)$, которые введены в следствии 1.3 с помощью равенств (1.39). Пусть $\delta_{0}-$ константа, введенная в условии теоремы 1.10 . Согласно утверждению теоремы 1.10 и следствию 1.3 , для любого положительного $\varepsilon \leqslant \delta_{0}$ существуют такие константы $m_{n}^{0}>0$, что если при каком-либо $n_{0}$ и $|n| \geqslant n_{0}$ справедливы неравенства $m_{n} \leqslant m_{n}^{0}$, то решение $\left(q_{n}(t)\right)$ уравнения (1.1) с начальными данными $\left(q_{0}^{*}(t), q_{1}^{*}(t)\right)$ при всех $n \in \mathbb{Z}, t \in \mathbb{R}$ удовлетворяет неравенствам (1.40). Так как при этом функции $q_{0}(t)$ и $q_{1}(t)$, входяшие в решение $\left(q_{n}(t)\right)$, имеют вид $q_{0}(t)=q_{0}^{*}(t)$, $q_{1}(t)=q_{1}^{*}(t)$, а согласно условию теоремы 1.10 функция $\varphi(q)$ имеет период $a$ по $q$ и аналитична в области $|q-\eta| \leqslant \delta_{0}$, то в силу определений 7$\left.)-10\right)$ (см. обшие обозначения и определения) из вида уравнения (1.1), из теоремы $1.3 \S 2$, из равенства (1.25) и неравенств (1.40) и $\varepsilon \leqslant \delta_{0}$ следует, что при всех $n \in \mathbb{Z}$ функции $q_{n}(t)$ будут квази-периодическими с тем же набором частот, что и функции $\Delta_{0}(t), \Delta_{1}(t)$. Теорема 1.11 доказана.

СЛЕДСТВИЕ 1.5. Если выполнены условия теоремы 1.10 и при $|n| \geqslant n_{0}$ спра-

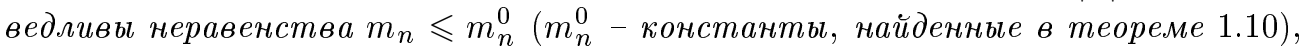
то для любого в $>0$ в области $\Gamma_{\varepsilon}=\left\{\vec{q}=\left(q_{n}\right):\left|q_{n}-q_{n}^{0}\right| \leqslant \varepsilon, n \in \mathbb{Z}\right\}$ существуют инвариантные множества, составленные из решений уравнения (1.1), которые представляют собой торы любой конечной размерности, счетно-мерные торь и существенное подмножество тора континуальной размерности (n. n. 12), 13), общие обозначения и определения), а решения уравнения (1.1), расположенные на этих торах, порождают на них квази-периодические движения (n. 14), общие обозначения и определения).

ДокАЗАТЕльство. Если в выражениях (1.39) для функций $\Delta_{\nu}(t)$, введенных в следствии 1.3 , при фиксированных числах $h_{\nu}^{(k)}$ и функции $h_{\nu}(\omega)$ менять произвольным образом числа $u_{k}$ в области $0 \leqslant u_{k}<2 \pi$ и измеримую функцию $u(\omega)$ в области $0 \leqslant u(\omega)<2 \pi$, то в силу теоремы 1.11 при достаточно малом $\gamma>0$ множество соответствуюших решений уравнения (1.1) лежит в области $\Gamma_{\varepsilon}$, будет заполнять конечно-мерньй или счетно-мерньй тор, если, соответственно, множество чисел $k-$ конечное или счетное, а функции $\Delta_{\nu}(t)(\nu=0,1)$ определяются с помошњю второго равенства в (1.39), и будет заполнять существенное подмножество тора континуальной размерности, если функщии $\Delta_{\nu}(t)$ определяются с помошюю первого равенства 
в (1.39). При этом, согласно теореме 1.11 и определениям 11)-14) (общие обозначения и определения), решения уравнения (1.1) порождают на соответствующем торе квази-периодические движения: $\widetilde{u}_{k}(t)=u_{k}+\omega_{k} t(\bmod 2 \pi)$ в случае конечно-мерного или счетно-мерного тора, и $\widetilde{u}(t)=u(\omega)+\omega t(\bmod 2 \pi)$ в случае тора континуальной размерности. Следствие 1.5 доказано.

\section{§6. Доказательство теоремы 1.10}

Для любого натурального числа $s$ обозначим через

$$
\varphi_{s}=\sup _{\substack{1 \leqslant k \leqslant s \\ 0 \leqslant q \leqslant a}}\left|\frac{d^{k} \varphi}{d q^{k}}(q)\right|
$$

Нам потребуются четыре леммы.

ЛЕмма 1.2. Пусть $G(t)=\varphi(q(t)), s$ - натуральное число. Тогда выражсение для производной $\frac{d^{s} G}{d t^{s}}(t)$ есть сумма не более чем s! одночленов, каждый из которых есть произведение какой-либо производной $\frac{d^{k} \varphi_{k}}{d q^{k}}(q)(1 \leqslant k \leqslant s)$ на произведение производных по $t$ порядка, не превосходящего $s$, от функиии $q(t)$ в количестве, не превосходящем $s$.

ДокАЗАТЕльство. Проведем индукцию по $s$. При $s=1$ утверждение леммы 1.2 очевидно. Предположим, что утверждение справедливо для некоторого $s \geqslant 1$, и вычислим производную от выражения $\frac{d^{s} G}{d t^{s}}(t)$ по $t$. При вычислении производной от каждого одночлена мы получим не более чем $s+1$ одночленов, каждый из которых есть произведение какой-либо производной $\frac{d^{k} \varphi}{d q^{k}}(q)(k \leqslant s+1)$ на произведение производных по $t$ порядка, не превосходяшего $s+1$, от функции $q(t)$ в количестве, не превосходящем $s+1$. Лемма 1.2 доказана

ЛЕмма 1.3. Предположим, что бесконечно-дифферениируемая функиия $q(t)$ при всех $t \in \mathbb{R}$ и натуральных s удовлетворяет условиям

$$
\left|\frac{d^{s} q}{d t^{s}}(t)\right| \leqslant c^{(s)}, \quad 1 \leqslant c^{(s)} \leqslant c^{(s+1)},
$$

$a G(t)=\varphi(q(t))$. Тогда

$$
\left|\frac{d^{s} G}{d t^{s}}(t)\right| \leqslant \varphi_{s} s !\left(c^{(s)}\right)^{s} .
$$

Лемма 1.3 есть очевидное следствие леммы 1.2 .

ЛЕмма 1.4. Предположим, что для всех натуральных $s$ u $\nu=n, n-1$ функция $q_{\nu}(t)$ - бесконечно-дифференцируемая, $\left|\frac{d^{s} q_{\nu}}{d t^{s}}(t)\right| \leqslant c_{n}^{(s)}, m_{\nu} \leqslant M$, где $1 \leqslant c_{n}^{(s)} \leqslant c_{n}^{(s+1)}$, a $q_{n+1}(t)$ и $q_{n-2}(t)-$ функции, удовлетворяюшие уравнению (1.1) при заданных функциях $q_{n}(t)$ u $q_{n-1}(t)$. Тогда при $i=n+1, n-2$ имеем $\left|\frac{d^{s} q_{i}}{d t^{s}}(t)\right| \leqslant c_{n+1}^{(s)}$, zде $c_{n+1}^{(s)}=3 c_{n}^{(s)}+\varphi_{s} \cdot s !\left(c_{n}^{(s)}\right)^{s}+M c_{n}^{(s+2)}$. 
ДоКАЗАТЕльство. В силу (1.1) имеем следующие равенства:

$$
\begin{aligned}
& \frac{d^{s} q_{n+1}}{d t^{s}}=-\frac{d^{s} q_{n-1}}{d t^{s}}+2 \frac{d^{s} q_{n}}{d t^{s}}-\frac{d^{s} \varphi\left(q_{n}(t)\right)}{d t^{s}}+m_{n} \frac{d^{s+2} q_{n}}{d t^{s+2}} \\
& \frac{d^{s} q_{n-2}}{d t^{s}}=-\frac{d^{s} q_{n}}{d t^{s}}+2 \frac{d^{s} q_{n-1}}{d t^{s}}-\frac{d^{s} \varphi\left(q_{n-1}(t)\right)}{d t^{s}}+m_{n-1} \frac{d^{s+2} q_{n-1}}{d t^{s+2}}
\end{aligned}
$$

Из этих равенств следует, что при $i=n+1, n-2$

$$
\left|\frac{d^{s} q_{i}}{d t^{s}}(t)\right| \leqslant 3 c_{n}^{(s)}+M c_{n}^{(s+2)}+\max _{i=n, n-1}\left|\frac{d^{s} \varphi\left(q_{i}(t)\right)}{d t^{s}}\right| .
$$

Применяя теперь лемму 1.3, получим утверждение леммы 1.4. Лемма 1.4 доказана.

Лемма 1.5. Предположим, что при всех $n \in \mathbb{Z} m_{n} \leqslant M$, а начальные даннье решения $\left(q_{n}(t)\right)$ уравнения (1.1) удовлетворяют какому-либо из соотношений (1.34), (1.35), (1.36). Тогда существуют такие константы $m_{n}^{0}>0$, что если при каком-либо $n_{0}$ u $|n| \geqslant n_{0}$ выполнены неравенства $m_{n} \leqslant m_{n}^{0}$, то решение $q_{n}(t)$ удовлетворяет неравенству

$$
\sum_{n=-\infty}^{\infty} m_{n} \sup _{t \in \mathbb{R}}\left|\frac{d^{2} q_{n}}{d t^{2}}(t)\right| \leqslant c^{*}
$$

где $c^{*}$ - константа, не зависящая от начальных данных.

ДокАЗАТЕльство. Как было указано в $\S 5$, условия (1.34), (1.35), (1.36) эквивалентны условиям (1.37), (1.38) соответственно. Поэтому утверждение леммы 1.5 очевидно следует из определений 1.5 и $1.6 \S 2$ и леммы 1.4. Лемма 1.5 доказана.

Предположим, что $\left(q_{n}(t)\right)$ есть решение уравнения (1.1). Введем преобразование $A$ и две последовательности семейств преобразований $B_{k}^{\prime}(t), B_{k}^{\prime \prime}(t)(k=1,2, \ldots)$ плоскости $u, z$, зависяших от параметра $t$. Преобразование $A$ есть отображение, которое определяется с помошью равенства

$$
A:(u, z) \rightarrow\left(u^{\prime}, z^{\prime}\right)=\left(u+z^{\prime}, z-\varphi(u)\right)
$$

а $B_{k}^{\prime}(t)$ и $B_{k}^{\prime \prime}(t)$ - отображения, которые задаются с помошњю функций $q_{n}(t)$ :

$$
\begin{aligned}
& B_{k}^{\prime}(t):(u, z) \rightarrow\left(u_{k}^{\prime}(t), z_{k}^{\prime}(t)\right)=\left(u, z+m_{k} \frac{d^{2} q_{k}}{d t^{2}}(t)\right) \\
& B_{k}^{\prime \prime}(t):(u, z) \rightarrow\left(u_{k}^{\prime \prime}(t), z_{k}^{\prime \prime}(t)\right)=\left(u, z+m_{-k+1} \frac{d^{2} q_{-k+1}}{d t^{2}}(t)\right)
\end{aligned}
$$

Вводя функции

$$
\begin{array}{ll}
z_{n+1}^{\prime}(t)=q_{n+1}(t)-q_{n}(t) & (n=0,1,2, \ldots), \\
z_{n-1}^{\prime \prime}(t)=q_{n-1}(t)-q_{n}(t) & (n=1,0,-1,-2, \ldots),
\end{array}
$$


мы из уравнения (1.1) получим следующие равенства:

$$
\begin{array}{ll}
\left(q_{n+1}(t), z_{n+1}^{\prime}(t)\right)=B_{n}^{\prime}(t) \circ A\left(q_{n}(t), z_{n}^{\prime}(t)\right) & (n=1,2, \ldots), \\
\left(q_{n-1}(t), z_{n-1}^{\prime \prime}(t)\right)=B_{1-n}^{\prime \prime}(t) \circ A\left(q_{n}(t), z_{n}^{\prime \prime}(t)\right) & (n=0,-1,-2, \ldots),
\end{array}
$$

которые приводят к равенствам

$$
\begin{aligned}
& \text { (1.44) }\left(q_{k}(t), z_{k}^{\prime}(t)\right)=B_{k-1}^{\prime}(t) \circ A \circ B_{k-2}^{\prime}(t) \circ A \circ \cdots \circ B_{1}^{\prime}(t) \circ A\left(q_{1}(t), z_{1}^{\prime}(t)\right) \\
& (k=2,3, \ldots) \text {, } \\
& \text { (1.45) }\left(q_{r}(t), z_{r}^{\prime \prime}(t)\right)=B_{-r}^{\prime \prime}(t) \circ A \circ B_{-r-1}^{\prime \prime}(t) \circ A \circ \cdots \circ B_{1}^{\prime \prime}(t) \circ A\left(q_{0}(t), z_{0}^{\prime \prime}(t)\right) \text {, } \\
& (r=-1,-2, \ldots) \text {. }
\end{aligned}
$$

Введем две последовательности преобразований $A_{k}^{\prime}, A_{k}^{\prime \prime}(k=1,2, \ldots)$ плоскости $u$, z следуюшим образом:

(1.46) $\quad A_{k}^{\prime}:(u, z) \rightarrow\left(\widetilde{u}_{k}^{\prime}, \widetilde{z}_{k}^{\prime}\right)=A(k a+\eta+u, z+a)-(k a+\eta+a, a)$,

(1.47) $\quad A_{k}^{\prime \prime}:(u, z) \rightarrow\left(\widetilde{u}_{k}^{\prime \prime}, \widetilde{z}_{k}^{\prime \prime}\right)=A(-k a+\eta+a+u, z-a)-(-k a+\eta,-a)$,

где $\eta$ - величина, введенная в (1.25) и (1.26). В силу (1.41), (1.46) и (1.47) преобразования $A_{k}^{\prime}, A_{k}^{\prime \prime}$ не зависят от $k$ и совпадают с преобразованием $\widetilde{A}$, имеюшим следуюший ВИД

$$
\widetilde{A}:(u, z) \rightarrow(\widetilde{u}, \widetilde{z})=(u+\widetilde{z}, z-\varphi(u+\eta)) .
$$

Лемма 1.6. Существуют такие константы $m_{n}^{0}>0$, зависящие только от числа $M$, последовательности $\kappa=\left(\kappa_{0}, \kappa_{1}, \ldots\right)$ и от функиии $\varphi(q)$, что если при каком-либо $n_{0} u|n| \geqslant n_{0}$ массь $m_{n}$ удовлетворяют условиям $m_{n} \leqslant m_{n}^{0}$, то для любого $\varepsilon>0$ найдутся такие $\delta>0$ и натуральное число $k_{0}$, что если выполнено какое-либо из соотношений (1.34)-(1.36), а при $\nu=0,1$ и $t \in \mathbb{R}$ функция $\Delta_{\nu}(t)$ удовлетворяет неравенствам

$$
\left|\Delta_{\nu}(t)\right| \leqslant \delta, \quad\left|\frac{d^{k} \Delta_{\nu}}{d t^{k}}(t)\right| \leqslant \delta \quad\left(k=1, \ldots, k_{0}\right),
$$

то при всех $t \in \mathbb{R}$ и цельх $k \geqslant 2$ функции $\widehat{q}_{k}^{\prime}(t), \widehat{z}_{k}^{\prime}(t), \widehat{q}_{k}^{\prime \prime}(t), \widehat{z}_{k}^{\prime \prime}(t)$, определеннье с помощью равенств

$$
\begin{aligned}
\left(\widehat{q}_{k}^{\prime}(t), \widehat{z}_{k}^{\prime}(t)\right) & =B_{k-1}^{\prime}(t) \circ \widetilde{A} \circ \cdots \circ B_{1}^{\prime}(t) \circ \widetilde{A}\left(\Delta_{1}(t), \Delta_{1}(t)-\Delta_{0}(t)\right), \\
\left(\widehat{q}_{k}^{\prime \prime}(t), \widehat{z}_{k}^{\prime \prime}(t)\right) & =B_{k-1}^{\prime \prime}(t) \circ \widetilde{A} \circ \cdots \circ B_{1}^{\prime \prime}(t) \circ \widetilde{A}\left(\Delta_{0}(t), \Delta_{0}(t)-\Delta_{1}(t)\right),
\end{aligned}
$$

удовлетворяют неравенствам

$$
\left|\widehat{q}_{k}^{\prime}(t)\right|+\left|\widehat{z}_{k}^{\prime}(t)\right| \leqslant \varepsilon, \quad\left|\widehat{q}_{k}^{\prime \prime}(t)\right|+\left|\widehat{z}_{k}^{\prime \prime}(t)\right| \leqslant \varepsilon .
$$


ДокАЗАТЕЛьство. Возьмем в качестве $m_{n}^{0}$ константы, найденные в лемме 1.5 , и покажем, что при выполнении условий теоремы 1.10 каждое из множеств преобразований $\widetilde{A}, B_{k}^{\prime}(t)(k=1,2, \ldots)$ и $\widetilde{A}, B_{k}^{\prime \prime}(t)(k=1,2, \ldots)$ в некоторой окрестности точки $(0,0)$ удовлетворяет всем условиям теоремы 2.6 ( 55, гл. 2$)$, которые в нашем случае сводятся к следуюшим утверждениям:

I) преобразование $\widetilde{A}$ определено в некоторой комплексной окрестности

$$
V_{\varepsilon_{0}}=\left\{u, z: \max (|u|,|z|) \leqslant \varepsilon_{0}\right\},
$$

где $\varepsilon_{0}>0$, и $(0,0)$ - его неподвижная точка;

II) преобразование $\widetilde{A}$ сохраняет площадь, имеет в точке $(0,0)$ общий эллиптический тип и в его нормальной форме Биркгофа $[51, \S 21]$

$$
\alpha^{\prime}=\alpha+\omega_{0}+\omega_{1} r+\cdots+\omega_{k} r^{k}+\cdots, \quad r^{\prime}=r,
$$

$\left(\alpha, r\right.$ - полярные координаты; $\alpha$ - угол,$r$ - радиус), коэффициент $\omega_{1} \neq 0$;

III) коэффициент $\omega_{0}$, входящий в $(1.50)$ и в выражения для собственных значений $\lambda=e^{i \omega_{0}}, \bar{\lambda}=e^{-i \omega_{0}}$, линейной части преобразования $\widetilde{A}$ в точке $(0,0)$, удовлетворяет неравенствам $\omega_{0} \neq 2 \pi \frac{s}{l}(s=0, \pm 1, \ldots, \pm l ; l=1,2,3,4)$;

IV) при всех $t$ и натуральных $k B_{k}^{\prime}(t)$ и $B_{k}^{\prime \prime}(t)$ - гомеоморфизмы области $\operatorname{Re} V_{\varepsilon_{0}}$ $\left(\operatorname{Re} V_{\varepsilon_{0}}-\right.$ вешественная часть $\left.V_{\varepsilon_{0}}\right)$, имеющие вид

$$
\begin{aligned}
& B_{k}^{\prime}(t):(u, z) \rightarrow\left(u_{k}^{\prime}(t), z_{k}^{\prime}(t)\right)=\left(u+b_{1 k}^{\prime}(u, z, t), z+b_{2 k}^{\prime}(u, z, t)\right), \\
& B_{k}^{\prime \prime}(t):(u, z) \rightarrow\left(u_{k}^{\prime \prime}(t), z_{k}^{\prime \prime}(t)\right)=\left(u+b_{1 k}^{\prime \prime}(u, z, t), z+b_{2 k}^{\prime \prime}(u, z, t)\right),
\end{aligned}
$$

и при этом

$$
\sum_{k=1}^{\infty} \sup _{(u, z) \in \operatorname{Re} V_{\varepsilon_{0}}}\left(\left|b_{1 k}^{\prime}(u, z, t)\right|+\left|b_{2 k}^{\prime}(u, z, t)\right|+\left|b_{1 k}^{\prime \prime}(u, z, t)\right|+\left|b_{2 k}^{\prime \prime}(u, z, t)\right|\right)<\infty .
$$

Утверждение I) следует из (1.48) и условия теоремы (1.10), согласно которому функция $\varphi(q)$ аналитична в области $|q-\eta| \leqslant \delta_{0}$, а утверждение IV) - из (1.42), (1.43) и леммы 1.5. В силу (1.48) преобразование $\widetilde{A}$ сохраняет плошадь, а согласно условию 1$)$ теоремы 1.10 след $\mathrm{Sp} d \widetilde{A}$ матрицы $d \widetilde{A}(0,0)$, задающей линейную часть $\widetilde{A}$ в точке $(0,0)$, удовлетворяет неравенству $|\operatorname{Sp} d \widetilde{A}|<2$. Поэтому $\widetilde{A}$ в точке $(0,0)$ имеет эллиптический тип. Далее, из условия 2) теоремы 1.10 следует утверждение III), тогда как утверждение II) вьводится из условия 3) теоремы 1.10 в результате приведения преобразования $\widetilde{A}$ к нормальной форме Биркгофа в неподвижной точке $(0,0)$. Таким образом, утверждения I)-IV) справедливы, и, следовательно, два множества преобразований $\widetilde{A}, B_{k}^{\prime}(t)$ и $\widetilde{A}, B_{k}^{\prime \prime}(t)(k=1,2, \ldots)$ удовлетворяют условию теоремы 2.6 из $\S 5$ гл. 2. Применяя эту теорему, получим следующее утверждение: для любого $\varepsilon>0$ найдется $\widehat{\delta}>0$, удовлетворяющее условию $\widehat{\delta} \leqslant \varepsilon$, и натуральные числа $\widehat{n}^{\prime}, \widehat{n}^{\prime \prime}$ такие, что если $|u| \leqslant \widehat{\delta},|z| \leqslant \widehat{\delta}$, то для любых натуральных чисел $k^{\prime} \geqslant \widehat{n}^{\prime}, k^{\prime \prime} \geqslant \widehat{n}^{\prime \prime}$ при всех $t \in \mathbb{R}$ справедливы неравенства

$$
\begin{array}{r}
\left|B_{k^{\prime}}^{\prime}(t) \circ \widetilde{A} \circ \cdots \circ B_{\widehat{n}^{\prime}}^{\prime}(t) \circ \widetilde{A}(u, z)\right| \leqslant \varepsilon, \\
\left|B_{k^{\prime \prime}}^{\prime \prime}(t) \circ \widetilde{A} \circ \cdots \circ B_{\widehat{n}^{\prime \prime}}^{\prime \prime}(t) \circ \widetilde{A}(u, z)\right| \leqslant \varepsilon
\end{array}
$$


(здесь $|(u, z)|=|u|+|z|)$. Согласно двум последним неравенствам, для доказательства леммы 1.6 достаточно показать, что для любого $\widehat{\delta}>0$ найдутся такое $\delta>0$ и натуральное число $k_{0}$, что если при $\nu=0,1$ и $k=1,2, \ldots, k_{0}$ для всех $t \in \mathbb{R}$ вьполняются неравенства (1.49), то для всех $t \in \mathbb{R}$ и всех целых чисел $s$ и $r$, удовлетворяющих условиям $1 \leqslant s \leqslant \widehat{n}^{\prime}-1,1 \leqslant r \leqslant \widehat{n}^{\prime \prime}-1$, справедливы неравенства

$$
\begin{aligned}
\left|B_{s}^{\prime}(t) \circ \widetilde{A} \circ \cdots \circ B_{1}^{\prime}(t) \circ \widetilde{A}\left(\Delta_{1}(t), \Delta_{1}(t)-\Delta_{0}(t)\right)\right| \leqslant \widehat{\delta} \\
\left|B_{r}^{\prime \prime}(t) \circ \widetilde{A} \circ \cdots \circ B_{1}^{\prime \prime}(t) \circ \widetilde{A}\left(\Delta_{0}(t), \Delta_{0}(t)-\Delta_{1}(t)\right)\right| \leqslant \widehat{\delta} .
\end{aligned}
$$

Однако эти два последние неравенства и сушествование указанных выше чисел $\delta$ и $k_{0}$ очевидно следуют из определений преобразований $\widetilde{A}, B_{k}^{\prime}(t), B_{k}^{\prime \prime}(t)$ и леммы 1.4 . Лемма 1.6. доказана.

В силу определений 1.5, 1.6 (§ 2), если выполнено какое-либо из двух неравенств

$$
\|\left(\Delta_{0}(t), \Delta_{1}(t) \|^{\left(k_{0}\right)} \leqslant \delta, \quad \rho^{\left(k_{0}\right)}\left(\left\{\left(q_{n}^{*}\right),\left(v_{n}^{*}\right)\right\},\{(\eta+n a),(0)\}\right) \leqslant \delta,\right.
$$

то вьполнены неравенства (1.49). Поэтому, подставляя в равенства (1.44), (1.45) вместо преобразования $A$ его выражения через преобразование $\widetilde{A}=A_{k}^{\prime}=A_{k}^{\prime \prime}$, найденные с помошшю равенств (1.46), (1.47), мы вьводим утверждение теоремы 1.10 из леммы 1.6. Теорема 1.10 доказана.

\section{$\S$ 7. Гамильтонова форма уравнения с потенциалом Френкеля-Конторовой}

Рассмотрим функцию $f(q)$, удовлетворяющую равенству

$$
\varphi(q)=-\frac{d f}{d q}(q)
$$

и введем понятие гамильтониана уравнения (1.1).

ОПРЕДЕЛЕНИЕ 1.12. Гамильтонианом уравнения (1.1) называется формальньй ряд $H$ следуюшего вида:

$$
H=\sum_{n \in \mathbb{Z}}\left(\frac{p_{n}^{2}}{2 m_{n}}+f\left(q_{n}\right)+\frac{1}{2}\left(q_{n+1}-q_{n}-\alpha\right)^{2}\right)
$$

где $p_{n}=m_{n} v_{n}$-импульс точки $P_{n}$ (см. введение), а $\alpha$-константа, не зависящая от $n$.

Смысл этого определения, состоит в том, что оно приводит к следующему понятию.

ОПРЕДЕЛЕНИЕ 1.13. Введем семейство функций $H_{n_{-}, n_{+}}$, зависящих от цельх параметров $n_{-}$и $n_{+}$, удовлетворяюших условию $n_{-}+1<n_{+}$, которое порождено гамильтонианом $H$ :

$$
H_{n_{-}, n_{+}}=\frac{1}{2}\left(q_{n_{-}+1}-q_{n_{-}}-\alpha\right)^{2}+\sum_{n_{-}<n_{<n_{+}}}\left(\frac{p_{n}^{2}}{2 m_{n}}+f\left(q_{n}\right)+\frac{1}{2}\left(q_{n+1}-q_{n}-\alpha\right)^{2}\right)
$$


ЗАмечАнИЕ 1.3 . Каждую функцию $H_{n_{-}, n_{+}}$мы будем рассматривать как функцию независимых переменных $q_{n_{-+1}}, p_{n_{-}+1}, \ldots, q_{n_{+-1}}, p_{n_{+-1}}$, которая дополнительно зависит от параметров $q_{n_{-}}, q_{n_{+}}$.

ЗАмечАнИЕ 1.4 . Функщия $H_{n_{-}, n_{+}}$является гамильтонианом системы частиц $P_{k}$ $\left(n_{-} \leqslant k \leqslant n_{+}\right)$, в которой заданы траектории $q_{n_{-}}(t)$ и $q_{n_{+}}(t)$ частиц $P_{n_{-}}$и $P_{n_{+}}$.

ПРЕДЛОЖЕНИЕ 1.1. Если $n_{-}<n<n_{+}$, то производная $\frac{\partial H_{n_{-}, n_{+}}}{\partial p_{n}}=\frac{p_{n}}{m_{n}}$ не зависит от чисел $n_{-}$и $n_{+}$, а если $n_{-}+1<n<n_{+}-1$, то производная $\frac{\partial H_{n_{-}, n_{+}}}{\partial q_{n}}=-\varphi\left(q_{n}\right)-\left(q_{n+1}-2 q_{n}+q_{n-1}\right)$ не зависит от чисел $n_{-}$u $n_{+}$.

Предложение 1.1 и равенства (1.52), (1.53) дают основание ввести производные гамильтониана $H$ по переменньм $q_{n}, p_{n}$.

ОПРЕДЕЛЕНИЕ 1.14. Определим производные $\frac{\partial H}{\partial p_{n}}, \frac{\partial H}{\partial q_{n}}$ гамильтониана $H$ следующим образом:

$$
\begin{aligned}
& \frac{\partial H}{\partial p_{n}}=\frac{\partial H_{n_{-}, n_{+}}}{\partial p_{n}}, \text { где } n_{-}<n<n_{+} \\
& \frac{\partial H}{\partial q_{n}}=\frac{\partial H_{n_{-}, n_{+}}}{\partial q_{n}}, \text { где } n_{-}+1<n<n_{+}-1 .
\end{aligned}
$$

В силу определения 1.14 и предложения 1.1 уравнение (1.1) эквивалентно следующей бесконечно-мерной системе Гамильтона:

$$
\frac{d q_{n}}{d t}=\frac{\partial H}{\partial p_{n}}, \quad \frac{d p_{n}}{d t}=-\frac{\partial H}{\partial q_{n}}, \quad n \in \mathbb{Z} .
$$

В основе теории конечно-мерных гамильтоновых систем лежит теорема об интегральном инварианте Пуанкаре-Картана [52], [12]. Эта теорема допускает обобщение на бесконечно-мерную систему Гамильтона (1.54). Для того, чтобы это обобшение сформулировать и доказать, нам потребуется следуюшая лемма.

ЛЕмма 1.7. Пусть $n_{-}$и $n_{+}-$иелье числа, $n_{-}+1<n_{+} ;$рассмотрим две произвольные бесконечно-дифференцируемые функиии $q_{n_{-}}^{*}(t), q_{n_{+}}^{*}(t)(t \in \mathbb{R})$ и множество, состоящее из $2\left(n_{+}-n_{-}-1\right)$ чисел $q_{n_{-+1}}^{0}, \ldots, q_{n_{+-1}}^{0}, p_{n_{-}+1}^{0}, \ldots, p_{n_{+-1}}^{0}$. Тогда существует единственное решение системы (1.54) такое, что в начальный момент времени $t^{0}$ имеют место равенства

$$
q_{n}\left(t^{0}\right)=q_{n}^{0}, \quad p_{n}\left(t^{0}\right)=p_{n}^{0}, \quad n_{-}<n<n_{+},
$$

и при всех $t \in \mathbb{R}$

$$
q_{n_{-}}(t)=q_{n_{-}}^{*}(t), \quad q_{n_{+}}(t)=q_{n_{+}}^{*}(t) .
$$


ДокАЗАТЕЛЬСТВО. Пусть $s=n_{+}-n_{-}-1$, а $\vec{m}^{(s)}=\left(m_{1}^{(s)}, \ldots, m_{s}^{(s)}\right)$ и $\vec{q}^{(s)}(t)=$ $\left(q_{1}^{(s)}(t), \ldots, q_{s}^{(s)}(t)\right)$ - векторы с координатами

$$
m_{k}^{(s)}=m_{n_{-}+k}, \quad q_{k}^{(s)}=q_{k}^{(s)}(t)=q_{n_{-}+k}(t) \quad(k=1, \ldots, s) .
$$

В силу (1.54) и определений (1.13), (1.14) вектор $\vec{q}^{(s)}(t)$ удовлетворяет неавтономной $2 s$-мерной (фазовое пространство имеет размерность $2 s$ ) системе обыкновенных дифференщиальных уравнений

$$
\left\{\begin{array}{c}
m_{1}^{(s)} \frac{d^{2} q_{1}^{(s)}}{d t^{2}}=\varphi\left(q_{1}^{(s)}\right)-2 q_{1}^{(s)}+q_{2}^{(s)}+q_{n_{-}}^{*}(t), \\
m_{2}^{(s)} \frac{d^{2} q_{2}^{(s)}}{d t^{2}}=\varphi\left(q_{2}^{(s)}\right)+q_{1}^{(s)}-2 q_{2}^{(s)}+q_{3}^{(s)}(t), \\
\ldots \ldots \ldots \ldots \ldots \ldots \ldots \cdots \cdots \cdots \cdots \cdots \cdots \cdots \cdots \cdots \cdots \cdots \cdots \cdots \cdots \cdots \cdots \cdots \cdots \\
m_{s-1}^{(s)} \frac{d^{2} q_{s-1}^{(s)}}{d t^{2}}=\varphi\left(q_{s-1}^{(s)}\right)+q_{s-2}^{(s)}-2 q_{s-1}^{(s)}+q_{s}^{(s)}(t), \\
m_{s}^{(s)} \frac{d^{2} q_{s}^{(s)}}{d t^{2}}=\varphi\left(q_{s}^{(s)}\right)+q_{s-1}^{(s)}-2 q_{s}^{(s)}+q_{n_{+}}^{*}(t),
\end{array}\right.
$$

если $s \geqslant 3$; в случае $s=2$ вектор $\vec{q}^{(s)}(t)$ удовлетворяет системе из двух уравнений

$$
\left\{\begin{array}{l}
m_{1}^{(s)} \frac{d^{2} q_{1}^{(s)}}{d t^{2}}=\varphi\left(q_{1}^{(s)}\right)-2 q_{1}^{(s)}+q_{2}^{(s)}+q_{n_{-}}^{*}(t), \\
m_{2}^{(s)} \frac{d^{2} q_{2}^{(s)}}{d t^{2}}=\varphi\left(q_{2}^{(s)}\right)+q_{1}^{(s)}-2 q_{2}^{(s)}+q_{n_{+}}^{*}(t),
\end{array}\right.
$$

а в случае $s=1$ он удовлетворяет уравнению

$$
m_{1}^{(s)} \frac{d^{2} q_{1}^{(s)}}{d t^{2}}=\varphi\left(q_{1}^{(s)}\right)-2 q_{1}^{(s)}+q_{n_{-}}^{*}(t)+q_{n_{+}}^{*}(t) .
$$

Во всех трех случаях, согласно классической теореме о сушествовании и единственности решений конечно-мерных систем обыкновенных дифференциальных уравнений, вектор $\vec{q}^{(s)}(t)$ однозначно определяется по начальным данньм

$$
q_{k}^{(s)}\left(t^{0}\right)=q_{n_{-}+k}^{0}, \quad \frac{d q_{k}^{(s)}}{d t}\left(t^{0}\right)=\frac{p_{n_{-}+k}^{0}}{m_{n_{-}+k}} \quad(k=1, \ldots, s) .
$$

Таким образом, в силу (1.56) уравнение (1.1) будет справедливо для всех $n$ в промежутке $n_{-}<n<n_{+}$. Далее, из вида уравнения (1.1) следует, что если при каком-либо $n$ функции $q_{n}(t)$ и $q_{n+1}(t)$ определены, то по ним однозначно восстанавливаются функции $q_{n-1}(t)$ и $q_{n+2}(t)$. Поэтому, в силу того, что функции $q_{n_{-+1}}(t)$ и $q_{n_{+}-1}(t)$ уже определены, а, согласно условию леммы 1.7 , функции $q_{n_{-}}(t)$ и $q_{n_{+}}(t)$ заранее заданы, то при всех $n \in \mathbb{Z}$ однозначно находятся функции $q_{n}(t)$, удовлетворяюшие уравнению (1.1) и системе (1.54). Лемма 1.7 доказана.

Согласно лемме 1.7 , для любых заданных целых чисел $n_{-}, n_{+}$, удовлетворяюших неравенству $n_{-}+1<n_{+}$, и для любых бесконечно-дифференцируемых функций $q_{n_{-}}^{*}(t), q_{n_{+}}^{*}(t)$ в расширенном фазовом пространстве $\left\{\left(q_{n}, p_{n}\right), t\right\}(n \in \mathbb{Z})$ системы (1.54) можно построить трубку $\Pi_{n_{-}, n_{+}}$, проходящую через две различные замкнутые кривые $\gamma_{1}$ и $\gamma_{2}$, которая состоит из таких решений $\left(q_{n}(t), p_{n}(t)\right)$ системы (1.54), что при всех $t \in \mathbb{R}$ имеют место равенства (1.55). 
Теорема 1.12. Справедливо следующее равенство:

$$
\oint_{\gamma_{1}} \sum_{n_{-}<n<n_{+}} p_{n} d q_{n}-H_{n_{-}, n_{+}} d t=\oint_{\gamma_{2}} \sum_{n_{-}<n<n_{+}} p_{n} d q_{n}-H_{n_{-}, n_{+}} d t .
$$

ДокАЗАТЕЛЬСТво. В конечно-мерном фазовом пространстве $q_{n_{-}+1}, p_{n_{-}+1}, \ldots$, $q_{n_{+}-1}, p_{n_{+}-1}$ рассмотрим неавтономную гамильтонову систему с функцией Гамильтона

$$
\begin{aligned}
H^{*} & =H^{*}\left(q_{n_{-}+1}, p_{n_{-}+1}, \ldots, q_{n_{+}-1}, p_{n_{+}-1}, t\right) \\
& =H_{n_{-}, n_{+}}\left(q_{n_{-}+1}, p_{n_{-}+1}, \ldots, q_{n_{+}-1}, p_{n_{+}-1} ; q_{n_{-}}^{*}(t), q_{n_{+}}^{*}(t)\right) .
\end{aligned}
$$

В силу указанного в этом параграфе замечания 1.4 эта система описывает движение частиц $P_{n_{-}+1}, \ldots, P_{n_{+}-1}$ при условии, что частицы $P_{n_{-}}$и $P_{n_{+}}$двигаются согласно равенствам (1.55). Поэтому из определения трубки $\Pi_{n_{-}, n_{+}}$ следует, что решения $\left(q_{n}(t), p_{n}(t)\right)(n \in \mathbb{Z})$, входяшие в нее, таковы, что вектор $\left(q_{n_{-}+1}(t), p_{n_{-}+1}(t), \ldots, q_{n_{+}-1}(t), p_{n_{+-1}}(t)\right)$ является решением гамильтоновой системы с функцией Гамильтона $H^{*}$. Применяя к этой конечно-мерной системе теорему об интегральном инварианте Пуанкаре-Картана, получим утверждение теоремы 1.12 . Теорема 1.12 доказана.

\section{§. Лагранжева форма уравнения с потенциалом Френкеля-Конторовой}

Так же как и в предыдущем параграфе, будем предполагать, что выполнено равенство (1.51).

ОпРЕДЕЛЕНИЕ 1.15. Лагранжианом уравнения (1.1) назьвается формальный ряд

$$
L=\sum_{n \in \mathbb{Z}}\left(\frac{m_{n} v_{n}^{2}}{2}-f\left(q_{n}\right)-\frac{1}{2}\left(q_{n+1}-q_{n}-\alpha\right)^{2}\right)
$$

где $\alpha$ - константа, не зависящая от $n$.

ОПРЕДЕЛЕНИЕ 1.16. Введем семейство функций $L_{n_{-}, n_{+}}$, зависяших от целочисленных параметров $n_{-}, n_{+}$удовлетворяюших условию $n_{-}+1<n<n_{+}$, которое порождено лагранжианом $L$ :

$$
L_{n_{-}, n_{+}}=-\frac{1}{2}\left(q_{n_{-}+1}-q_{n_{-}}-\alpha\right)^{2}+\sum_{n_{-}<n<n_{+}}\left(\frac{m_{n} v_{n}^{2}}{2}-f\left(q_{n}\right)-\frac{1}{2}\left(q_{n+1}-q_{n}-\alpha\right)^{2}\right) .
$$

ЗАмЕчАнИЕ 1.5. Функщия $L_{n_{-}, n_{+}}$будет рассматриваться как функция независимых переменных $q_{n_{-}+1}, v_{n_{-}+1}, \ldots q_{n_{+}-1}, v_{n_{+}-1}$, которая дополнительно зависит от параметров $q_{n_{-}}, q_{n_{+}}$.

ЗАмечАниЕ 1.6. Функщия $L_{n_{-}, n_{+}}$является лагранжианом системы частиц $P_{k}$ $\left(n_{-} \leqslant k \leqslant n_{+}\right)$, в которой заданы траектории $q_{n_{-}}(t), q_{n_{+}}(t)$ частищ $P_{n_{-}}, P_{n_{+}}$. 
ОпРЕДЕЛЕНиЕ 1.17. Введем производные $\frac{\partial L}{\partial q_{n}}, \frac{\partial L}{\partial v_{n}}$, лагранжиана $L$ следующим образом:

$$
\begin{aligned}
& \frac{\partial L}{\partial q_{n}}=\varphi\left(q_{n}\right)+q_{n+1}-2 q_{n}+q_{n-1}=\frac{\partial L_{n_{-}, n_{+}}}{\partial q_{n}}, \quad \text { где } n_{-}+1<n<n_{+}-1 ; \\
& \frac{\partial L}{\partial v_{n}}=m_{n} v_{n}=\frac{\partial L_{n_{-}, n_{+}}}{\partial q_{n}}, \quad \text { где } n_{-}<n<n_{+} \cdot
\end{aligned}
$$

Согласно определению 1.17, уравнение (1.1) эквивалентно следующей бесконечно-мерной системе уравнений Лагранжа:

$$
\frac{d}{d t}\left(\frac{\partial L}{\partial \dot{q}_{n}}\right)-\frac{\partial L}{\partial q_{n}}=0, \quad n \in \mathbb{Z},
$$

где $\dot{q}_{n}=v_{n}$.

Далее мы сформулируем и докажем обобшение принципа наименьшего действия Гамильтона для уравнения (1.1).

Пусть $n_{-}$и $n_{+}-$произвольные целые числа, удовлетворяющие неравенству $n_{-}+1<n_{+}$, а $t_{1}$ и $t_{2}$ - фиксированные моменты времени такие, что $t_{1}<t_{2}$. Рассмотрим произвольное решение $\left(q_{n}(t)\right)$ уравнения $(1.1)$, и пусть $x_{n}(t)=q_{n}(t)+h_{n}(t)$ $(n \in \mathbb{Z})$ - произвольная гладкая кривая в бесконечно-мерном пространстве, удовлетворяющая следующим условиям:

$$
\begin{aligned}
h_{n_{-}}(t)=h_{n_{+}}(t)=0, & t_{1} \leqslant t \leqslant t_{2}, \\
h_{n}\left(t_{1}\right)=h_{n}\left(t_{2}\right)=0, & n_{-}<n<n_{+} .
\end{aligned}
$$

Теорема 1.13. Решение $\left(q_{n}(t)\right)$ уравнения (1.1) есть әкстремаль функционала

$$
\int_{t_{1}}^{t_{2}} L_{n_{-}, n_{+}}\left(x_{n_{-}+1}, \dot{x}_{n_{-}+1}, \ldots, x_{n_{+}-1}, \dot{x}_{n_{+}-1} ; q_{n_{-}}(t), q_{n_{+}}(t)\right) d t .
$$

ДокАЗАТЕльство. Уравнение Эйлера-Лагранжа для функционала, указанного в теореме 1.13, при вьполнении равенств (1.60) и (1.61) приводит к справедливости уравнений (1.59) при $n_{-}<n<n_{+}$. Поэтому утверждение теоремы 1.13 следует из эквивалентности системы (1.59) и уравнения (1.1). Теорема 1.13 доказана.

\section{Глава 2.}

\section{Теория уравнения с нелинейностью Ферми-Паста-Улама}

Глава 2 посвящена уравнению

$$
m_{n} \frac{d^{2} x_{n}}{d t^{2}}=x_{n+1}-(2-c) x_{n}+x_{n-1}+\beta\left[\left(x_{n+1}-x_{n}\right)^{r}-\left(x_{n}-x_{n-1}\right)^{r}\right]
$$

где $n \in \mathbb{Z}, m_{n}>0, c \geqslant 0, \beta>0, r \geqslant 3, r$ - нечетное число, о котором говорилось во введении. 


\section{$\S 1$ Построение семейств периодических решений и решений, порождающих колебания конечной дискретной струны}

В этом параграфе исследуется частньй случай уравнения $(2.1)$, когда массы $m_{n}$ всех точек одинаковые и равны $m>0$, а константа $c=0$, и в результате мы имеем уравнение

(2.2) $m_{n} \frac{d^{2} x_{n}}{d t^{2}}=x_{n+1}+x_{n-1}-2 x_{n}+\beta\left[\left(x_{n+1}-x_{n}\right)^{r}-\left(x_{n}-x_{n-1}\right)^{r}\right], \quad n \in \mathbb{Z}$,

которое при $m=1, r=3$ есть уравнение Ферми-Паста-Улама [2]-[4]. Далее будет доказана теорема 2.1, в которой для любого нечетного числа $r \geqslant 3$ строятся семейства периодических решений уравнения (2.2), причем определение периодических решений уравнений (2.1) и (2.2) и амплитуды колебаний здесь такие же, как и для уравнения Френкеля-Конторовой (см. определение $1.10, \S 3$, гл. 1).

ТеОрема 2.1. Для любых вещественных чисел $q, p$ существует бесконечное семейство различных периодических решений $\left(x_{n}(t)\right)$ уравнения $(2.2)$, имеющих вид

$$
x_{n}(t)=q+p n+\xi_{n}(t), \quad n \in \mathbb{Z},
$$

таких, что выполнены следующие утверждения:

1) при всех $n \in \mathbb{Z} u t \in \mathbb{R}$

$$
\xi_{n}(t)=-\xi_{n+1}(t)
$$

2) амплитуда колебаний решений из этого семейства принимает любое значение межсду 0 и $\infty$.

ДокАЗАТЕЛЬСТВо. Подставляя (2.3) и (2.4) в (2.2), получим, что функция $\xi_{n}(t)$ удовлетворяет уравнению

$$
m_{n} \frac{d^{2} \xi_{n}}{d t^{2}}=-4 \xi_{n}-2 \beta \sum_{k=0}^{\frac{1}{2}(r-1)}\left(\begin{array}{c}
r \\
2 k
\end{array}\right) p^{2 k}\left(2 \xi_{n}\right)^{r-2 k}
$$

которое имеет потенциал

$$
U=U\left(\xi_{n}\right)=2 \xi_{n}^{2}+\beta \sum_{k=0}^{\frac{1}{2}(r-1)} \frac{\left(\begin{array}{c}
r \\
2 k
\end{array}\right)}{r+1-2 k} p^{2 k}\left(2 \xi_{n}\right)^{r+1-2 k}
$$

т.е. $m_{n} \frac{d^{2} \xi_{n}}{d t^{2}}=-\frac{\partial U}{\partial \xi_{n}}$

Согласно (2.6), $U\left(\xi_{n}\right)$ - четная функщия, и если $\xi_{n}>0$, то $\frac{\partial U}{\partial \xi_{n}}>0$. Поэтому для любого $\sigma>0$ сушествует периодическое решение уравнения (2.5), имеюшее амплитуду колебаний, равную $\sigma$. В силу нечетности числа $r$ правая часть уравнения $(2.5)$ есть нечетная функция относительно $\xi_{n}$, и поэтому любое решение уравнения (2.5) определяет решение (2.3) с условием (2.4) уравнения (2.2), что и доказывает утверждения 1 ) и 2) теоремы 2.1. Теорема 2.1 доказана. 
ПРЕДЛОЖЕНИЕ 2.1. Утверждение теоремы 2.1 и ее доказательство справедливь для любого натурального числа $r \geqslant 2$.

Рассмотрим дискретную струну, которая представляется как объединение точек $P_{n}(n \in \mathbb{Z})$, перемешающихся согласно уравнениям $(2.2)$ вдоль вертикальных прямых $-\infty<x_{n}<\infty$, проходяших через точки $\tau=n$ на горизонтальной прямой $-\infty<\tau<\infty$, и растяжимых отрезков, соединяюших соседние точки $P_{n}$ и $P_{n+1}$ при всех $n \in \mathbb{Z}$ (рис. 2.1). Для любого вещественного числа $\tau$ обозначим через $P_{\tau}$ точку, расположенную на дискретной струне и на вертикальной прямой, проходящей через точку $\tau$ на горизонтальной прямой, а через $x_{\tau}(t)$ - величину смешения точки $P_{\tau}$ в момент времени $t$ вдоль прямой, проходящей через точку $\tau$ на горизонтальной прямой (рис. 2.1).

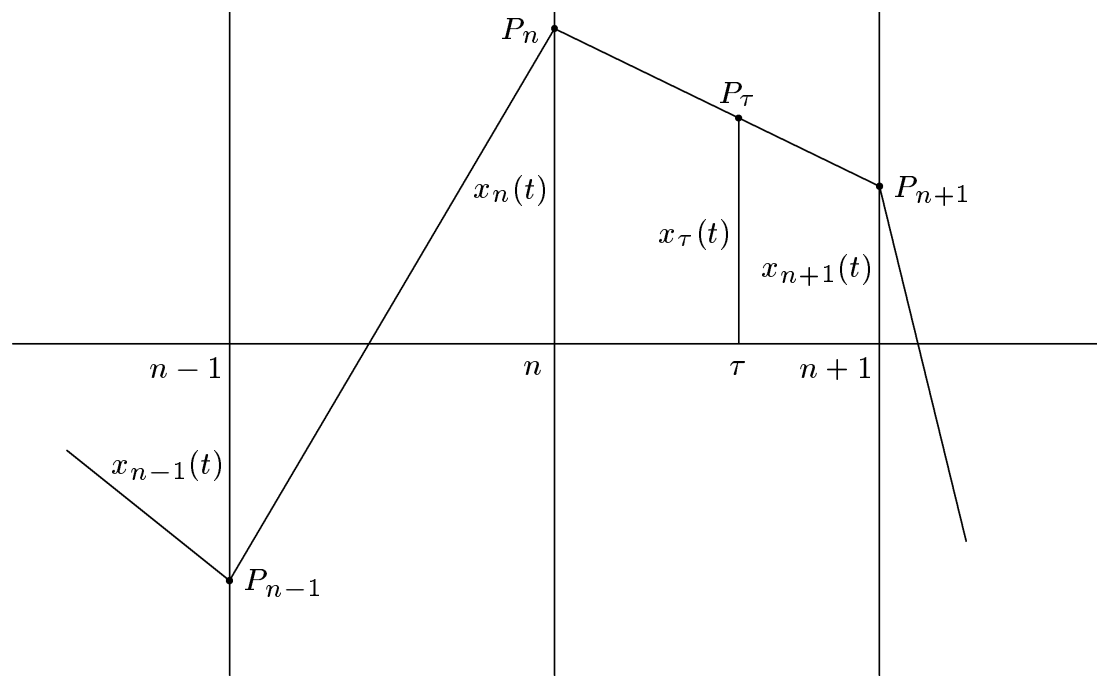

Рис. 2.1. Дискретная нелинейная струна

ОПРЕДЕЛЕНИЕ 2.1. Будем говорить, что решение $\left(x_{n}(t)\right)$ уравнения $(2.2)$ порождает колебания конечной дискретной струны на отрезке $\tau_{-} \leqslant \tau \leqslant \tau_{+}$, если существуют такие вещественные числа $\tau_{-}$и $\tau_{+}$, удовлетворяющие условию $\tau_{-}<\tau_{+}$, что при всех $t \in \mathbb{R}$ вьполняется равенство

$$
x_{\tau_{-}}(t)=x_{\tau_{+}}(t)=0 .
$$

СлЕДСТВИЕ 2.1. Пусть $\left(x_{n}(t)\right)=\left(\xi_{n}(t)\right)$ - периодическое решение уравнения (2.2), найденное в теореме 2.1 для $q=p=0 ; \tau_{-}=n_{-}+\frac{1}{2}, \tau_{+}=n_{+}+\frac{1}{2}$, где $n_{-}, n_{+}-$произвольные челье числа, $n_{-}<n_{+}$. Тогда решение $\left(x_{n}(t)\right)$ порожсдает колебания конечной дискретной струны на отрезке $\tau_{-} \leqslant \tau \leqslant \tau_{+}$.

ДокАЗАТЕльство. Для указанных в формулировке следствия 2.1 чисел $\tau_{-}$и $\tau_{+}$ равенство (2.7) вытекает из равенства (2.4) и определения дискретной струњы. Следствие 2.1 доказано.

В заключение этого параграффа сформулируем некоторые нерешенные задачи. 
ПроБлемА 2.1. Существуют ли какие-нибудь периодические решения уравнения (2.2), отличные от решений, найденных в теореме 2.1?

ПроБлема 2.2. Существуют ли какие-нибудь решения уравнения (2.2), отличные от решений, найденных в следствии 2.1, для которых существуют два или более различных отрезков $t_{-} \leqslant t \leqslant t_{+}$, на которых они порождают колебания конечной дискретной струны?

\section{$\S$ 2. Введение фазовых пространств и однозначная разрешимость уравнения в окрестности нулевого решения}

ОПРЕДЕЛЕНИЕ 2.2. Решение $x^{0}=\left(x_{n}^{0}(t)\right)$ уравнения $(2.1)$, в котором $x_{n}^{0}(t) \equiv 0$ при всех $n \in \mathbb{Z}$, будем назьвать нулевьм.

Имея ввиду в качестве главной задачи этой главы исследование устойчивости нулевого решения $(\S \S 3,4)$, мы в этом параграфе определим три фазовые пространства, состояшие из таких начальных данных, при которых решение уравнения (2.1), расположенное в некоторой окрестности нулевого решения, сушествует и единственно в классах бесконечно-дифференцируемых и аналитических функций. Отметим, что для стандартного фазового пространства это утверждение не имеет места (теорема 2.7, §6, гл. 2).

Прежде всего, докажем следуюшую лемму.

ЛЕмма 2.1. Предположим, что $\left(x_{n}(t)\right)$ - такое решение уравнения (2.1), что при всех $n \in \mathbb{Z}, t \in \mathbb{R}$ выполнено неравенство

$$
\left|x_{n}(t)\right|<\alpha
$$

əде

$$
\alpha=\frac{1}{2}\left(\frac{1}{r \beta}\right)^{1 /(r-1)} \text {. }
$$

Тогда функции $x_{n+1}(t), x_{n-2}(t)$ однозначно определяются через функции $x_{n}(t)$ $u x_{n-1}(t)$.

ДоКАЗАТЕЛЬСТво. Для произвольного $n \in \mathbb{Z}$ введем функции

$$
z_{n}(t)=x_{n}(t)-x_{n-1}(t), \quad \xi_{n}(t)=z_{n}(t)+\beta z_{n}^{r}(t) .
$$

Согласно (2.1) и $(2.10)$,

$\xi_{n+1}(t)=\xi_{n}(t)+m_{n} \frac{d^{2} x_{n}}{d t^{2}}(t)-c x_{n}(t), \quad \xi_{n-1}(t)=\xi_{n}(t)-m_{n} \frac{d^{2} x_{n-1}}{d t^{2}}(t)+c x_{n-1}(t)$,

и поэтому в силу $(2.10)$ функции $\xi_{n+1}(t)$ и $\xi_{n-1}(t)$ однозначно определяются через функции $x_{n}(t)$ и $x_{n-1}(t)$. 
Далее, при фиксированном $t$ второе равенство в (2.10) определяет $\xi_{n}(t)=\xi_{n}\left(z_{n}(t)\right)$ как функцию от $z_{n}(t)$, а из неравенств $(2.8),(2.9)$ и первого равенства в $(2.10)$ следует,что при фиксированном $t$ справедливо неравенство $\left|\frac{d \xi}{d z}\right| \neq 0$. Следовательно, при вьполнении неравенства (2.8) можно обратить второе равенство в (2.10) и однозначно выразить функцию $z_{n}(t)$ через $\xi_{n}(t)$. Таким образом, функции $z_{n+1}(t)$ и $z_{n-1}(t)$ однозначно определяются через функции $x_{n}(t)$ и $x_{n-1}(t)$, и поэтому такое же утверждение справедливо для функций $x_{n+1}(t)=x_{n}(t)+z_{n+1}(t)$ и $x_{n-2}(t)=x_{n-1}(t)-z_{n-1}(t)$. Лемма 2.1 доказана.

ЗАмЕчАнИЕ 2.1. Из теоремы 2.4 (следствие $2.2, \S 3$ ) вытекает, что для широкого класса уравнений (2.1) сушествует достаточно много решений $\left(x_{n}(t)\right)$, удовлетворяющих условию (2.8).

ОПРЕДЕЛЕНИЕ 2.3. Введем пространство П, состоящее из всевозможных пар $\left(x_{0}^{*}(t),\left(x_{1}^{*}(t)\right)\right.$, бесконечно-дифференцируемых функций $x_{0}^{*}(t), x_{1}^{*}(t)$, определенных на прямой $t \in \mathbb{R}$, для которых сушествует решение $\left(x_{n}(t)\right)$ уравнения $(2.1)$, удовлетворяющее равенствам

$$
x_{0}(t)=x_{0}^{*}(t), \quad x_{1}(t)=x_{1}^{*}(t),
$$

и при этом выполнено условие (2.8).

ОПРЕДЕЛЕНИЕ 2.4. Введем пространство П $\left(x_{0}^{*}(t),\left(x_{1}^{*}(t)\right)\right.$ целых аналитических функций $x_{0}^{*}(t), x_{1}^{*}(t)$, определенных на прямой $t \in \mathbb{R}$, для которых существует такое решение $\left(x_{n}(t)\right)$ уравнения $(2.1)$, что вьполнены условия (2.12) и (2.8).

ОПрЕДЕЛЕНИЕ 2.5. Введем пространство $\Pi_{\infty}^{*}$, состояшее из всевозможных пар $\left\{\left(x_{n}^{*}\right),\left(v_{n}^{*}\right)\right\}$ двух двусторонних последовательностей чисел $\left(x_{n}^{*}\right),\left(v_{n}^{*}\right)(n \in \mathbb{Z})$, для которых сушествует решение $\left(x_{n}(t)\right)$ уравнения (2.1), удовлетворяюшее $(2.8)$ и условиям

$$
x_{n}\left(t^{*}\right)=x_{n}^{*}, \quad \frac{d x_{n}}{d t}\left(t^{*}\right)=v_{n}^{*}, \quad n \in \mathbb{Z},
$$

где $t^{*}$ - начальньй момент времени, а при всех $n \in \mathbb{Z} x_{n}(t)$ - целая аналитическая функция на прямой $t \in \mathbb{R}$.

ТеОРема 2.2. В пространствах $\Pi$ и $\Pi_{\infty}$ уравнение (2.1) однозначно разрешимо, т.е. для любой парь функиий $x_{0}^{*}(t), x_{1}^{*}(t)$, удовлетворяющей условию $\left(x_{0}^{*}(t)\right.$, $\left.x_{1}^{*}(t)\right) \in \Pi$, существует единственное решение уравнения (2.1), для которого выполнены равенства (2.12).

Доказательство теоремы 2.2 следует из определений $2.3,2.4$ и леммы 2.1.

Теорема 2.3. Существует взаимно-однозначное соответствие $S: \Pi_{\infty}^{*} \rightarrow \Pi_{\infty}$ между пространствами $\Pi_{\infty}^{*} u \Pi_{\infty}, u$ для любого әлемента $\left\{\left(x_{n}^{*}\right),\left(v_{n}^{*}\right)\right\} \in \Pi_{\infty}^{*}$, существует единственное решение $\left(x_{n}(t)\right)$ уравнения (2.1) такое, что при всех $n \in \mathbb{Z} \quad x_{n}(t)$ - иелая аналитическая функиия $и$ в начальный момент времени $t^{*}$ выполнены равенства (2.13). 
ДокаЗАТЕЛЬСтво. Пусть $\left\{\left(x_{n}^{*}\right),\left(v_{n}^{*}\right)\right\} \in \Pi_{\infty}^{*}$. Сушествование решения $\left(x_{n}(t)\right)$, указанного в формулировке теоремы 2.3 , следует из определения 2.5 , а его единственность доказьвается точно так же, как доказьвалась единственность решения уравнения $(1.1)$ в теореме $1.1(\S 1$, гл. 1$)$. Построим теперь отображение $S$ и обратное к нему отображение $S^{-1}$. Полагаем

$$
S\left\{\left(x_{n}^{*}\right),\left(v_{n}^{*}\right)\right\}=\left(x_{0}(t), x_{1}(t)\right) \in \Pi_{\infty},
$$

где $x_{\nu}(t)(\nu=0,1)$ - составляюшие решения $\left(x_{n}(t)\right)$. Обратно, если $\left(x_{0}^{*}(t), x_{1}^{*}(t)\right) \in$ $\Pi_{\infty}$, то в силу определения 2.4 и теоремы 2.2 сушествует единственное решение $\left(x_{n}(t)\right)$ уравнения (2.1), для которого при всех $n \in \mathbb{Z} x_{n}(t)$ - целая аналитическая функция и справедливы равенства (2.12). Поэтому полагаем

$$
S^{-1}\left(x_{0}^{*}(t), x_{1}^{*}(t)\right)=\left\{\left(x_{n}^{*}\right),\left(v_{n}^{*}\right)\right\} \in \Pi_{\infty}^{*},
$$

где при $n \in \mathbb{Z}$ числа $x_{n}^{*}, v_{n}^{*}$ определены с помошью равенств (2.13). Теорема 2.3 доказана.

Следуя определению 1.5 ( 22, гл. 1$)$, при любом целом $k \geqslant 0$ для произвольного элемента $x^{*}=\left(x_{0}^{*}(t), x_{1}^{*}(t)\right)$, являюшегося парой, состояшей из двух бесконечно-дифференцируемых функций, введем норму

$$
\left\|x^{*}\right\|^{(k)}=\sum_{\nu=0}^{1} \sum_{s=0}^{k} \sup _{t \in \mathbb{R}}\left|\frac{d^{s} x_{\nu}^{*}}{d t^{s}}(t)\right|,
$$

где по определению $\frac{d^{0} x_{\nu}^{*}}{d t^{0}}(t)=x_{\nu}^{*}(t)$, и, следуя определению $1.6(\S 2$, гл. 1$)$, для любых двух элементов $x^{\prime *}=\left\{\left(x_{n}^{\prime}\right),\left(v_{n}^{\prime}\right)\right\} \in \Pi_{\infty}^{*}, x^{\prime \prime *}=\left\{\left(x_{n}^{\prime \prime}\right),\left(v_{n}^{\prime \prime}\right)\right\} \in \Pi_{\infty}^{*}$ введем расстояние

$$
\rho^{(k)}\left(x^{\prime *}, x^{\prime \prime *}\right)=\left\|S x^{\prime *}-S x^{\prime \prime *}\right\|^{(k)},
$$

где $k=0,1,2, \ldots$, а $S$ - отображение, определенное в (2.14). Повторяя определение $1.7(\S 2$, гл. 1$)$, введем множества $\Pi^{(\kappa)}\left(x^{0}\right)$ и $\Pi_{\infty}^{*(\kappa)}\left(x^{0}\right) \subset \Pi_{\infty}^{*}$, где $x^{0}=\left(x_{n}^{0}(t)\right)-$ нулевое решение, введенное в определении 2.2 этого параграфа.

ОПРЕДЕЛЕнИЕ 2.6. Пусть $\kappa=\left(\kappa_{0}, \kappa_{1}, \ldots\right)$ - односторонняя последовательность, состояшая из положительных чисел. Полагаем

$$
\begin{aligned}
\Pi^{(\kappa)}\left(x^{0}\right) & =\left\{x^{*}=\left(x_{0}^{*}(t), x_{1}^{*}(t)\right):\left\|x^{*}-\widetilde{x}^{0}\right\|^{(k)} \leqslant \kappa_{k} ; k=0,1,2 \ldots\right\}, \\
\Pi_{\infty}^{*(\kappa)}\left(x^{0}\right) & =\left\{\widehat{x}=\left\{\left(\widehat{x}_{n}\right),\left(\widehat{v}_{n}\right)\right\} \in \Pi_{\infty}^{*}: \rho^{(k)}\left(\widehat{x}, \widehat{x}^{0}\right) \leqslant \kappa_{k} ; k=0,1,2 \ldots\right\},
\end{aligned}
$$

где $\widetilde{x}^{0}=\left(x_{0}^{0}(t), x_{1}^{0}(t)\right)$ - такой элемент, что $x_{0}^{0}(t) \equiv x_{1}^{0}(t) \equiv 0$, а $\widehat{x}^{0}=\left\{\left(\widehat{x}_{n}^{0}\right),\left(\widehat{v}_{n}^{0}\right)\right\} \in$ $\Pi_{\infty}^{*}$ - такой элемент, что $\widehat{x}_{n}^{0}=\widehat{v}_{n}^{0}=0$ при всех $n \in \mathbb{Z}$. 


\section{§3. Устойчивость нулевого решения и бесконечно-мерный аналог теории КАМ}

В силу определений 2.2-2.5 (§ 2) нулевое решение $x^{0}=\left(x_{n}^{0}(t)\right)$ в пространствах П и $\Pi_{\infty}$ определяется начальными данными

$$
\left(x_{0}^{0}(t), x_{1}^{0}(t)\right)=(0,0) \in \Pi, \quad(0,0) \in \Pi_{\infty}, \quad x_{0}^{0}(t) \equiv 0, \quad x_{1}^{0}(t) \equiv 0,
$$

а в пространстве $\Pi_{\infty}^{*}$ определяется начальными данными

$$
\left\{\left(\widehat{x}_{n}^{0}\right),\left(\widehat{v}_{n}^{0}\right)\right\}=\{(0),(0)\} \in \Pi_{\infty}^{*}, \quad \widehat{x}_{n}^{0}=0, \quad \widehat{v}_{n}^{0}=0, \quad n \in \mathbb{Z} .
$$

Пусть $\kappa=\left(\kappa_{0}, \kappa_{1}, \ldots\right)$ - односторонняя последовательность, состоящая из заранее заданных положительных чисел $\kappa_{s}(s=0,1, \ldots)$. Рассмотрим возмущения начальных данных (2.17), (2.18) следуюшего вида:

$$
\begin{gathered}
\left(x_{0}^{*}(t), x_{1}^{*}(t)\right)=\left(\Delta_{0}(t), \Delta_{1}(t)\right) \in \Pi^{(\kappa)}\left(x^{0}\right), \\
\left\{\left(x_{n}^{*}\right),\left(v_{n}^{*}\right)\right\} \in \Pi_{\infty}^{*(\kappa)}\left(x_{0}\right),
\end{gathered}
$$

где $\Pi^{(\kappa)}\left(x^{0}\right), \Pi_{\infty}^{*(\kappa)}\left(x^{0}\right)$ - множества, введенные в определении $2.6(\S 2) ; \Delta_{0}(t)$ и $\Delta_{1}(t)$ - бесконечно-дифференцируемые функции на прямой $t \in \mathbb{R}$, а $x_{n}^{*}, v_{n}^{*}$ - числа. В силу определения 2.6 соотношение (2.19) эквивалентно неравенствам

$$
\left\|\left(\Delta_{0}(t), \Delta_{1}(t)\right)\right\|^{(k)} \leqslant \kappa_{k} ; \quad k=0,1,2, \ldots,
$$

а соотношение (2.20) эквивалентно неравенствам

$$
\rho^{(k)}\left(\left\{\left(x_{n}^{*}\right),\left(v_{n}^{*}\right)\right\},\{(0),(0)\}\right) \leqslant \kappa_{k} ; \quad k=0,1, \ldots,
$$

где $\|\cdot\|^{(k)}$ - норма, введенная в $(2.15)$, а $\rho^{(k)}(\cdot, \cdot)$ - расстояние в пространстве $\Pi_{\infty}^{*}$, введенное в (2.16).

ТЕОРема 2.4. Предположим, что $m_{n} \leqslant M$ при всех $n \in \mathbb{Z}$, а параметр с в уравнении (2.1) удовлетворяет следующим неравенствам

$$
0<c<4, \quad c \neq 2-2 \cos \frac{2 \pi s}{d}, \quad(s=0, \pm 1, \ldots, d ; \quad d=1 \ldots, r+1) .
$$

Тогда существуют такие константы $m_{n}^{0}>0(n \in \mathbb{Z})$, зависящие только от последовательности $\kappa$ и числа $M$, что если при каком-либо $n_{0} u|n| \geqslant n_{0}$ числа $m_{n}$ удовлетворяют условиям $m_{n} \leqslant m_{n}^{0}$, то нулевое решение $x^{0}$ устойчиво в следующим смысле: для любого $\varepsilon>0$ найдутся $\delta>0$ и натуральное число $k_{0}$, что если выполнено какое-либо из двух условий

а) соотношение $(2.19) u\left\|\left(\Delta_{0}(t), \Delta_{1}(t)\right)\right\|^{\left(k_{0}\right)} \leqslant \delta$,

б) соотношение $(2.20)$ и $\rho^{\left(k_{0}\right)}\left(\left\{\left(x_{n}^{*}\right),\left(v_{n}^{*}\right)\right\},\{(0),(0)\}\right) \leqslant \delta$, то решение $\left(x_{n}(t)\right)$ уравнения (2.1) при всех $n \in \mathbb{Z} u t \in \mathbb{R}$ удовлетворяет неравенству $\left|x_{n}(t)\right| \leqslant \varepsilon$.

Теорема 2.4 будет доказана в следующем параграфе, а здесь мы выведем из нее следствия $2.2,2.3$, теорему 2.5 и ее следствие 2.4 . 
СлЕДСТВИЕ 2.2. Если $m_{n} \leqslant M$ nри всех $n \in \mathbb{Z}$, а параметр с удовлетворяет неравенствам (2.23) теоремы 2.4, то существуют такие константы $m_{n}^{0}>0$ $(n \in \mathbb{Z})$, что если при каком-либо $n_{0}$ u $|n| \geqslant n_{0}$ числа $m_{n}$ удовлетворяют условиям $m_{n} \leqslant m_{n}^{0}$, то пространства $\Pi, \Pi_{\infty} u \Pi_{\infty}^{*}$, введенные в определениях 2.3-2.5, - не пустьл.

ДокАЗАТЕльство. Действительно, в силу определения множества $\Pi^{(\kappa)}$ в определении $2.6(\S 2)$ возмушение $\left(\Delta_{0}(t), \Delta_{1}(t)\right)$ может быть задано с помощью произвольных бесконечно-дифференцируемых или целых аналитических функщий, удовлетворяющих условию (2.19), и согласно утверждению теоремы 2.4 , если $0<\varepsilon<\alpha$, то решение $\left(x_{n}(t)\right)$ уравнения (2.1) при всех $n \in \mathbb{Z}$ и $t \in \mathbb{R}$ будет удовлетворять неравенству (2.8), где $\alpha$ - константа, введенная в (2.9). Теперь следствие 2.2 непосредственно вытекает из определений 2.3-2.5 и теоремы 2.3 (§2). Следствие 2.2 доказано.

СлЕДСТВИЕ 2.3. Предположим, что $0<\varepsilon<1$ и при всех $n \in \mathbb{Z}$ величины $m_{n}$ в уравнении (2.1) удовлетворяют неравенствам $m_{n} \leqslant M$. Тогда существует такая константа $K$, зависящая только от числа $M$ и параметров $c, \beta, r$, входящих в уравнение (2.1), что решения $\left(x_{n}(t)\right)$ уравнения $(2.1)$, найденные в теореме 2.4 и удовлетворяюшие неравенствам $\left|x_{n}(t)\right| \leqslant \varepsilon(n \in \mathbb{Z})$, при всех $n \in \mathbb{Z} u$ $t \in \mathbb{R}$ также удовлетворяют неравенствам $\left|p_{n}(t)\right| \leqslant K \varepsilon$, де $p_{n}(t)=m_{n} \frac{d x_{n}}{d t}(t)$.

ДокАЗАТЕльСтво. Из вида уравнения (2.1) следует, что

$$
\left|\frac{d p_{n}}{d t}(t)\right|<\varepsilon\left(4+c+\beta \cdot 2^{r+1}\right) .
$$

Поэтому утверждение следствия 2.3 вытекает из формулы Тейлора для функции $m_{n} x_{n}(t): m_{n} x_{n}(t+1)=m_{n} x_{n}(t)+p_{n}(t)+\frac{1}{2} \frac{d p_{n}}{d t}(\theta)$, где $t \leqslant \theta \leqslant t+1$. Следствие 2.3 доказано

Теорема 2.4 и следствие 2.3 показывают, что, при малом отклонении начальных данных уравнения (2.1) по норме в соответствуюшем фазовом пространстве от начальных данных нулевого решения, отклонения координат $x_{n}(t)$ и импульсов $p_{n}(t)$ соответствующей гамильтоновой системы от нуля при всех $n \in \mathbb{Z}$ и $t \in \mathbb{R}$ также будут малы.

ТеОРема 2.5 (бесконечно-мерный аналог теории КАМ). Предположим, что величинь $m_{n}$ и параметр с удовлетворяют условию теоремы 2.4. Тогда существуют такие константы $m_{n}^{0}>0(n \in \mathbb{Z})$, что если при каком-либо $n_{0} u|n| \geqslant n_{0}$ числа $m_{n}$ удовлетворяют неравенствам $m_{n} \leqslant m_{n}^{0}$, то при любом $\varepsilon>0$ существуют такие бесконечные множества квази-периодических решений $\left(x_{n}(t)\right)$ уравнения (2.1) с континуальным, счетным и любым конечным количеством рационально независимых частот, что при всех $n \in \mathbb{Z} u t \in \mathbb{R}$ выполняется неравенство $\left|x_{n}(t)\right| \leqslant \varepsilon$.

ДоКАЗАТЕЛЬСТВо. Определим функции $x_{0}^{*}(t), x_{1}^{*}(t)$ в $(2.19)$, используя функции $\Delta_{0}(t), \Delta_{1}(t)$, которые введены в следствии $1.3(\S 5$, гл. 1) с помощью равенств (1.39) и возьмем в качестве чисел $m_{n}^{0}(n \in \mathbb{Z})$ константы, найденные в теореме 2.4. В этом 
случае, если константа $\gamma$, указанная в следствии 1.3 , достаточно мала, то выполняется условие а) теоремы 2.4 , и поэтому если при каком-либо $n_{0}$ и $|n| \geqslant n_{0}$ числа $m_{n}$ удовлетворяют неравенствам $m_{n} \leqslant m_{n}^{0}$, то при любом $\varepsilon>0$ решение $\left(x_{n}(t)\right)$ уравнения (2.1) с начальньми данными $\left(x_{0}^{*}(t), x_{1}^{*}(t)\right)$ при всех $t \in \mathbb{R}, n \in \mathbb{Z}$ удовлетворяет соотношениям

$$
x_{0}(t)=x_{0}^{*}(t), \quad x_{1}(t)=x_{1}^{*}(t), \quad\left|x_{n}(t)\right| \leqslant \varepsilon .
$$

Таким образом, для доказательства теоремы 2.5 осталось показать, что функции $x_{n}(t)$ являются квази-периодическими с указанными в теореме 2.5 количествами рационально-независимых частот. В силу $(2.24),(2.19)$ и равенств $(1.39)$ ( 5 , гл. 1) это утверждение справедливо для функций $x_{0}(t), x_{1}(t)$; предполагая, что оно справедливо для функций $x_{n-1}(t)$ и $x_{n}(t)$, докажем его для функций $x_{n+1}(t)$ и $x_{n-2}(t)$. Предполагая, что число $\varepsilon$ меньше, чем константа $\alpha$, введенная в равенстве $(2.9)$, получим, что в силу (2.24) и (2.8) будет вьполнено утверждение леммы 2.1 (§2), и поэтому равенства (2.10) и (2.11) при фиксированном значении $t$ определяют величину $z_{n}(t)=z_{n}\left(\xi_{n}(t)\right)$ как аналитическую функцию от $\xi_{n}(t)$ и величины $x_{n+1}(t), x_{n-2}(t)$ как аналитические функщии от $x_{n}(t)$ и $x_{n-1}(t)$. Из этого утверждения в силу определений 7)-11) (см. общие обозначения и определения) и следует, что при всех $n \in \mathbb{Z}$ функции $x_{n}(t)$ будут квази-периодическими с тем же набором частот, что и функции $\Delta_{0}(t)$ и $\Delta_{1}(t)$. Теорема 2.5 доказана.

СлЕДСТВИЕ 2.4. Если величины $m_{n}$ и параметр с удовлетворяют условию теоремы 2.4 и при $|n| \geqslant n_{0}$ справедливы неравенства $m_{n} \leqslant m_{n}^{0}\left(m_{n}^{0}-\right.$ константы, найденные в теореме 2.4), то для любого $\varepsilon>0$ в области $\Gamma_{\varepsilon}^{\prime}=$ $\left\{\vec{x}=\left(x_{n}\right):\left|x_{n}\right| \leqslant \varepsilon, n \in \mathbb{Z}\right\}$ существуют инвариантные множсества, составленные из решений уравнения (2.1), которые представляют собой торы любой конечной размерности, счетно-мерные торь и существенное подмножество тора континуальной размерности, а решения уравнения (2.1), расположсенные на этих торах, порождают на них квази-периодические движения.

Следствие 2.4 вьводится из теоремы 2.5 точно так же, как следствие 1.5 вьводилось из теоремы 1.11 ( 5 , гл. 1$)$.

\section{§4. Доказательство теоремы 2.4}

Доказательство проводится по тому же пути, что и доказательство теоремы 1.10 $(\S 6$, гл. 1), однако есть существенные отличия, связанные с формой нелинейных членов в уравнении (2.1).

Лемма 2.2. Существуют константы $m_{n}^{0}>0(n \in \mathbb{Z})$ и число $\varepsilon_{0}>0$ такие, что если $m_{n} \leqslant M$ при всех $n \in \mathbb{Z}$, а при $|n| \geqslant n_{0} m_{n} \leqslant m_{n}^{0}$, то в случае выполнения соотношения (2.19) для любого решения $\left(x_{n}(t)\right)$ уравнения (2.1), удовлетворяющего при некоторых иельх числах $N_{1} \leqslant 0, N_{2} \geqslant 1$ соотношениям

$$
\sup _{t \in \mathbb{R}}\left|x_{n}(t)\right| \leqslant \varepsilon_{0}, \quad N_{1} \leqslant n \leqslant N_{2},
$$


будет справедливо неравенство

$$
\sum_{n=N_{1}-1}^{N_{2}+1} m_{n} \sup _{t \in \mathbb{R}}\left|\frac{d^{2} x_{n}}{d t^{2}}(t)\right|<c^{*},
$$

где $c^{*}$ - константа, не зависящая от чисел $N_{1}$ и $N_{2}$ и от начальных данных.

ДокАЗАТЕЛЬСТВо. В силу равенств (2.10) и (2.11) и определения 2.6 (§ 2$)$ из неравенств (2.25) при всех $t \in \mathbb{R}$ следуют неравенства

$$
\left|\frac{d^{2} x_{N_{2}+1}}{d t^{2}}(t)\right|<c_{N}, \quad\left|\frac{d^{2} x_{N_{1}-1}}{d t^{2}}(t)\right|<c_{N},
$$

где $N=\max \left(\left|N_{1}\right|+3, N_{2}+2\right), c_{N}$ - константа, зависящая только от числа $M$ и от первых $N+1$ чисел $\kappa_{0}, \ldots, \kappa_{N}$, входящих в последовательность $\kappa$, для которой справедливо соотношение (2.19). Так как число $M$ и последовательность $\kappa$ заранее зафиксированы, то из неравенства (2.27) очевидно следует утверждение леммы 2.2. Лемма 2.2 доказана.

Предположим, что $\left(x_{n}(t)\right)$ есть решение уравнения (2.1). Введем преобразование $A$ и две последовательности семейств преобразований $B_{k}^{\prime}(t), B_{k}^{\prime \prime}(t)(k=1,2, \ldots)$ плоскости $\xi, \theta$, зависяших от параметра $t$. Обозначая через $z=h(\xi)$ функцию, обратную к функции

$$
\xi=z+\beta z^{r}
$$

в достаточно малой окрестности точки $z=0$, полагаем

$$
A:(\xi, \theta) \rightarrow\left(\xi^{\prime}, \theta^{\prime}\right)=\left(\xi-c \theta, \theta+h\left(\xi^{\prime}\right)\right)
$$

$$
\begin{aligned}
& B_{k}^{\prime}(t):(\xi, \theta) \rightarrow\left(\xi_{k}^{\prime}(t), \theta_{k}^{\prime}(t)\right)=\left(\xi+m_{k} \frac{d^{2} x_{k}}{d t^{2}}(t), \theta+h\left(\xi_{k}^{\prime}(t)\right)-h(\xi)\right) \\
& B_{k}^{\prime \prime}(t):(\xi, \theta) \rightarrow\left(\xi_{k}^{\prime \prime}(t), \theta_{k}^{\prime \prime}(t)\right)=\left(\xi-m_{-k+1} \frac{d^{2} x_{k}}{d t^{2}}(t), \theta+h\left(\xi_{k}^{\prime \prime}(t)\right)-h(\xi)\right) .
\end{aligned}
$$

Вводя функции

$$
\begin{array}{ll}
\xi_{n+1}^{\prime}(t)=q_{n+1}(t)-q_{n}(t)+\beta\left(q_{n+1}(t)-q_{n}(t)\right)^{r} & (n=0,1,2 \ldots), \\
\xi_{n-1}^{\prime \prime}(t)=q_{n-1}(t)-q_{n}(t)+\beta\left(q_{n-1}(t)-q_{n}(t)\right)^{r} & (n=1,0,-1,-2 \ldots),
\end{array}
$$

мы, используя уравнение (2.1) и нечетность числа $r$, получим следуюшие равенства

$$
\begin{array}{ll}
\left(\xi_{n+1}^{\prime}(t), x_{n+1}(t)\right)=B_{n}^{\prime}(t) \circ A\left(\xi_{n}^{\prime}(t), x_{n}(t)\right) & (n=1,2, \ldots), \\
\left(\xi_{n-1}^{\prime \prime}(t), x_{n-1}(t)\right)=B_{1-n}^{\prime \prime}(t) \circ A\left(\xi_{n}^{\prime \prime}(t), x_{n}(t)\right) & (n=0,-1,-2, \ldots),
\end{array}
$$

которые приводят к равенствам

$$
\begin{gathered}
\left(\xi_{k}^{\prime}(t), x_{k}(t)\right)=B_{k-1}^{\prime}(t) \circ A \circ B_{k-2}^{\prime}(t) \circ A \circ \cdots \circ B_{1}^{\prime}(t) \circ A\left(\xi_{1}^{\prime}(t), x_{1}(t)\right) \\
(k=2,3 \ldots), \\
\left(\xi_{s}^{\prime \prime}(t), x_{s}(t)\right)=B_{-s}^{\prime \prime}(t) \circ A \circ B_{-s-1}^{\prime \prime}(t) \circ A \circ \cdots \circ B_{1}^{\prime \prime}(t) \circ A\left(\xi_{0}^{\prime \prime}(t), x_{0}(t)\right) \\
(s=-1,-2, \ldots) .
\end{gathered}
$$


Лемма 2.3. Предположим, что нечетное число $r \geqslant 3$ в уравнении (2.1) имеет вид $r=2 l+1$. Тогда при выполнении условий (2.23) теоремы 2.4 (§ 3) преобразование $A$, введенное в равенстве (2.29), сохраняет площадь, а точка $(0,0)$ является его неподвижной точкой, в которой $A$ имеет общий эллиптический тип, так что в полярных координатах $\psi, \tau$ в нормальной форме Биркгофа [52, $\S 21]$

$$
\psi^{\prime}=\psi+\omega_{0}+\omega_{1} \tau+\cdots+\omega_{l} \tau^{l}+\cdots, \quad \tau^{\prime}=\tau
$$

$(\psi-$ угол, $\tau-$ радиус $)$ коэффичиенть

$$
\omega_{k}=0 \quad(k=1, \ldots, l-1),
$$

а коэффициент

$$
\omega_{l} \neq 0
$$

ДокАЗАТЕльСТво. Так как функция $z=h(\xi)$ является обратной к функции $(2.28)$, то $h(0)=0$, и поэтому в силу $(2.29)$ точка $(0,0)$ является неподвижной для преобразования $A$. Далее, согласно $(2.28)$ и $(2.29)$, линейная часть $d A(0,0)$ отображения $A$ в точке $(0,0)$ имеет вид

$$
d A(0,0)=\left(\begin{array}{cc}
1 & -c \\
1 & 1-c
\end{array}\right)
$$

и, следовательно, $A$ сохраняет площадь, а из первого неравенства в $(2.23)$ следует, что $|\operatorname{Sp} A|<2(\operatorname{Sp} A-$ след $A)$, собственные числа $\lambda$ и $\bar{\lambda}$ - различные, комплексно-сопряженные числа и $|\lambda|=1$. Таким образом, неподвижная относительно $A$ точка $(0,0)$ имеет эллиптический тип. Докажем теперь, что точка $(0,0)$ является точкой обшего эллиптического типа, и справедливость $(2.34)-(2.36)$. Приводя с помощью матришы

$$
B=\left(\begin{array}{cc}
1 & 1 \\
-\frac{\lambda-1}{c} & -\frac{\bar{\lambda}-1}{c}
\end{array}\right) \text { и замены }\left(\begin{array}{l}
\xi \\
\theta
\end{array}\right)=B\left(\begin{array}{l}
q \\
p
\end{array}\right)
$$

матрицу $d A(0,0)$ в $(2.37)$ к диагональному виду, получим, что преобразование

$$
D=B^{-1} \circ A \circ B:(q, p) \rightarrow\left(q^{\prime}, p^{\prime}\right)
$$

имеет следуюший вид

$$
\left\{\begin{array}{l}
q^{\prime}=\lambda q+\frac{c}{\bar{\lambda}-\lambda} \sum_{k=2}^{\infty} \frac{(\lambda q+\bar{\lambda} p)^{k}}{k !} \frac{d^{k} h}{d \xi^{k}}(0) \\
p^{\prime}=\bar{\lambda} q-\frac{c}{\bar{\lambda}-\lambda} \sum_{k=2}^{\infty} \frac{(\lambda q+\bar{\lambda} p)^{k}}{k !} \frac{d^{k} h}{d \xi^{k}}(0) .
\end{array}\right.
$$

Известно (см. [51, 21$])$, что если

$$
\lambda \neq e^{2 \pi i \frac{s}{d}} \quad(s=0, \pm 1, \ldots, \pm d ; d=1, \ldots, 2 l+2),
$$


где $i$ - мнимая единица, то сушествует замена переменных

$$
Q:(\xi, \eta) \rightarrow(q, p)
$$

имеющая вид

$$
\left\{\begin{array}{l}
q=f(\xi, \eta)=\xi+\sum_{k=2}^{\infty} f_{k} \\
p=g(\xi, \eta)=\eta+\sum_{k=2}^{\infty} g_{k}
\end{array}\right.
$$

$\left(f_{k}\right.$ и $g_{k}$ однородные многочлены степени $k$ по совокупности переменных $\left.\xi, \eta\right)$, которая приводит преобразование $D$, определенное равенствами $(2.38),(2.39)$, к виду

$$
L=Q^{-1} \circ D \circ Q:(\xi, \eta) \rightarrow\left(\xi^{\prime} \eta^{\prime}\right)
$$

где $Q^{-1}$ - отображение, обратное к $Q$,

$$
\begin{aligned}
\xi^{\prime} & =u \xi+\widetilde{u}(\xi, \eta), & \eta^{\prime} & =v \eta+\widetilde{v}(\xi, \eta), \\
u & =\lambda+\sum_{k=1}^{l} \alpha_{k}(\xi \eta)^{k}, & v & =\bar{\lambda}+\sum_{k=1}^{l} \beta_{k}(\xi \eta)^{k}
\end{aligned}
$$

$u v=1 ; \alpha_{k}, \beta_{k}(k=1, \ldots, l)-$ коэффициенты, не зависяшие от $\xi, \eta ; \widetilde{u}(\xi, \eta), \widetilde{v}(\xi, \eta)-$ сходящиеся ряды, начинаюшиеся с членов порядка $2 l+2$ по совокупности переменных $\xi, \eta$. В нашем случае из второго неравенства в $(2.23)$, из вида матрицы $d A(0,0)$ в $(2.37)$ и равенства $r=2 l+1$ следует, что собственное число $\lambda$ матрицы $d A(0,0)$ удовлетворяет неравенствам (2.40), и поэтому замена переменных $(2.41)$, для которой выполнены соотношения $(2.42)-(2.45),-$ существует. Производя сравнение коэффициентов в равенствах $(2.43),(2.44)$ при использовании $(2.38),(2.39),(2.41),(2.42)$ и $(2.45)$ с помощью метода из $[51, \S 21]$ и учитывая, что в силу $(2.28)$ в равенствах $(2.39)$ величина $\frac{d^{k} h}{d \xi^{k}}(0)=0$, если $2 \leqslant k \leqslant r-1$, a $\frac{d^{r} h}{d \xi^{r}}(0) \neq 0$, получим, что в равенствах $(2.45)$

$$
\begin{aligned}
& \alpha_{k}=\beta_{k}=0 \quad(1 \leqslant k \leqslant l-1), \\
& \alpha_{l}=\frac{c \lambda}{(\bar{\lambda}-\lambda) l !}\left(\begin{array}{l}
r \\
l
\end{array}\right) \frac{d^{r} h}{d \xi^{r}}(0) \neq 0,
\end{aligned}
$$

где $\left(\begin{array}{l}r \\ l\end{array}\right)$ - биномиальньй коэффициент. Вводя теперь полярные координаты $\psi, \tau$ посредством равенств $\xi=\sqrt{2 \tau} e^{i \psi}, \eta=\sqrt{2 \tau} e^{-i \psi}$ и выражая через них преобразование $L$, введенное в $(2.43)$, в силу (2.44)-(2.47) получим соотношения (2.34)-(2.36). Лемма 2.3 доказана. 
Представим преобразования $B_{k}^{\prime}(t)$ и $B_{k}^{\prime \prime}(t)$, определенные равенствами (2.30) и $(2.31)$, в виде

$$
\left\{\begin{array}{l}
B_{k}^{\prime}(t):(\xi, \theta) \rightarrow\left(\xi_{k}^{\prime}(t), \theta_{k}^{\prime}(t)\right)=\left(\xi+b_{1 k}^{\prime}(\xi, \theta, t), \theta+b_{2 k}^{\prime}(\xi, \theta, t)\right), \\
B_{k}^{\prime \prime}(t):(\xi, \theta) \rightarrow\left(\xi_{k}^{\prime \prime}(t), \theta_{k}^{\prime \prime}(t)\right)=\left(\xi+b_{1 k}^{\prime \prime}(\xi, \theta, t), \theta+b_{2 k}^{\prime \prime}(\xi, \theta, t)\right),
\end{array}\right.
$$

возьмем в качестве величин $\varepsilon_{0}$ и $m_{n}^{0}(n \in \mathbb{Z})$ константы, указанные в лемме 2.2 , и будем применять к двум множествам преобразований $A, B_{k}^{\prime}(t)(k=1,2, \ldots)$ и $A, B_{k}^{\prime \prime}(t)(k=1,2, \ldots)$ теорему 2.6 , сформулированную в следующем $\S 5$. Если бы в области $V_{\varepsilon_{0}}=\left\{\xi, \theta: \max (|\xi|,|\theta|) \leqslant \varepsilon_{0}\right\}$ было справедливо неравенство

$$
\sum_{k=1}^{\infty} \sup _{\substack{(\xi, \theta) \in V_{\varepsilon_{0}} \\ t \in \mathbb{R}}}\left(\left|b_{1 k}^{\prime}(\xi, \theta, t)\right|+\left|b_{2 k}^{\prime}(\xi, \theta, t)\right|+\left|b_{1 k}^{\prime \prime}(\xi, \theta, t)\right|+\left|b_{2 k}^{\prime \prime}(\xi, \theta, t)\right|\right)<\infty
$$

то в силу леммы 2.3 каждое из двух множеств преобразований $A, B_{k}^{\prime}(t)(k=1,2, \ldots)$ и $A, B_{k}^{\prime \prime}(t)(k=1,2, \ldots)$ удовлетворяло бы условию теоремы 2.6 (§ 5$)$ и, применяя эту теорему, мы бы получили следующее утверждение: для любого $\varepsilon>0$ найдется $\widehat{\delta}>0$, удовлетворяющее условию $\widehat{\delta}<\varepsilon$, и натуральные числа $n^{\prime}, n^{\prime \prime}$ такие, что если $|\xi| \leqslant \widehat{\delta}$, $|\theta| \leqslant \widehat{\delta}$, то для любых натуральных чисел $k^{\prime}, k^{\prime \prime}$ с условиями $k^{\prime} \geqslant n^{\prime}$ и $k^{\prime \prime} \geqslant n^{\prime \prime}$ и для любого $t \in \mathbb{R}$

$$
\left\{\begin{array}{l}
\left|B_{k^{\prime}}^{\prime}(t) \circ A \circ \cdots \circ B_{n^{\prime}}^{\prime}(t) \circ A(\xi, \theta)\right| \leqslant \varepsilon, \\
\left|B_{k^{\prime \prime}}^{\prime \prime}(t) \circ A \circ \cdots \circ B_{n^{\prime \prime}}^{\prime \prime}(t) \circ A(\xi, \theta)\right| \leqslant \varepsilon .
\end{array}\right.
$$

Из этого же последнего утверждения и равенств (2.32), (2.33) следует заключение теоремы 2.4 , так как в силу $(2.1)$, леммы 2.1 (§ 2$)$ и равенств $(2.10),(2.11),(2.15),(2.16)$ для любого $\widehat{\delta}>0$ найдутся такое $\delta>0$ и натуральное число $k_{0}$, что если выполнено какое-либо из двух неравенств

$$
\left\|\left(\Delta_{0}(t), \Delta_{1}(t)\right)\right\|^{\left(k_{0}\right)} \leqslant \delta, \quad \rho^{\left(k_{0}\right)}\left(\left\{\left(x_{n}^{*}\right),\left(v_{n}^{*}\right)\right\},\{(0),(0)\}\right) \leqslant \delta,
$$

то для всех $t \in \mathbb{R}$

$$
\begin{cases}\left|B_{s}^{\prime}(t) \circ A \circ \cdots \circ B_{1}^{\prime}(t) \circ A\left(\xi_{1}^{\prime}(t), x_{1}(t)\right)\right| \leqslant \widehat{\delta} \quad\left(1 \leqslant s \leqslant n^{\prime}-1\right), \\ \left|B_{s}^{\prime \prime}(t) \circ A \circ \cdots \circ B_{1}^{\prime \prime}(t) \circ A\left(\xi_{0}^{\prime}(t), x_{0}(t)\right)\right| \leqslant \widehat{\delta} \quad\left(1 \leqslant s \leqslant n^{\prime \prime}-1\right) .\end{cases}
$$

Таким образом, доказательство теоремы 2.4 сводится к доказательству неравенства $(2.49)$, что в силу $(2.48)$ и определений преобразований $B_{k}^{\prime}(t)$ и $B_{k}^{\prime \prime}(t)$ в $(2.30)$ и (2.31) сводится к доказательству при любых целых числах $N_{1}, N_{2}$ с условиями $N_{1} \leqslant 0, N_{2} \geqslant 1$ неравенства

$$
\sum_{n=N_{1}}^{N_{2}} m_{n} \sup _{t \in \mathbb{R}}\left|\frac{d^{2} x_{n}}{d t^{2}}(t)\right|<c^{*}
$$


для решений с начальньми данными, указанньми в формулировке теоремы 2.4 , где константа $c^{*}$ не зависит от этих начальных данных и от чисел $N_{1}$ и $N_{2}$. Выведем неравенство (2.52) из леммы 2.2 и теоремы 2.6 (§5). Задавая число $\varepsilon$ так, чтобы $0<\varepsilon<\varepsilon_{0}$ $\left(\varepsilon_{0}\right.$ введено в условии леммы 2.2$)$, и исходя из предположения о справедливости неравенства (2.52) с константой $c^{*}$, найденной в лемме 2.2 , выберем по этому $\varepsilon$ натуральные числа $n^{\prime}, n^{\prime \prime}, k_{0}$ и числа $\widehat{\delta}>0, \delta>0$ так, чтобы $\widehat{\delta}<\varepsilon$ и чтобы выполнялись неравенства $(2.50)$ и (2.51). При этом отметим, что при заданных числах $n^{\prime}, n^{\prime \prime}$ и $\widehat{\delta}$ определение чисел $k_{0}$ и $\delta$, для которых справедливы неравенства а), б) в условии теоремы 2.4 и неравенства (2.51), не связано с неравенством (2.52) и всегда может быть осушествлено. Поэтому в силу $(2.51),(2.32),(2.33)$ для всех целых чисел $n$, удовлетворяюших условию $1-n^{\prime \prime} \leqslant n \leqslant n^{\prime}$, имеем неравенство

$$
\sup _{t \in \mathbb{R}}\left|x_{n}(t)\right| \leqslant \varepsilon_{0}
$$

и, согласно лемме 2.2, неравенство (2.52) выполняется для чисел $N_{1}=-n^{\prime \prime}$, $N_{2}=n^{\prime}+1$. Далее, предполагая, что неравенство (2.52) справедливо для некоторых чисел $N_{1} \leqslant-n^{\prime \prime}, N_{2} \geqslant n^{\prime}+1$, в силу леммы 2.2 и теоремы $2.6(\S 5)$ получим, что неравенства (2.50) вьполняются для всех целых чисел $k^{\prime}, k^{\prime \prime}$, удовлетворяюших условиям $n^{\prime} \leqslant k^{\prime} \leqslant N_{2}-1, n^{\prime \prime} \leqslant k^{\prime \prime} \leqslant-N_{1}$, и, следовательно, в силу (2.32) и (2.33) неравенство (2.53) справедливо для чисел $n$, удовлетворяющих условию $N_{1} \leqslant n \leqslant N_{2}$. Применяя теперь еще раз лемму 2.2 , получим, что неравенство $(2.52)$ справедливо для чисел $N_{1}-1, N_{2}+1$ вместо чисел $N_{1}, N_{2}$ соответственно, а значит, и для любых целых чисел $N_{1}, N_{2}$, удовлетворяюших неравенствам $N_{1} \leqslant 0, N_{2} \geqslant 1$. Теорема 2.4 доказана.

\section{§ 5. Об устойчивости относительно неавтономной динамической системы с дискретным временем}

Здесь будет сформулирована теорема 2.6, касаюшаяся поведения неавтономных динамических систем с дискретным временем, которая использовалась при доказательствах теорем 1.10 ( 6 , гл. 1), 2.4 ( $(4$, гл. 2) и применяется также в доказательствах теорем 3.4 и 3.5 (§2, гл. 3$)$.

ТЕорема 2.6. Пусть $A$-взаимно-однозначное, аналитическое и сохраняющее площадь преобразование окрестности $V_{\varepsilon_{0}}=\left\{q, p: \max (|q|,|p|) \leqslant \varepsilon_{0}\right\} \quad\left(\varepsilon_{0}>0\right)$ плоскости $q, p$, оставляющее на месте точку $O=(0,0), \quad a \quad B_{n}=B_{n}(t)$ $(n=1,2, \ldots)$ - последовательность гомеоморфизмов области $V_{\varepsilon_{0}}$, зависяших от параметра $t \in \mathbb{R}$ и имеющих вид

$$
B_{n}:(q, p) \rightarrow\left(q+b_{1}^{(n)}(q, p, t), p+b_{2}^{(n)}(q, p, t)\right)
$$

Предположим, что выполнень следующие условия:

1) А - отображсение общего эллиптического типа, т.е. в его нормальной форме Биркгофа $[51, \S 21]$ 


$$
\psi^{\prime}=\psi+\omega_{0}+\omega_{1} \tau+\cdots+\omega_{l} \tau^{l}+\cdots, \quad \tau^{\prime}=\tau
$$

$(\psi, \tau$ - полярные координаты ; $\psi$ - угол, $\tau$ - радиус $)$ при некотором натуральном числе l коэффициент $\omega_{l} \neq 0$;

2) коэффициент $\omega_{0}$ в (2.55) удовлетворяет неравенствам

$$
\omega_{0} \neq 2 \pi \frac{s}{d}, \quad \text { əде } \quad s=0, \pm 1, \ldots, \pm d ; \quad d=1, \ldots, 2 l+2
$$

3) функции $b_{1}^{(n)}(q, p, t), b_{2}^{(n)}(q, p, t)$, в (2.54) удовлетворяют неравенству

$$
\sum_{n=1}^{\infty} \sup _{\substack{(q, p) \in V_{\varepsilon_{0}} \\ t \in \mathbb{R}}}\left(\left|b_{1}^{(n)}(q, p, t)\right|+\left|b_{2}^{(n)}(q, p, t)\right|\right)<\infty .
$$

Тогда для любого в существуют такие $\delta>0$ и натуральное число $\widehat{n}=\widehat{n}(\varepsilon)$, что если $(q, p) \in V_{\delta}$, то при любом $n \geqslant \widehat{n}$ и $t \in \mathbb{R}$ точка

$$
B_{n} \circ A \circ B_{n-1} \circ A \circ \cdots \circ B_{\widehat{n}} \circ A(q, p) \in V_{\varepsilon} .
$$

ЗАмЕчАниЕ 2.2 . В случае, когда в условии 1 ) теоремы 2.6 число $l=1$, при фиксированном значении $t$ теорема 2.6 является частным случаем теоремы 2 из работы $[44, \S 1$, гл. V, ч. 2]. Доказательство теоремы 2.6 повторяет доказательство более общей теоремы 1 из работы [45, $\S 2]$, соответствующей случаю $l=1$.

\section{§6. Локальная неоднозначная разрешимость и неразрешимость уравнения для стандартного фазового пространства}

В этом параграфе основной результат данной главы об устойчивости нулевого решения, сформулированный в теореме $2.4(\S 3)$ и доказанный в $\S 4$, применяется для исследования проблемы однозначной разрешимости уравнения (2.1) в стандартной постановке. При этом главньй вывод, который проистекает из доказанной ниже теоремы 2.7, состоит в том, что эта проблема имеет отрицательное решение даже в локальной ситуации, т.е. для решений уравнения (2.1), сосредоточенных в сколь угодно малой окрестности нулевого решения.

ОПРЕДЕЛЕниЕ 2.7. Введем пространство П* начальных данных уравнения (2.1), точкой которого $\left\{\left(x_{n}^{*}\right),\left(v_{n}^{*}\right)\right\}$ является пара двусторонних последовательностей $\left(x_{n}^{*}\right)$, $\left(v_{n}^{*}\right)(n \in \mathbb{Z})$ чисел таких, что $x_{n}^{*}=x_{n}\left(t^{*}\right), v_{n}^{*}=\frac{d x_{n}}{d t}\left(t^{*}\right)$, где $t^{*}$ - начальный момент времени. 
Теорема 2.7. Предположим, что $m_{n} \leqslant M$ при всех $n \in \mathbb{Z}$, а параметр с в уравнении (2.1) удовлетворяет условиям (2.23) теоремы 2.4. Тогда существуют такие константы $m_{n}^{0}>0(n \in \mathbb{Z})$, что, если при каком-либо $n_{0} u|n| \geqslant n_{0}$ справедливы неравенства $m_{n} \leqslant m_{n}^{0}$, то для любого $\varepsilon>0$ существуют начальные данные $\left\{\left(x_{n}^{*}\right),\left(v_{n}^{*}\right)\right\} \in \Pi^{*}$, которые определяют бесконечное множество различных решений $\left(x_{n}(t)\right)$ уравнения (2.1) в классе бесконечно-дифферениируемых функиий $x_{n}(t)$ на прямой $t \in \mathbb{R}$, удовлетворяющих при всех $n \in \mathbb{Z}$ условиям

$$
\begin{gathered}
x_{n}\left(t^{*}\right)=x_{n}^{*}, \quad \frac{d x_{n}}{d t}\left(t^{*}\right)=v_{n}^{*}, \\
\sup _{t \in \mathbb{R}}\left|x_{n}(t)\right| \leqslant \varepsilon,
\end{gathered}
$$

а в классе аналитических функиий $x_{n}(t)$ для тех жее начальных данных решение уравнения (2.1) не существует в сколь угодно малой окрестности значения $t^{*}$.

ДокАЗАТЕльство. Возьмем в качестве $m_{n}^{0}$ константы, для которых справедливо утверждение теоремы 2.4 , и рассмотрим одностороннюю последовательность $\kappa=$ $\left(\kappa_{0}, \kappa_{1}, \ldots\right)$, состоящую из положительных чисел, которая обладает следуюшим свойством: если $f(t)$ - такая бесконечно-дифференщируемая функция в окрестности значения $t=t^{*}$, что при некотором $k_{0}>0$

$$
\frac{d^{k} f}{d t^{k}}\left(t^{*}\right)=\kappa_{k}, \quad k>k_{0}
$$

то $f(t)$ не является аналитической функцией $t$ ни в какой окрестности числа $t^{*}$. Далее, для заданного $\varepsilon>0$ найдем константу $\delta>0$ и целое $k_{0}$ такие, что для них выполняется заключение теоремы 2.4. В силу леммы 1.1 (§ 1, гл. 1$)$ и $(2.15)$ сушествует бесконечное множество различных пар бесконечно-дифференцируемых на прямой $t \in \mathbb{R}$ функций $\Delta_{0}(t), \Delta_{1}(t)$, у которых при любом целом $k>k_{0}$ все значения $k$-й производной в точке $t^{*}$ совпадают и равны $\kappa_{k}$, а для целых $k$, удовлетворяюших неравенству $0 \leqslant k \leqslant k_{0}$, значения $k$-й производной в точке $t^{*}$ всех функций, входяших в указанное множество пар, также совпадают, и при этом справедливо условие а) теоремы 2.4 (здесь нулевая производная - это значение функции в точке $t^{*}$ ). Согласно утверждению теоремы 2.4 и (2.19), для каждой такой пары начальных данных $\left(\Delta_{0}(t), \Delta_{1}(t)\right)$ существует решение $\left(x_{n}(t)\right)$ уравнения $(2.1)$, удовлетворяющее неравенству $(2.57)$, а из леммы 2.1 ( $(2)$ следует, что при любом целом $k \geqslant 0$ и фиксированном $n$ значения $k$-й производной в точке $t^{*}$ функций $x_{n}(t)$ во всех парах совпадают. Поэтому справедливы равенства (2.56), в которых при всех $n \in \mathbb{Z}$ числа $x_{n}^{*}, v_{n}^{*}$ не зависят от пары функций, и, таким образом, бесконечное множество различных бесконечно-дифференцируемых решений уравнения (2.1), удовлетворяюших (2.56) и (2.57), построено. Согласно же определению последовательности $\kappa$, не сушествует решения $\left(x_{n}(t)\right)$ уравнения $(2.1)$, удовлетворяюшего (2.56), которое является аналитическим в какой-либо окрестности числа $t^{*}$. Теорема 2.7 доказана. 


\section{Глава 3. \\ Бесконечно-мерные обыкновенные дифференциальные уравнения общего вида}

В этой главе изучаются дифференциальные уравнения, являющиеся возмущениями уравнений (0.1) и (0.2) с помощью функций обшего вида, которые зависят от произвольного числа переменных $q_{n}$ или $x_{n}$. С этой целью введем бесконечно-мерные векторы $\vec{q}=\left(q_{n}\right), \vec{x}=\left(x_{n}\right)$, у которых при $n \in \mathbb{Z} n$-я координата есть, соответственно, $q_{n}$ и $x_{n}$, и рассмотрим следуюшие уравнения

$$
m_{n} \frac{d^{2} q_{n}}{d t^{2}}=\varphi\left(q_{n}\right)+q_{n+1}-2 q_{n}+q_{n-1}+\psi_{n}(\vec{q}),
$$

$$
m_{n} \frac{d^{2} x_{n}}{d t^{2}}=x_{n+1}-(2-c) x_{n}+x_{n-1}+\beta\left[\left(x_{n+1}-x_{n}\right)^{r}-\left(x_{n}-x_{n-1}\right)^{r}\right]+\psi_{n}(\vec{x})
$$

где числа $m_{n}, c, \beta, r$ и целая аналитическая функция $\varphi(q)$ удовлетворяют условиям, указанным во введении, $\psi_{n}(\vec{q})$ - функция от всех переменных $q_{n}$, а $\psi_{n}(\vec{x})$ - результат подстановки в нее в качестве аргумента вместо вектора $\vec{q}$ - вектора $\vec{x}$.

\section{§ 1. Формальная однозначная разрешимость и единственность аналитических решений}

ОПРЕДЕЛЕНИЕ 3.1. Введем пространство $W$ функций $\psi(\vec{q})$ таких, что аргументом функции $\psi(\vec{q})$ является бесконечно-мерный вектор $\vec{q}$, но все ее значения зависят только от конечного числа координат вектора $\vec{q}$, и при этом $\psi(\vec{q})$ есть целая аналитическая функция по совокупности этих координат, т.е. разлагается по ним в абсолютно сходяшийся ряд.

В дальнейшем на протяжении всей этой главы будем предполагать, что при всех $n \in \mathbb{Z}$ функщии $\psi_{n}(\vec{q})$ и $\psi_{n}(\vec{x})$ в уравнениях $(3.1)$ и $(3.2)$ принадлежат пространству $W$.

ЗАмечАниЕ 3.1 . Количество координат векторов $\vec{q}$ и $\vec{x}$, от которых зависят функции $\psi_{n}(\vec{q})$ и $\psi_{n}(\vec{x})$ в уравнениях $(3.1)$ и $(3.2)$, и сами эти координаты могут быть разными при разных значениях переменной $n$.

Далее будут установлены теоремы 3.1 и 3.2 о формальной однозначной разрешимости уравнений (3.1) и (3.2), т.е. об однозначной разрешимости этих уравнений в классе формальных степенных рядов по переменной $t$ в пространствах начальных данных $\Omega^{*}$ и $\Pi^{*}$, введенных в определении 1.1 (§1, гл. 1) и в определении 2.7 (§6, гл. 2).

ТеОРема 3.1. Для любой точки $\left\{\left(q_{n}^{*}\right),\left(v_{n}^{*}\right)\right\} \in \Omega^{*}$ и любого числа $t^{*}$ существуют единственные формальные степеннье ряды

$$
q_{n}(t)=\sum_{k=0}^{\infty} q_{n}^{(k)}\left(t-t^{*}\right)^{k}, \quad n \in \mathbb{Z}
$$


в которых при всех $n \in \mathbb{Z}$ и иельх $k \geqslant 0$ числа $q_{n}^{(k)}$ не зависят от $t, q_{n}^{(0)}=q_{n}^{*}$, $q_{n}^{(1)}=v_{n}^{*}$, и такие, что при подстановке выражсений (3.3) в уравнение (3.1) коэффичиенты при одинаковых степенях $\left(t-t^{*}\right)^{k}$ в обоих частях равенства (3.1) совпадают.

Теорема 3.2. Для любой точки $\left\{\left(x_{n}^{*}\right),\left(v_{n}^{*}\right)\right\} \in \Pi^{*}$ и любого числа $t^{*}$ существуют и единственны формальнье степенные ряды

$$
x_{n}(t)=\sum_{k=0}^{\infty} x_{n}^{(k)}\left(t-t^{*}\right)^{k}, \quad n \in \mathbb{Z}
$$

в которых при всех $n \in \mathbb{Z}$ и чельх $k \geqslant 0$ числа $x_{n}^{(k)}$ не зависят от $t, x_{n}^{(0)}=$ $x_{n}^{*}, x_{n}^{(1)}=v_{n}^{*}$ и такие что при подстановке выражсений (3.4) в уравнение (3.2) коэффичиенты при одинаковых степенях $\left(t-t^{*}\right)^{k}$ в обоих частях равенства (3.2) совпадают.

Доказательства теорем 3.1 и 3.2 основанына том обстоятельстве, что из вида уравнений (3.1) и (3.2) и определения 3.1 следует, что, зная значения всех функций $q_{n}\left(t^{*}\right)$, $x_{n}\left(t^{*}\right)$ и их первых производных в точке $t^{*}$, можно однозначно определить значения всех производных от всех функций $q_{n}(t), x_{n}(t)$ в точке $t^{*}$ и, таким образом, однозначно построить ряды (3.3) и (3.4). То, что ряды (3.3) и (3.4) удовлетворяют уравнениям (3.1) и (3.2), непосредственно следует из их построения.

ЗАмечАниЕ 3.2. Если в уравнениях (3.1) и (3.2) при всех $n \in \mathbb{Z}$ функция $\psi_{n}(\vec{q}) \equiv 0$, а начальные данные принадлежат пространствам $\Omega_{\infty}^{*}$ (определение $1.4, \S 2$, гл. 1 ) и $\Pi_{\infty}^{*}$ (определение $2.5, \S 2$, гл. 2), то ряды (3.3) и (3.4) абсолютно сходятся.

В связи с замечанием 3.2 представляется естественным сформулировать следующую гипотезу.

ГиПОтеЗА. Если при $|n| \rightarrow \infty$ модули функиий $\psi_{n}(\vec{q})$ и их производных достаточно быстро стремятся $\kappa$ нулю, то для начальных данных из пространств

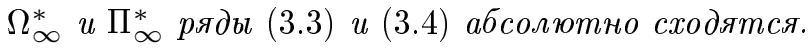

ОПРЕДЕЛЕНИЕ 3.2. Введем пространство $Y \subset \Omega^{*}$, состоящее из начальных данных, для которых при любых $t \in \mathbb{R}$ и $n \in \mathbb{Z}$ ряд (3.3) определяет целую аналитическую функцию по $t$, и пространство $X \subset \Pi^{*}$, состоящее из начальных данных, для которых при любых $t^{*} \in \mathbb{R}$ и $n \in \mathbb{Z}$ ряд (3.4) определяет целую аналитическую функцию по $t$.

ТЕОРема 3.3. Существуют единственное аналитическое решение $\left(q_{n}(t)\right)$ уравнения (3.1), определяемое начальнымм данными из пространства $Y$, и единственное аналитическое решение уравнения (3.2), определяемое начальными данным ми из пространства $X$.

Доказательство теоремы 3.3 проведем только для уравнения (3.1) и пространства $Y$, так как для уравнения (3.2) и пространства $X$ это осуществляется аналогичным образом. В силу определения 3.2 и теоремы 3.1 для начальных данных $\left\{\left(q_{n}^{*}\right),\left(v_{n}^{*}\right)\right\} \in$ 
$Y$ ряды (3.3) абсолютно сходятся и определяют формальное решение уравнения (3.1). Поэтому если при $n \in \mathbb{Z}$ мы рассмотрим аналитическую функцию

$$
f_{n}(t)=\varphi\left(q_{n}(t)\right)+q_{n+1}(t)-2 q_{n}(t)+q_{n-1}(t)+\psi_{n}(\vec{q}(t))-m_{n} \frac{d^{2} q_{n}}{d t^{2}}(t),
$$

то ее значение и значения всех ее производных в точке $t^{*}$ равны нулю, и, таким образом $f_{n}(t) \equiv 0$. Следовательно, аналитические функции (3.3) определяют решение $q_{n}(t)$ уравнения (3.1), которое в силу теоремы 3.1 будет единственньм.

Теорема 3.3 позволяет нам для целей следуюшего $\S 2$ ввести отображение $S$ пространств $Y$ и $X$ и расстояние $\rho(\cdot, \cdot)$ в них также, как это сделано в $\S 2$ в гл. 1 и в $\S 2$ в гл. 2.

А именно, если $\left\{\left(q_{n}^{*}\right),\left(v_{n}^{*}\right)\right\} \in Y$ и $\left\{\left(x_{n}^{*}\right),\left(v_{n}^{*}\right)\right\} \in X$, то полагаем

$$
S\left\{\left(q_{n}^{*}\right),\left(v_{n}^{*}\right)\right\}=\left(q_{0}(t), q_{1}(t)\right), \quad S\left\{\left(x_{n}^{*}\right),\left(v_{n}^{*}\right)\right\}=\left(x_{0}(t), x_{1}(t)\right),
$$

где $q_{0}(t), q_{1}(t)$ - аналитические функции, определенные равенствами (3.3), а $x_{0}(t)$, $x_{1}(t)$ - аналитические функции, определенные равенствами (3.4).

Если же рассмотреть две произвольные пары элементов $q^{\prime}=\left\{\left(q_{n}^{\prime}\right),\left(v_{n}^{\prime}\right)\right\} \in Y$, $q^{\prime \prime}=\left\{\left(q_{n}^{\prime \prime}\right),\left(v_{n}^{\prime \prime}\right)\right\} \in Y, x^{\prime}=\left\{\left(x_{n}^{\prime}\right),\left(v_{n}^{\prime}\right)\right\} \in X, x^{\prime \prime}=\left\{\left(x_{n}^{\prime \prime}\right),\left(v_{n}^{\prime \prime}\right)\right\} \in X$ в пространствах $Y$ и $X$, то можно определить расстояния

$$
\rho\left(q^{\prime}, q^{\prime \prime}\right)=\left\|S q^{\prime}-S q^{\prime \prime}\right\|^{(0)}, \quad \rho\left(x^{\prime}, x^{\prime \prime}\right)=\left\|S x^{\prime}-S x^{\prime \prime}\right\|^{(0)},
$$

где $\|\cdot\|^{(0)}$ - нормы, введенные в определении $1.5(\S 2$, гл. 1$)$ и в равенстве $(2.15)(\S 2$, гл. 2$)$ при $k=0$.

Для исследования проблемы устойчивости стационарных решений в $\S 2$ нам также потребуется следующее определение.

ОПРЕДЕЛЕНИЕ 3.3. Пусть $\sigma=\left(\sigma_{n}\right)$ - двусторонняя последовательность чисел $\sigma_{n} \geqslant 0(n \in \mathbb{Z})$.

Введем множества $Y^{(\sigma)}$ и $X^{(\sigma)}$ следуюшим образом: множество $Y^{(\sigma)}$ состоит из всех аналитических решений $\left(q_{n}(t)\right)$ уравнения (3.1),которые удовлетворяют неравенствам

$$
\sup _{t \in \mathbb{R}}\left|\frac{d^{2} q_{n}}{d t^{2}}(t)\right| \leqslant \sigma_{n}, \quad n \in \mathbb{Z}
$$

а множество $X^{(\sigma)}$ состоит из всех аналитических решений $\left(x_{n}(t)\right)$ уравнения $(3.2)$, которые удовлетворяют неравенствам

$$
\sup _{t \in \mathbb{R}}\left|\frac{d^{2} x_{n}}{d t^{2}}(t)\right| \leqslant \sigma_{n}, \quad n \in \mathbb{Z}
$$




\section{§ 2. Устойчивость стационарных решений}

В $\S 5,6$ гл. 1 была доказана устойчивость стационарного решения $\vec{q}^{0}=\left(q_{n}^{0}(t)\right)$, $q_{n}^{0} \equiv \eta+n a($ см. (1.25)) для уравнения (1.1), а в $\S \S 3,4$ гл. 2 была доказана устойчивость нулевого решения $\vec{x}^{0}=\left(x_{n}^{0}(t)\right), x_{n}^{0} \equiv 0$ для уравнения (2.1). В настоящем параграфе мы при некоторых обших предположениях устанавливаем более слабую устойчивость этих же решений для уравнений (3.1) и (3.2).

Применительно к уравнению (3.1) предположения заключаются в следующем:

1) $\psi_{n}\left(\vec{q}^{0}\right)=0$ при всех $n \in \mathbb{Z}$;

2) сушествуют такие числа $\theta>0, \tau$, что

$$
\sum_{n=-\infty}^{\infty} \sup _{\vec{q} \in \Gamma_{\theta}}\left|\psi_{n}(\vec{q})\right|<\tau,
$$

$$
\text { где } \Gamma_{\theta}=\left\{\vec{q}=\left(q_{n}\right):\left|q_{n}-q_{n}^{0}\right| \leqslant \theta, n \in \mathbb{Z}\right\} ;
$$

а применительно к уравнению (3.2) предположения имеют следующий вид:

$\left.1^{\prime}\right) \psi_{n}\left(\vec{x}^{0}\right)=0$ при всех $n \in \mathbb{Z}$;

$2^{\prime}$ ) существуют такие числа $\theta>0, \tau$, что

$$
\sum_{n=-\infty}^{\infty} \sup _{\vec{x} \in \Gamma_{\theta}^{\prime}}\left|\psi_{n}(\vec{x})\right|<\tau,
$$$$
\text { где } \Gamma_{\theta}^{\prime}=\left\{\vec{x}=\left(x_{n}\right):\left|x_{n}\right| \leqslant \theta, n \in \mathbb{Z}\right\} .
$$

ЗАмЕчАнИЕ 3.3. Условие 1) необходимо для того, чтобы вектор $\vec{q}^{0}$ был решением уравнения (3.1), условие $1^{\prime}$ ) необходимо для того, чтобы вектор $\vec{x}^{0}$ был решением уравнения (3.2).

Прежде, чем формулировать теоремы 3.4 и 3.5 , отметим, что стационарное решение $\vec{q}^{0}$ в пространстве $Y$ задается начальными данньми $\{(\eta+n a),(0)\}$, а нулевое решение $\vec{x}^{0}$ в пространстве $X$ задается начальными данными $\{(0),(0)\}$.

ТЕОРема 3.4. Предположим, что выполнены все условия теоремы 1.10 (§5, гл. 1) $и \sigma=\left(\sigma_{n}\right)$ - заранее заданная двусторонняя последовательность неотричательных чисел. Тогда для любого $\varepsilon>0$ найдутся числа $\delta>0, \tau_{0}>0$ и константы $m_{n}^{0}>0(n \in \mathbb{Z})$ такие, что если при всех $n \in \mathbb{Z} m_{n} \leqslant m_{n}^{0}$, неравенство (3.5) выполнено для $\tau \leqslant \tau_{0}$, а начальные данные $\left\{\left(q_{n}^{*}\right),\left(v_{n}^{*}\right)\right\} \in Y$ уравнения (3.1) удовлетворяют неравенству $\rho\left(\left\{\left(q_{n}^{*}\right),\left(v_{n}^{*}\right)\right\},\{(\eta+n a),(0)\}\right) \leqslant \delta$, то решение $\left(q_{n}(t)\right)$ уравнения (3.1) с этими начальными данными, принадлежсащее множеству $Y^{(\sigma)}$, при всех $n \in \mathbb{Z} u t \in \mathbb{R}$, удовлетворяет неравенству

$$
\left|q_{n}(t)-q_{n}^{0}\right| \leqslant \varepsilon
$$


Теорема 3.5. Предположим, что параметр с в уравнении (3.2) удовлетворяет неравенствам (2.23) в условии теоремы 2.4 (§3, гл. 2) $и \sigma=\left(\sigma_{n}\right)$ - заранее заданная двусторонняя последовательность неотрицательных чисел. Тогда для любого $\varepsilon>0$ найдутся числа $\delta>0, \tau_{0}>0$ и константы $m_{n}^{0}>0(n \in \mathbb{Z})$ такие, что если при всех $n \in \mathbb{Z} m_{n} \leqslant m_{n}^{0}$, неравенство (3.6) выполнено для $\tau \leqslant \tau_{0}$, а начальные данные $\left\{\left(x_{n}^{*}\right),\left(v_{n}^{*}\right)\right\} \in X$ уравнения (3.2) удовлетворяют неравенству $\rho\left(\left\{\left(x_{n}^{*}\right),\left(v_{n}^{*}\right)\right\},\{(0),(0)\}\right) \leqslant \delta$, то решение $\left(x_{n}(t)\right)$ уравнения $(3.2)$ с этими начальными данными, принадлежащее множеству $X^{(\sigma)}$, при всех $n \in \mathbb{Z} u t \in \mathbb{R}$, удовлетворяет неравенству

$$
\left|x_{n}(t)\right| \leqslant \varepsilon .
$$

Доказательства теорем 3.4 и 3.5 проводятся аналогично доказательствам теорем 1.10 и 2.4, сведением к теореме 2.6 (§ 5 , гл. 2). При этом в этих доказательствах роль преобразования $A$ из теоремы 2.6 играют те же преобразования, что и в доказательствах теорем 1.10 и 2.4 соответственно, а роль преобразований $B_{n}$ из теоремы 2.6 играют другие преобразования, которые отличаются от соответствуюших преобразований в доказательствах теорем 1.10 и 2.4 за счет членов, связанных с функциями $\psi_{n}$.

ЗАмЕчАниЕ 3.4. Устойчивость, доказанная в теоремах 3.4 и 3.5 , более слабая, чем в теоремах 1.10 и 1.24, так как она имеет место при изменении не только начальных данных, но и параметров.

ЗАмЕчАниЕ 3.5. Препятствием для получения результатов теорем 1.10 и 2.4 применительно к уравнениям (3.1) и (3.2) является необходимость иметь такую же явную реализацию фазовых пространств уравнений (3.1) и (3.2), какую мы имеем для уравнений (1.1) и (2.1) (теорема $1.5, \S 2$, гл. 1 ; теорема $2.3, \S 2$, гл. 2). Весьма вероятно, что такая реализация существует, и в этом случае стационарные решения $\vec{q}^{0}$ и $\vec{x}^{0}$ уравнений (3.1) и (3.2) будут устойчивы при изменении только начальных данных и фиксированных параметрах.

\section{СПИСОК ЛИТЕРАТУРЫ}

[1] Френкель Я.И., Конторова Т. А. О теории пластической деформации и двойникования // ЖЭТФ. 1938. Т. 8. С. 89-97.

[2] Fermi E., Pasta J., Ulam S. M. Studies of Nonlinear Problems. Washington, D.C.: LA-1940, Office of Technical Services, U.S. Dept. of Commerce..

[3] Улам C. Нерешенные математические задачи. М.: Наука, 1964.

[4] Fermi E., Pasta J.R., Ulam S. M. Studies of Nonlinear Problems // Collected Works of E. Fermi. V. 2. Chicago: Univ. Chicago Press, 1965.

[5] Колмогоров А. Н. О сохранении условно-периодических движений при малом изменении функции Гамильтона // Докл. АН СССР. 1954. Т. 98. № 4. С. 527-530.

[6] Арнольд В. И. Доказательство теоремы А.Н. Колмогорова о сохранении условно-периодических движений при малом изменении функции Гамильтона // УМН. 1963. Т. 18. №5. C. $13-40$.

[7] Арнольд В.И. Малые знаменатели и проблемы устойчивости движения в классической и небесной механике // УМН. 1963. Т. 18. №6. С. 91-192.

[8] Арнольд В.И. О неустойчивости динамической системы со многими степенями свободы // Докл. АН СССР. 1964. Т. 156. №1. С. 9-12.

[9] Arnold V.I. Stability problem and ergodic properties of classical dynamic systems // Proceedings of International Congress of Mathematicians. Moscow: Nauka, 1966. P. 387-392. 
[10] Arnold V. I. Mathematical Problem in Classical Physics // Trends and Perspectives in Applied Mathematics (Applied Mathematical Sciences 100) / ed. F. John, J.E. Marsden. Sirovich: Springer-Verlag, 1995.

[11] Арнольд В. И., Козлов В. В., Нейштадт А. И. Математические аспекты классической и небесной механики. Динамические системш 3 // Итоги науки и техники. Современные проблемы математики. Т. 3. М.: ВИНИТИ, 1985.

[12] Арнольд В. И. Математические методы классической механики. М.: Мир, 1974.

[13] Брюно А. Д. Множества аналитичности нормализующего преобразования // Препринт. № 97, 98. М.: АН СССР, Ин-т прикл. математики, 1974.

[14] Брюно А. Д. Ограниченная задача трех тел. М.: Наука, 1990.

[15] Мельников В. К. О некоторых случаях сохранения условно-периодических движений при малом изменении функции Гамильтона // Докл. АН СССР. 1965. Т. 165 . №6. C. $1245-1248$.

[16] Мельников В.К.О семействе условно-периодических решений гамильтоновой системы // Докл. АН СССР. 1968. Т. 81. № 3. С. 546-549.

[17] Мозер Ю. Об инвариантных кривых сохраняющего площадь отображения кольца в себя // Математика. 1963. Т. 6. № 5. С. 51-67.

[18] Moser J. Convergent series expansions for quasi-periodic motions // Math. Ann. 1967. V. 169. P. $136-176$

[19] Мозер Ю. Лекции о гамильтоновых системах. М.: Мир, 1973.

[20] Siegel C. L., Moser J. Lectures on Celestial Mechanics: Springer-Verlag, 1971.

[21] Zehnder E. Generalized implicit function theorem with applications to some small divisor problems, I, II // Comm. Pure Appl. Math. 1975. V. 28. P. 91-140;; 1976. V. 29. P. 49-111.

[22] Salamon D. Zehnder E. KAM theory in configuration space // Comment. Math. Helv. 1986. V. 64. P. 84-132.

[23] Нехорошев Н. Н. Экспоненциальная оценка времени устойчивости гамильтоновых систем, близких к интегрируемым // УМН. 1977. Т. 32. №6. С. 5-66.

[24] Frölich J., Spencer T., Wayne C. E. Localization in disordered nonlinear dynamical systems // J. Statist. Phys. 1986. V. 42. P. 247-274.

[25] Wayne C.E. Periodic and quasi-periodic solutions of nonlinear wave equations via KAM theory // Comm. Math. Phys. 1990. V. 127. P. 479-528.

[26] Wayne C.E. The KAM theory of systems with short range interactions. 1, 2 // Comm. Math. Phys. 1984. V. 96. P. 311-329; 331-344.

[27] Vittot M., Bellisard J. Invariant tori for an infinite lattice of coupled classical rotators // Preprint CPT. Marseille, 1985.

[28] Pöschel J. Small divisors with spatial structure in infinite dimensional Hamiltonian systems // Comm. Math. Phys. 1990. V. 127. P. 351-393.

[29] Kuksin S. B. Nearly integrable infinite-dimensional Hamiltonian systems // Lecture Notes in Math. V. 1556, 1993.

[30] Aubry S. The twist map, the extended Frenkel-Kontorova model and the Devil's Starcase // Physica D. 1983. V. 7. №1-3. P. 240-258.

[31] Aubry S., Le Daeron P. Y. The discret Frenkel-Kontorova model and its extensions // Physica D. 1983. V. 8. P. 381-422.

[32] Mather J. N. Existence of quasi-periodic orbits for twist homeomorphisms of the Annulus // Topology. 1982. V. 21. № 4. P. 457-467.

[33] Percival I. C. A variational principle for invariant tori of fixed frequency // J. Phys. A: Math. Nucl. Gen. 1979. V. 12. №3. P. 57-60.

[34] MacKay R. S., Percival I. C. Converse KAM: Theory and practice // Comm. Math. Phys. 1985. V. 98. P. 469-512.

[35] Sinai Ya. G. Topics in ergodic theory. Princeton NJ: Princeton Univ. Press, 1994.

[36] Sinai Ya. G. Commensurate-incommensurate phase transitions in one-dimensional chains // J. Statist. Phys. 1982. V. 29. P. 401-425.

[37] Синай Я. Г. Переходы “соизмеримость-несоизмеримость" в одномерных цепочках // ЖЭЭТ. 1982. Т. 83. C. 1223-1231. 
[38] Lazutkin V.F., Terman D. Percival variational principle for invariant measures and commensurate-incommensurate phase transitions in one-dimensional chains // Comm. Math. Phys. 1984. V. 94. № 4. P. 511-522.

[39] Заславский А.Я. Замечание о фазовой диаграмме в модели Френкеля-Конторовой // ТМФ. 1983. T. 57. C. 459-464.

[40] Pustyl'nikov L. D. On a grounde state in Frenkel-Kontorova model and metric properties of mappings of standart type // Adv. Soviet Math. 1994. V. 20. P. 277-289.

[41] Пустыльников Л. Д. Об одной динамической системе с бесконечным числом степеней свободы и о решении задачи Френкеля-Конторовой // ТМФ. 1986. Т. 68. № 1. С. 58-68.

[42] Пустыльников Л. Д. О построении периодических решений в бесконечной системе обыкновенных дифференциальных уравнений Ферми-Паста-Улама, об устойчивости и теории КАМ // УМН. 1995. Т. 50. № 2. С. 221-222.

[43] Пустыльников Л. Д. О неоднозначной разрешимости и о неразрешимости аналитического бесконечно-мерного обыкновенного дифференциального уравнения цепочечного типа // УМН. 1995. Т. 50. №6. С. 207-208.

[44] Пустыльников Л. Д. Модели Пуанкаре, строгое обоснование второго начала термодинамики из механики и механизм ускорения Ферми // УМН. 1995. Т. 50. № 1. С. 143-186.

[45] Пустыльников Л. Д. Неограниченный рост переменной действия в некоторых физических моделях // Труды ММО. 1983. Т. 46. С. 187-200.

[46] Pustyl'nikov L. D. On the stability of solutions and absence of Arnol'd diffusion in a nonintegrable Hamiltonian system of a general form with three degrees of freedom // Preprint. № 155. Berlin: Weierstraß-Institute für Angewandte Analysis und Stochastic, 1995.

[47] Пустыльников Л. Д. Об осцилирующих движениях в одной динамической системе // Изв. АН СССР. Сер. матем. 1987. Т. 51. № 5. С. 1010-1032.

[48] Пустыльников Л. Д. Бесконечно-мерные странные аттракторы и бифуркации в одной динамической системе с бесконечным числом степеней свободы // ТМФ. 1992. V. 92. №1. P. 85-91.

[49] Pustyl'nikov L. D. Many and infinite-dimensional Hamiltonian systems // Abstracts Inst. Math., Polish Acad. Sci.; Banach Center / ed. K. Baranski, F. Przytycki, 1995.

[50] Lokot' T. V., Pustyl'nikov L. D. On some solutions of infinite-dimensional systems of ordinary differential equations originating in statistical mechanics // Sinai's Moscow Seminar on Dinamical Systems. Adv. Math. Sci. AMS Transl. Ser. 2. V. 171, 1996. P. 173-183.

[51] Зигель К. Л. Лекции по небесной механике. М.: ИЛ, 1959.

[52] Картан Е. Интегральные инварианты. М.-Л.: Гостехиздат, 1940.

[53] Александров П. С. Введение в общую теорию множеств и функций. М: ГИТТЛ, 1948.

[54] Kuksin S. B. KAM - Theory for Partial Differential Equations // First European Congress of Mathematics. Vol. II, Invited Lectures (Part 2). Basel: Birkhäuser. P. 123-157.

[55] Пустыльников Л. Д. Теория уравнения с потенциалом Френкеля-Конторовой // Препринт. № 50. М.: РАН, Ин-т прикл. математики, 1996.

[56] Пустыльников Л. Д. Теория уравнения с нелинейностью Ферми-Паста-Улама // Препринт. № 55. М.: РАН, Ин-т прикл. математики, 1996.

[57] Пустыльников Л. Д. Бесконечномерные обькновенные дифференциальные уравнения общего вида // Препринт. № 84. М.: РАН, Ин-т прикл. математики, 1996. 